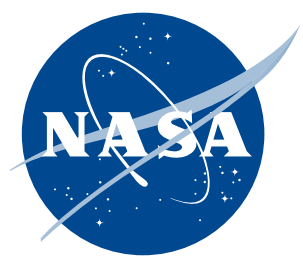

\title{
Numerical Determination of Critical Conditions for Thermal Ignition
}

W. Luo and G.C. Wake

Massey University, Auckland, New Zealand

C.W. Hawk

University of Alabama in Huntsville, Huntsville, Alabama

R.J. Litchford

Marshall Space Flight Center, Marshall Space Flight Center, Alabama 


\section{The NASA STI Program...in Profile}

Since its founding, NASA has been dedicated to the advancement of aeronautics and space science. The NASA Scientific and Technical Information (STI) Program Office plays a key part in helping NASA maintain this important role.

The NASA STI program operates under the auspices of the Agency Chief Information Officer. It collects, organizes, provides for archiving, and disseminates NASA's STI. The NASA STI program provides access to the NASA Aeronautics and Space Database and its public interface, the NASA Technical Report Server, thus providing one of the largest collections of aeronautical and space science STI in the world. Results are published in both nonNASA channels and by NASA in the NASA STI Report Series, which includes the following report types:

- TECHNICAL PUBLICATION. Reports of completed research or a major significant phase of research that present the results of NASA programs and include extensive data or theoretical analysis. Includes compilations of significant scientific and technical data and information deemed to be of continuing reference value. NASA's counterpart of peerreviewed formal professional papers but has less stringent limitations on manuscript length and extent of graphic presentations.

- TECHNICAL MEMORANDUM. Scientific and technical findings that are preliminary or of specialized interest, e.g., quick release reports, working papers, and bibliographies that contain minimal annotation. Does not contain extensive analysis.

- CONTRACTOR REPORT. Scientific and technical findings by NASA-sponsored contractors and grantees.
- CONFERENCE PUBLICATION. Collected papers from scientific and technical conferences, symposia, seminars, or other meetings sponsored or cosponsored by NASA.

- SPECIAL PUBLICATION. Scientific, technical, or historical information from NASA programs, projects, and missions, often concerned with subjects having substantial public interest.

- TECHNICAL TRANSLATION. Englishlanguage translations of foreign scientific and technical material pertinent to NASA's mission.

Specialized services also include creating custom thesauri, building customized databases, and organizing and publishing research results.

For more information about the NASA STI program, see the following:

- Access the NASA STI program home page at $<$ http://www.sti.nasa.gov>

- E-mail your question via the Internet to $<$ help@sti.nasa.gov>

- Fax your question to the NASA STI Help Desk at 301-621-0134

- Phone the NASA STI Help Desk at 301-621-0390

- Write to: NASA STI Help Desk NASA Center for AeroSpace Information 7115 Standard Drive Hanover, MD 21076-1320 
NASA/TP-2008-215194

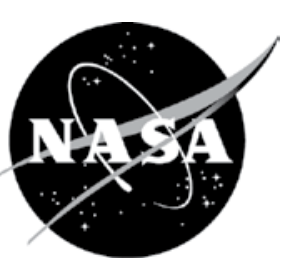

\section{Numerical Determination of Critical Conditions for Thermal Ignition}

W. Luo and G.C. Wake

Massey University, Auckland, New Zealand

C.W. Hawk

University of Alabama in Huntsville, Huntsville, Alabama

R.J. Litchford

Marshall Space Flight Center, Marshall Space Flight Center, Alabama

National Aeronautics and

Space Administration

Marshall Space Flight Center • MSFC, Alabama 35812 
Available from:

NASA Center for AeroSpace Information

7115 Standard Drive

Hanover, MD 21076-1320

301-621-0390

This report is also available in electronic form at $<$ https://www2.sti.nasa.gov> 


\section{TABLE OF CONTENTS}

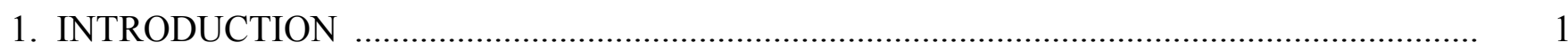

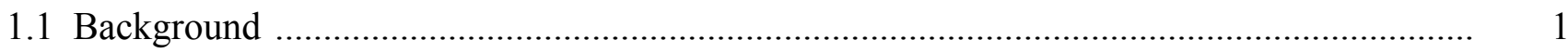

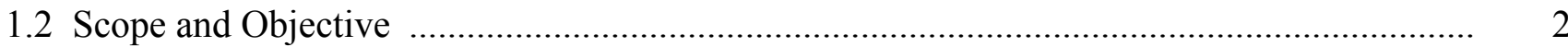

1.3 Dimensionless Formulations of the Reaction-Diffusion Equation ......................................... 3

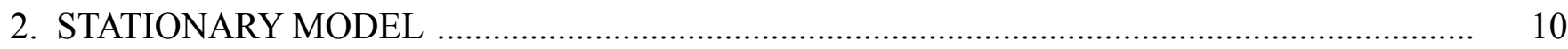

2.1 Qualitative Structure of Solution Branches …………...................................................... $\quad 10$

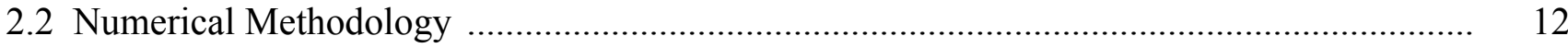

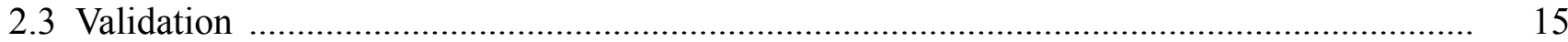

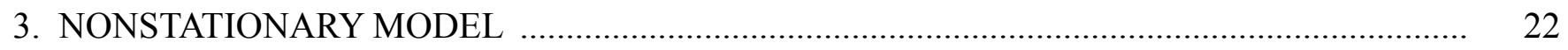

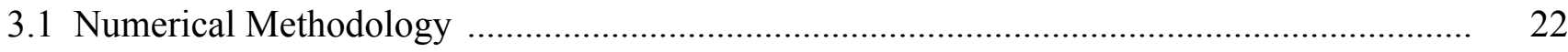

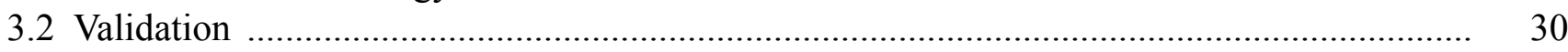

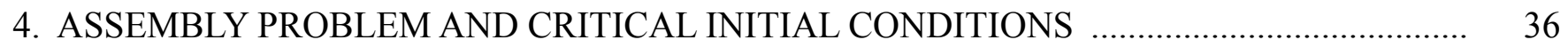

4.1 Initial Shape Profile ................................................................................................. 36

4.2 Criticality in Nonuniform Assemblies ........................................................................... 37

5. CORRELATION AND REDUCTION OF CRITICAL ASSEMBLY CONDITIONS .................. 51

5.1 Correlating Forms and Structural Compaction ………………………................................ 51

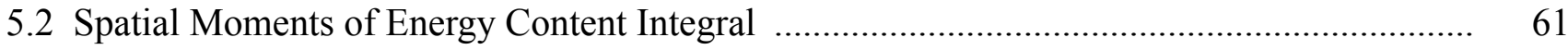

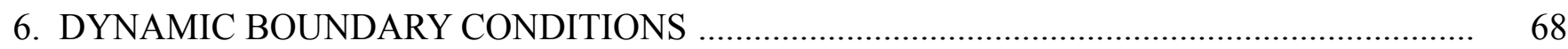

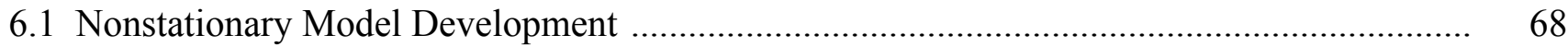

6.2 Parametric Survey .................................................................................................. $\quad 71$

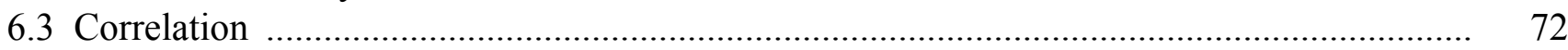

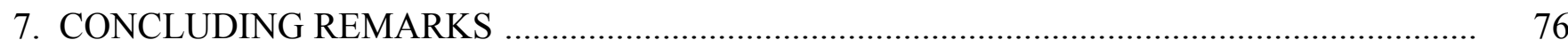

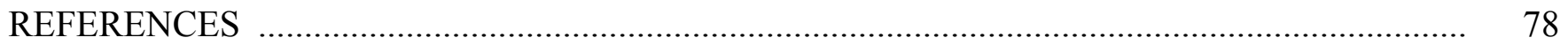




\section{LIST OF FIGURES}

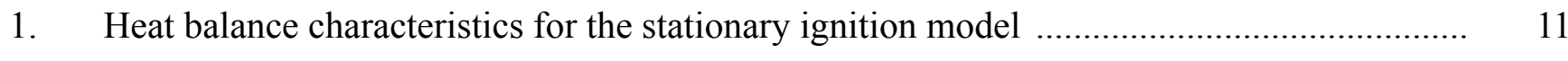

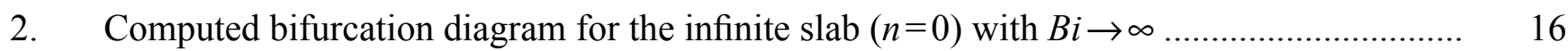

3. Computed bifurcation diagram for the infinite cylinder $(n=1)$ with $B i \rightarrow \infty \ldots \ldots \ldots \ldots \ldots \ldots \ldots \ldots . . . . . . . . . . . . . .17$

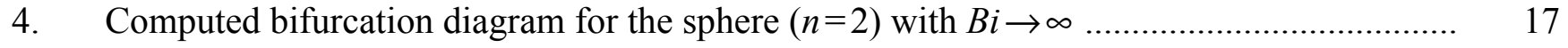

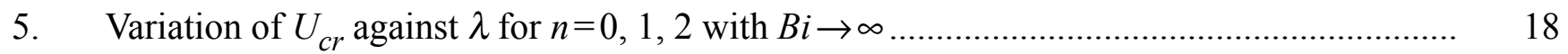

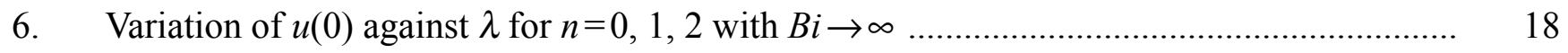

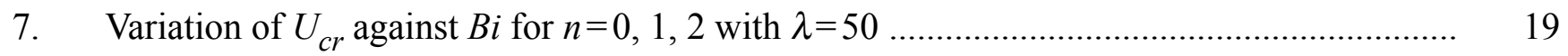

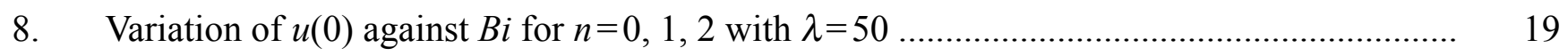

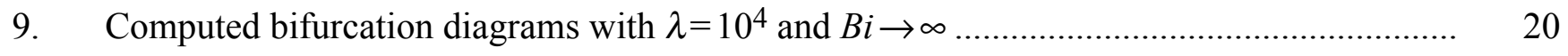

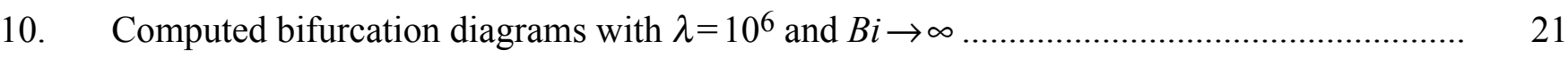

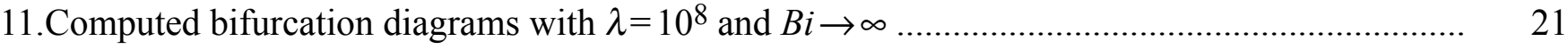

12. Computational grid structure at external boundary including fictitious node ........................ 25

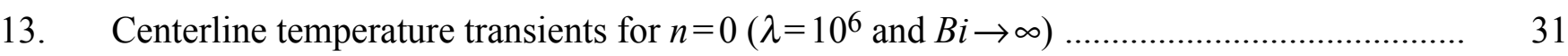

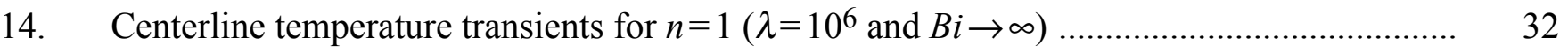

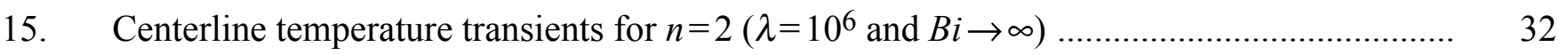

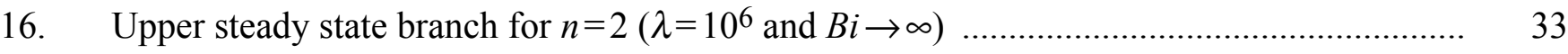

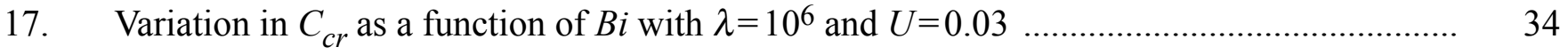

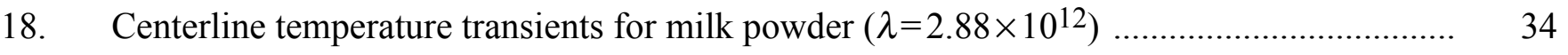

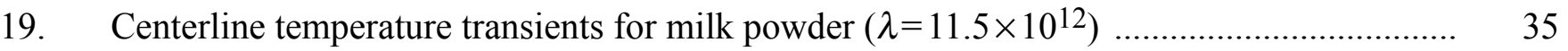

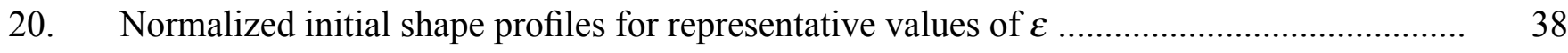




\section{LIST OF FIGURES (Continued)}

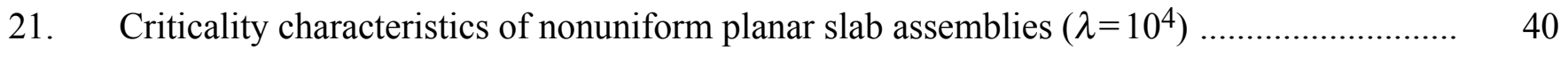

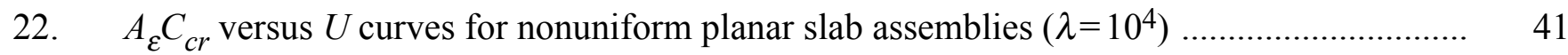

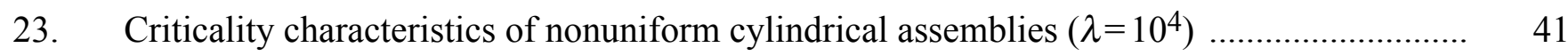

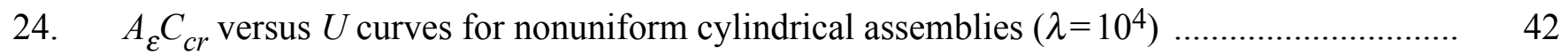

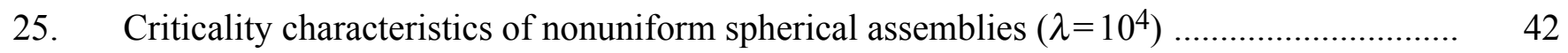

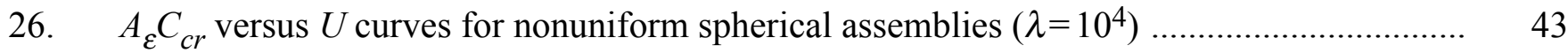

27. Criticality characteristics of nonuniform planar slab assemblies $\left(\lambda=10^{6}\right) \ldots \ldots \ldots \ldots \ldots \ldots \ldots \ldots \ldots \ldots . . . . . . . . . . . . .43$

28. $\quad A_{\varepsilon} C_{c r}$ versus $U$ curves for nonuniform planar slab assemblies $\left(\lambda=10^{6}\right) \ldots \ldots \ldots \ldots \ldots \ldots \ldots \ldots \ldots \ldots \ldots . . . . . . . . \ldots \ldots$

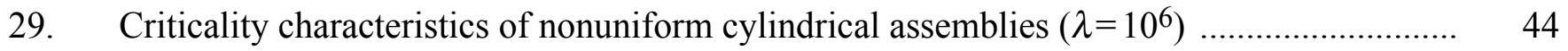

30. $A_{\varepsilon} C_{c r}$ versus $U$ curves for nonuniform cylindrical assemblies $\left(\lambda=10^{6}\right) \ldots \ldots \ldots \ldots \ldots \ldots \ldots \ldots \ldots \ldots \ldots . . . . . . . . . . . . . .45$

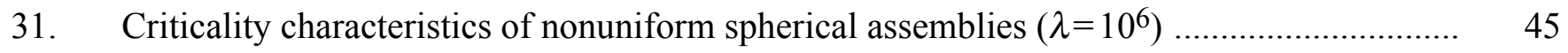

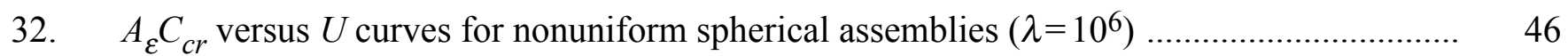

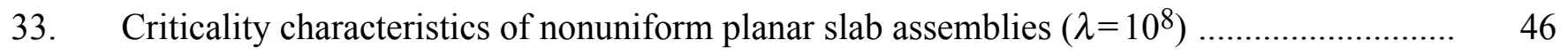

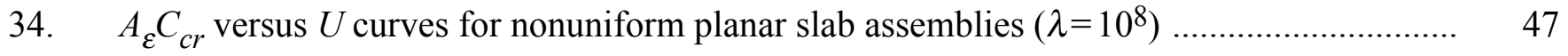

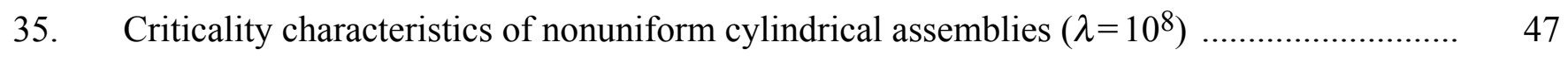

36. $A_{\varepsilon} C_{c r}$ versus $U$ curves for nonuniform cylindrical assemblies $\left(\lambda=10^{8}\right) \ldots \ldots \ldots \ldots \ldots \ldots \ldots \ldots \ldots \ldots \ldots . . . . . . . . . . . . . .48$

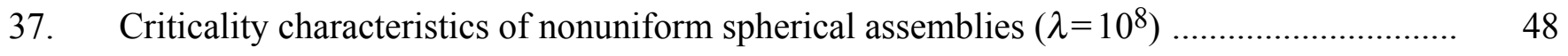

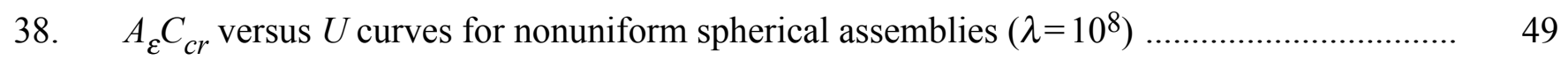

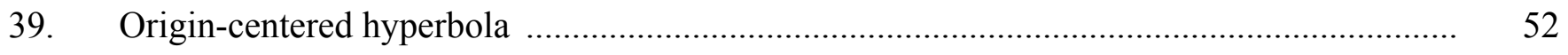

40. $\quad A_{\varepsilon} C_{c r}$ versus $U$ results for nonuniform planar slab assemblies $\left(\lambda=10^{4}\right)$.

Precise computational value — symbols. Hyperbolic correlation—curves …………….................... 54 


\section{LIST OF FIGURES (Continued)}

41. $\quad A_{\varepsilon} C_{c r}$ versus $U$ results for nonuniform planar slab assemblies $\left(\lambda=10^{6}\right)$.

Precise computational value-symbols. Hyperbolic correlation-curves

42. $A_{\varepsilon} C_{c r}$ versus $U$ results for nonuniform planar slab assemblies $\left(\lambda=10^{8}\right)$.

Precise computational value - symbols. Hyperbolic correlation - curves

43. $\quad A_{\varepsilon} C_{c r}$ versus $U$ results for nonuniform cylindrical assemblies $\left(\lambda=10^{4}\right)$.

Precise computational value - symbols. Hyperbolic correlation — curves

44. $A_{\varepsilon} C_{c r}$ versus $U$ results for nonuniform cylindrical assemblies $\left(\lambda=10^{6}\right)$.

Precise computational value - symbols. Hyperbolic correlation — curves

45. $\quad A_{\varepsilon} C_{c r}$ versus $U$ results for nonuniform cylindrical assemblies $\left(\lambda=10^{8}\right)$.

Precise computational value - symbols. Hyperbolic correlation — curves

46. $\quad A_{\varepsilon} C_{c r}$ versus $U$ results for nonuniform spherical assemblies $\left(\lambda=10^{4}\right)$.

Precise computational value - symbols. Hyperbolic correlation — curves

47. $\quad A_{\varepsilon} C_{c r}$ versus $U$ results for nonuniform spherical assemblies $\left(\lambda=10^{6}\right)$.

Precise computational value - symbols. Hyperbolic correlation - curves

48. $\quad A_{\varepsilon} C_{c r}$ versus $U$ results for nonuniform spherical assemblies $\left(\lambda=10^{8}\right)$.

Precise computational value - symbols. Hyperbolic correlation — curves

49. Universal correlating line. Slab $(n=0)$

50. Universal correlating line. Cylinder $(n=1)$

51. Universal correlating line. Spherical $(n=2)$

52. $\psi_{n, m}$ versus $U / U_{c r}$ for nonuniform slab assemblies $(n=0, m=1)$

53. $\psi_{n, m}$ versus $U / U_{c r}$ for nonuniform slab assemblies $(n=0, m=2)$

54. $\psi_{n, m}$ versus $U / U_{c r}$ for nonuniform cylindrical assemblies $(n=1, m=1) \ldots$

55. $\psi_{n, m}$ versus $U / U_{c r}$ for nonuniform cylindrical assemblies $(n=1, m=2)$

56. $\psi_{n, m}$ versus $U / U_{c r}$ for nonuniform spherical assemblies $(n=2, m=1)$ 66

57. $\psi_{n, m}$ versus $U / U_{c r}$ for nonuniform spherical assemblies $(n=2, m=2)$ 


\section{LIST OF FIGURES (Continued)}

58. Arithmetically averaged values of spatial moment integrals, $\bar{\psi}_{n, m}$

59. Centerline temperature transients for $n=0\left(\lambda=10^{6}\right.$ and $\left.B i \rightarrow \infty\right)$ with sinusoidal oscillating ambient temperature $\left(\bar{U}=0.05, U^{\prime}=0.001\right.$, and $\left.T=0.1\right)$

60. Centerline temperature transients for $n=2\left(\lambda=10^{6}\right.$ and $\left.B i \rightarrow \infty\right)$ with sinusoidal oscillating ambient temperature $\left(\bar{U}=0.05, U^{\prime}=0.001\right.$, and $\left.T=0.1\right)$

61. Variation in $\tau_{\text {ind }}$ as a function of $U$ for $n=0\left(\lambda=10^{6}\right.$ and $\left.B i \rightarrow \infty\right)$

62. Criticality characteristics $\left(n=0, \lambda=10^{6}, \bar{U}=0.05\right.$, and $\left.B i \rightarrow \infty\right)$ with sinusoidal oscillating ambient temperature $(T=0.05)$

63. Criticality characteristics $\left(n=0, \lambda=10^{6}, \bar{U}=0.05\right.$, and $\left.B i \rightarrow \infty\right)$ with sinusoidal oscillating ambient temperature $(T=0.05)$

64. Variation in $U_{c r}$ with $U^{\prime}$ and $T$ for $n=0\left(\lambda=10^{6}\right.$ and $\left.B i \rightarrow \infty\right)$ with sinusoidal oscillating ambient temperature

65. Stationary model correlation for $B i_{\text {eff }}$ as a function of $U^{\prime}$ and $T$ for $n=0$ $\left(\lambda=10^{6}\right.$ and $\left.B i \rightarrow \infty\right)$ with sinusoidal oscillating ambient temperature 



\section{LIST OF TABLES}

1. Mathematical relationship between alternative dimensionless variables ……....................... 8

2. $\quad$ Values of $R_{s}, R_{0}$, and $n$ for some common geometries .................................................. 9

3. Relationship between weighting factor and integration scheme ....................................... 24

4. Summary of profile shape factors and normalization factors …………............................. 38

5. Optimal fitting parameters for hyperbolic correlation ………….................................... 54

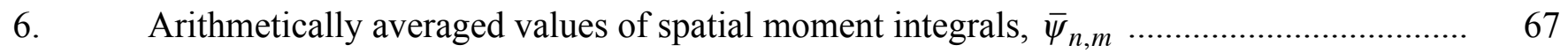

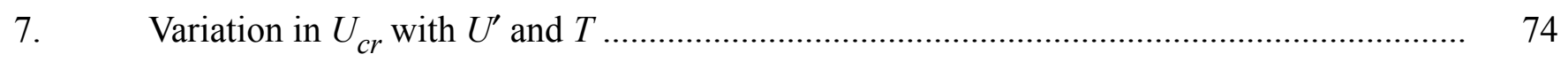

\section{LIST OF ACRONYMS}

TDMA Tri-Diagonal Matrix Algorithm 


\title{
NOMENCLATURE
}

\author{
A pre-exponential factor in the Arrhenius reaction rate term \\ $A_{\varepsilon} \quad$ normalization factor for preserving total heat content irrespective of profile shape \\ a radius from $O$ to a point on the surface \\ Bi dimensionless Biot number \\ $B i_{\text {eff }} \quad$ an effective Biot number \\ C arbitrary constant representing an initial perturbative displacement from thermal equilibrium \\ $C_{c r} \quad$ critical value for $C$ \\ $c_{p} \quad$ specific heat \\ $d \omega \quad$ solid angle subtended at the center $O$ \\ E total energy content \\ $E_{a} \quad$ activation energy \\ $\hat{E} \quad$ dimensionless total energy content \\ e energy density \\ $g(\xi) \quad$ a one-parameter shape profile to define a generalized nonuniform initial \\ temperature distribution \\ $H(T) \quad$ rate of heat production per unit volume at temperature $T$ \\ $H(u) \quad$ Heaviside function, as a multiplicative factor to the source term \\ $h \quad$ convective heat transfer coefficient \\ $K U \quad K, L$, and $M$ are $N \times N$ square matrices containing numerical coefficients and $U$ is an \\ $\mathrm{N}$-element column vector containing the dependent variable values at the grid points \\ $K^{-1} \quad$ matrix inverse of $K$
}




\section{NOMENCLATURE (Continued)}

$k \quad$ thermal conductivity

$L \quad$ characteristic length; represents the half-width of a symmetrical bounded region

$L U \quad K, L$, and $M$ are $N \times N$ square matrices containing numerical coefficients and $U$ is an $N$-element column vector containing the dependent variable values at the grid points

$M \quad K, L$, and $M$ are $N \times N$ square matrices containing numerical coefficients and $U$ is an $\mathrm{N}$-element column vector containing the dependent variable values at the grid points

$m \quad$ initial time step

m- $\quad$ spatial moment

$N \quad$ surface boundary node

$n \quad$ serves as a geometry selection parameter

$Q \quad$ heat of reaction per unit mass (i.e., "exothermicity”)

$R \quad$ universal gas constant

$R_{0} \quad$ harmonic mean radius

$R_{S} \quad$ Semenov radius

$S \quad$ surface area

$S \quad$ physical spatial coordinate referenced to the axis of symmetry

T temperature

$t \quad$ the time

$T_{a} \quad$ ambient temperature of the surrounding environment

$T_{S} \quad$ material surface temperature

$U \quad$ dimensionless ambient temperature

$U^{\prime} \quad$ fluctuation amplitude 


\section{NOMENCLATURE (Continued)}

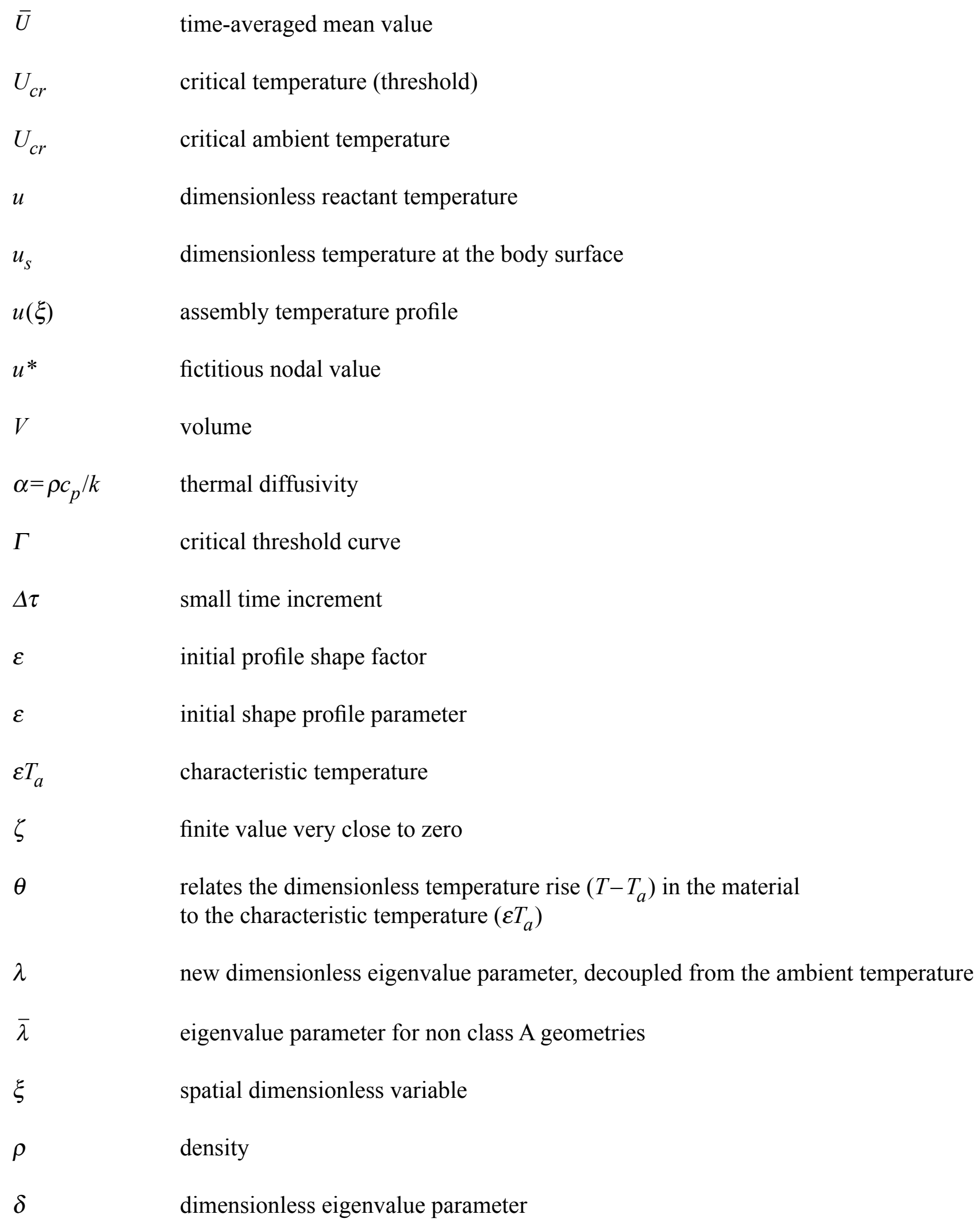


NOMENCLATURE (Continued)

$\begin{array}{ll}\delta \Omega & \text { on the smooth surface } \\ \tau & \text { time dimensionless variable?? } \\ \varphi & \text { phase angle } \\ \bar{\psi}_{n, m} & \text { spatial moments integrals } \\ \Omega & \text { bounded region }\end{array}$



TECHNICAL PUBLICATION

\section{NUMERICAL DETERMINATION OF CRITICAL CONDITIONS FOR THERMAL IGNITION}

\section{INTRODUCTION}

\subsection{Background}

Ignition or thermal explosion of a combustible substance occurs when exothermic reactions evolve heat so rapidly that it is impossible to preserve a stable balance between heat production and the loss of heat to the surroundings. This is an essential feature of many technological devices that employ auxiliary heat sources as a means of accelerating internal heat generation and engendering a runaway increase in temperature. On the other hand, there are numerous industrial processes involving the production and storage of reactive materials in which self-heating effects can culminate in spontaneous combustion or explosive effects and the primary concern is avoiding the occurrence of potentially hazardous circumstances. ${ }^{1}$

The archetypal example of self-heating is a porous pile of material in which heat is internally generated by atmospheric oxidation. If the excess heat in the pile can be transported and dissipated to the surroundings fast enough, an equilibrium or steady state can be safely established. Under certain conditions, however, the dissipation mechanism cannot keep pace with the self-heating rate, and spontaneous ignition or explosion will occur. These critical conditions depend on the size and shape of the pile, the assembly temperature of the material, and the ambient temperature of the surrounding environment.

Historically, assessment and control of self-heating hazards have been mainly conducted on an empirical basis, as established through long years of experience in the handling and processing of susceptible materials and products. The contemporary trend, however, is towards the development of reliable mathematical models and quantitative predictive procedures. In fact, the basic theoretical construct may be erected in a rather straightforward manner using conservation principles and well established descriptions of the underlying chemical and physical processes. The resulting mathematical formulation is commonly referred to as the reaction-diffusion equation:

$$
\rho c_{p} \frac{\partial T}{\partial t}=\nabla(k \nabla T)+H(T),
$$

where $t$ is the time, $T$ is the temperature, $\rho$ is the density, $c_{p}$ is the specific heat, $k$ is the thermal conductivity, and $H(T)$ is the rate of heat production per unit volume at temperature $T$. This nonlinear partial differential equation is a localized expression of the conservation of energy and implies that the rate of change of thermal energy within a unit volume element is equal to the net conduction heat transfer through the bounding surface plus the volumetric heat generation rate. 
For most ignition problems of practical importance, it is possible to incorporate certain simplifying assumptions which make the mathematics more tractable. These include the following:

- Negligible reactant consumption and reactant diffusion (i.e., "zero order" reaction).

- Constant thermal conductivity.

- Arrhenius temperature dependence for the exothermic reaction rate.

Enforcement of these assumptions yields the following working form for the reaction-diffusion equation:

$$
\alpha \frac{\partial T}{\partial t}=\nabla^{2} T+\frac{\rho Q A}{k} e^{-E_{a} / R T}
$$

where $\alpha=\rho c_{p} / k$ is the thermal diffusivity, $Q$ is the heat of reaction per unit mass (i.e., 'exothermicity'), $A$ is the pre-exponential factor in the Arrhenius reaction rate term, $E_{a}$ is the activation energy, and $R$ is the universal gas constant. When applied over a bounded region $\Omega$, energy conservation principles yield a generalized boundary condition on the smooth surface $\delta \Omega$

$$
k \frac{\partial T}{\partial n}=h\left(T_{a}-T_{s}\right)
$$

where $\partial / \partial n$ is the outward normal derivative on $\partial \Omega, h$ is the convective heat transfer coefficient, $T_{a}$ is the ambient temperature of the surrounding environment, and $T_{S}$ is the material surface temperature.

Given the shape and size of a bounded region, appropriate boundary conditions, and values for the fundamental material properties, the basic objective is to mathematically exploit the reaction-diffusion equation and determine the critical parameters and conditions leading to the onset of ignition or thermal explosion. In particular, we are concerned with predictions for the critical ambient temperature, which defines an external environmental constraint for safe 'storage,' and the critical initial temperature, which defines an internal constraint for safe 'assembly.'

\subsection{Scope and Objective}

From a mathematical perspective, there are two fundamental strategies for attacking the reaction-diffusion equation and determining critical conditions for thermal ignition. These are the stationary (steady-state) model and the nonstationary (transient) model.

In the stationary model, the time-dependent term in equation (2) is neglected and steady-state solutions are sought for which heat losses exactly balance heat production. This approach assumes unlimited reactants and implies that either a small steady-state excess temperature will become established in the body or the temperature will increase indefinitely. The principal attraction of the stationary modeling approach is a reduction of the problem to more amenable ordinary differential equation form, which has facilitated the development and refinement of standard mathematical methods capable of accounting for internal spatial temperature distributions and producing reliable estimates for the critical ambient temperature. Because the stationary model cannot account for time evolution, however, it has only limited effectiveness in the prediction of critical initial conditions. It is well known, for instance, that many fires have 
resulted from the assembly of reactive material at too high an initial temperature even though the storage conditions were sub-critical on the basis of steady state theory. See, for example, Bowes ${ }^{1}$ (who refers to this as thermal explosion of the second kind), Rivers et al., ${ }^{2}$ and Smedley and Wake. ${ }^{3}$

The nonstationary model, on the other hand, retains the complete time-dependent form of the reaction-diffusion equation and evolves the full temperature history of the self-heating body. The drawback of this approach is the general need to resort to numerical analysis and the fact that a full development history must be computed for each initial/boundary condition of potential interest. The distinct advantage, however, is that it can fully account for the initial assembly conditions and is therefore able to provide accurate estimates for the critical initial temperature. Weber et al., ${ }^{4}$ for instance, recently conducted a limited computational study of the nonstationary model which clearly demonstrated that the practical critical assembly temperature may, under certain circumstances, be 5-10\% lower than the critical temperature obtained from steady-state theory. This has profound industrial implications in the assessment of fire hazards and the definition of fire safety standards, for which a clear distinction must be drawn between the classic 'storage' problem, where only steady-state temperatures are important, and the less recognized 'assembly' problem, where the initial temperature threshold for self-ignition is of vital concern.

Physical situations involving dynamical boundary conditions, where the ambient temperature or surface heat flux has a known time-dependent variation, represent an additional class of problems that require a nonstationary mathematical treatment for the accurate prediction of critical ignition conditions. Such 'dynamic regimes of ignition' might include the behavior of a combustible material under the action of an igniter with a predefined heat deposition rate or a reactive industrial stockpile exposed to diurnal variations in ambient temperature. Clearly, the standard stationary model results for 'static regimes of ignition' are inapplicable to this class of problem, and nonstationary approaches are the only viable course of action at the present time.

The central objective of this research is the development and use of numerical techniques (1) to investigate the nonstationary solution sets of the full time-dependent reaction-diffusion equation subject to a general convective boundary condition and (2) to determine the critical threshold that distinguishes between initial conditions that evolve to a low-temperature steady-state and those that evolve to a hightemperature steady-state attractor (i.e., thermally ignite). This numerical methodology is then used as the basis for the following three-pronged research program: (1) conduct a broad ranging numerical study of the 'assembly' problem using a generalized one-parameter power law for the initial temperature profile; (2) investigate the relationship between the shape of the critical initial temperature distribution and the corresponding spatial moments of its energy content integral and attempt to forge a fundamental conjecture governing this relation; and (3) investigate the effect of dynamic boundary conditions on the classic 'storage' problem and use the results of the nonstationary model to lay the groundwork for the development of an approximate solution methodology based on adaptation of the standard stationary model.

\subsection{Dimensionless Formulations of the Reaction-Diffusion Equation}

Evaluation and analysis of the reaction-diffusion equation is facilitated by the introduction of dimensionless parameters. From a historical perspective, it is important to note the traditional grouping of dimensionless variables suggested by Frank-Kamenetskii in the first comprehensive analytical treatment of the stationary model. ${ }^{5}$ This classical formulation has been highly influential over the years, since 
it facilitates certain simplifying approximations in the nonlinear Arrhenius rate term, making it amenable to analytical attack. In fact, virtually all subsequent theoretical developments have utilized this standard form as a common starting point. From a modern perspective, however, an alternative formulation based on the Burnell-GrahamEagle-Gray-Wake variables provides less circuitous contact with the primitive variables and enables a more direct physical interpretation. ${ }^{6}$ Moreover, the facilitating features of the Frank-Kamenetskii variables are not necessarily advantageous for comprehensive numerical analyses where there is less need for simplifying assumptions. Thus, the mathematical treatment in this work will be constructed exclusively on the modern variable formulation. For the sake of completeness, however, and to facilitate translation and comparison between the two frames of reference, both formulations are briefly outlined in the following subsections.

\subsubsection{Frank-Kamenetskii Variables}

The Frank-Kamenetskii grouping of dimensionless variables was originally introduced as a means of simplifying the temperature dependence of the Arrhenius reaction rate term to permit the construction of exact analytical solutions. Because these variables appeared in the pioneering theoretical developments of the field, however, they became a de facto standard and tended to permeate all subsequent developments, despite certain drawbacks in clarity and interpretation. Thus, basic knowledge of the Frank-Kamenetskii formulation is essential as a frame of reference for understanding previous theoretical work.

Development of this formulation begins with the definition of parameters for the dimensionless ambient temperature and the dimensionless reactant temperature,

$$
\varepsilon=\frac{R T_{a}}{E_{a}} \quad \text { and } \quad \theta=\frac{T-T_{a}}{\varepsilon T_{a}} .
$$

Note that both parameters include the ambient temperature as a scaling factor and are therefore coupled. Thus, $\theta$ relates the dimensionless temperature rise $\left(T-T_{a}\right)$ in the material to the characteristic temperature $\left(\varepsilon T_{a}\right)$. From the above definitions, one may readily establish the identity

$$
-\frac{E_{a}}{R T} \equiv-\frac{1}{\varepsilon}+\frac{\theta}{1+\varepsilon \theta},
$$

which leads to a dimensionless expression for the temperature dependence of the Arrhenius reaction rate term, $e^{-1 / \varepsilon} e^{\theta /(1+\varepsilon \theta)}$. Because $\varepsilon^{-1}=E_{a} / R T_{a}$ is in the range of 10-100 for most materials, the nonlinearity $\exp [\theta /(1+\varepsilon \theta)]$ is convex for small positive $\theta$ and concave for $\theta>\left(\varepsilon-\varepsilon^{2}\right) / 2$.

We next rescale and normalize the spatial and time coordinates by defining the following dimensionless variables:

$$
\xi=\frac{s}{L} \quad \text { and } \quad \tau=\frac{t}{\alpha L^{2}},
$$

where the characteristic length $L$ represents the half-width of a symmetrical bounded region and $s$ is the physical spatial coordinate referenced to the axis of symmetry. Substitution of the above dimensionless 
variables into the reaction-diffusion equations (2) and (3) yields the Frank-Kamenetskii formulation in conventional compact form:

$$
\frac{\partial \theta}{\partial \tau}=\nabla_{\xi}^{2} \theta+\delta e^{\theta /(1+\varepsilon \theta)} \quad \text { in } \Omega
$$

and

$$
\frac{\partial \theta}{\partial \xi}+B i \theta=0 \quad \text { on } \partial \Omega .
$$

Here, $B i=h L / k$ is the dimensionless Biot number and $\delta$ is a dimensionless eigenvalue parameter given by

$$
\delta=\frac{E_{a}}{R T_{a}^{2}} \frac{L^{2} \rho Q A}{k} e^{-E_{a} / R T_{a}}
$$

It is convenient at the outset to develop a generalized formulation that is applicable to all three principal centrosymmetric solids, commonly referred to as the Class A geometries; i.e., the slab, infinite cylinder, and sphere. This is accomplished by expanding the Laplacian operator in cartesian, cylindrical, and spherical coordinates and observing that these can all be represented in the parameterized form:

$$
\nabla_{\xi}^{2} \theta=\frac{\partial^{2} \theta}{\partial \xi^{2}}+\frac{n}{\xi} \frac{\partial \theta}{\partial \xi},
$$

where $n$ serves as a geometry selection parameter. That is, $n=0,1$, or 2 for the slab, infinite cylinder, and sphere, respectively. Hence, the final working form of the reaction-diffusion equation for class A shapes may be written as

$$
\frac{\partial \theta}{\partial \tau}=\frac{\partial^{2} \theta}{\partial \xi^{2}}+\frac{n}{\xi} \frac{\partial \theta}{\partial \xi}+\delta e^{\theta /(1+\varepsilon \theta)} \quad \text { in } \Omega
$$

and

$$
\frac{\partial \theta}{\partial \xi}+B i \theta=0 \quad \text { on } \partial \Omega
$$

The Frank-Kamenetskii arrangement of the reaction-diffusion equation, in the form above, contains no additional simplifying assumptions and is appropriate for rigorous analyses of stationary and nonstationary models of thermal ignition. In fact, it has been the commonly used formulation in almost all previous mathematical and computational studies.

Exact analytical attacks on the stationary model, however, require further simplifications to the nonlinear Arrhenius reaction rate term, and it is of passing historical interest to note the classic FrankKamenetskii approximation valid when $\varepsilon<<1$ and $\theta$ is not large. Under this assumption, equation (5) takes the simpler form

$$
-\frac{E_{a}}{R T} \approx-\varepsilon^{-1}+\theta
$$


One should note that the distinct advantage of the Frank-Kamenetskii variables - indeed the fundamental reason for their original introduction - is the simplification provided by this approximation. Other simplified representations of the Arrhenius term, such as the quadratic approximation of Boddington et al., ${ }^{7}$ have been exploited for the development of exact analytical solutions in the Frank-Kamenetskii variables.

Therefore, these variables have been particularly useful in the construction of analytical solutions for the stationary model, where we are interested in the behavior of $\theta$ under variations in the eigenvalue parameter $\delta$. In carrying out the exact analysis, one finds that there are two solution branches for $\theta$ when $\delta<\delta_{c r}$ : the lower branch being stable and the upper branch being unstable. When $\delta>\delta_{c r}$, however, no solutions exist. Thus, $\delta$ may be considered as a bifurcation parameter in the Frank-Kamenetskii formulation such that $\delta_{c r}$ represents the first limit point of the corresponding bifurcation diagram. From a physical perspective, $\delta_{c r}$ is identified with the onset of ignition, since for $\delta>\delta_{c r}$ no steady-state solution exists and the temperature of the body will rise in time without bound.

\subsubsection{Burnell-GrahamEagle-Gray-Wake Variables}

Despite its predominance in the literature, the traditional Frank-Kamenetskii grouping of dimensionless variables has the effect of confusing the role of the ambient temperature when, in fact, it is the most practically significant control parameter in the problem definition. To circumvent this difficulty, Burnell et al. suggested an alternative dimensionless grouping with temperature rescaled independently of the ambient temperature. ${ }^{6}$

The major distinction in the new grouping is the definition of a dimensionless reactant temperature and a dimensionless ambient temperature that are completely decoupled:

$$
u=\frac{R T}{E_{a}} \quad \text { and } \quad U=\frac{R T_{a}}{E_{a}} .
$$

As in the Frank-Kamenetskii formulation, we rescale and normalize the spatial and time coordinates using the previously defined dimensionless variables $\xi=s / L$ and $\tau=t / \alpha L^{2}$. Then, substitution of the dimensionless parameters into the reaction-diffusion equations (2) and (3) yields the BurnellGrahamEagle-Gray-Wake formulation in compact form:

$$
\frac{\partial u}{\partial \tau}=\nabla_{\xi}^{2} u+\lambda e^{-1 / u} \quad \text { in } \Omega
$$

and

$$
\frac{\partial u}{\partial \xi}+B i(u-U)=0 \quad \text { on } \partial \Omega
$$

Here, $\lambda$ is a new dimensionless eigenvalue parameter given by 


$$
\lambda=\frac{L^{2} \rho Q A R}{k E_{a}} .
$$

In contrast with the eigenvalue $\delta, \lambda$ is decoupled from the ambient temperature. Thus, $U$ is the only parameter dependent on $T_{a}$.

The new formulation may also be put in a generalized form applicable to all three principal centrosymmetric solids. As before, we expand the Laplacian operator in cartesian, cylindrical, and spherical coordinates and observe that these can all be represented in the parameterized form:

$$
\nabla_{\xi}^{2} u=\frac{\partial^{2} u}{\partial \xi^{2}}+\frac{n}{\xi} \frac{\partial u}{\partial \xi},
$$

where $n$ is the previously defined geometry selection parameter. That is, $n=0,1$, or 2 for the slab, infinite cylinder, and sphere, respectively. Hence, the final working form of the reaction-diffusion equation for class A shapes may be written as

$$
\frac{\partial u}{\partial \tau}=\frac{\partial^{2} u}{\partial \xi^{2}}+\frac{n}{\xi} \frac{\partial u}{\partial \xi}+\lambda e^{-1 / u} \quad \text { in } \Omega
$$

and

$$
\frac{\partial u}{\partial \tau}+B i(u-U)=0 \quad \text { on } \quad \partial \Omega .
$$

This arrangement of the reaction-diffusion equation contains no simplifying approximations beyond those previously contained in equations (2) and (3) and is a mathematically equivalent framework for the analysis of stationary and nonstationary models of thermal ignition. Indeed, for numerical analyses, there is no distinct advantage in using the Frank-Kamenetskii variables and it is indeed preferable to utilize the Burnell-GrahamEagle-Gray-Wake formulation, which provides a more direct link to the physical domain. Thus, all formal developments in this work are based on the latter formulation.

In this new variable formulation, the most appropriate bifurcation control parameter is the dimensionless ambient temperature $U$ with the dimensionless reactant temperature $u$ serving as the response function. That is, $\lambda$ is normally fixed and the objective is to determine solution branches in the $(u, U)$ plane. In this case, the first bifurcation point on the minimal branch of solutions occurs at the critical ambient temperature, $U_{c r}$. Thus, the critical ambient temperature can be inferred directly from the $(u, U)$ bifurcation diagram as

$$
U_{c r i t}=\frac{R T_{a, c r i t}}{E_{a}} .
$$

Table 1 summarizes the mathematical relationship between the $(\varepsilon, \theta, \delta)$ set of variables and the $(u, U, \delta)$ set of variables as an aid to interpretation and translation between the two formulations. 
Table 1. Mathematical relationship between alternative dimensionless variables.

\begin{tabular}{|c|c|}
\hline$(\boldsymbol{\varepsilon}, \boldsymbol{\theta}, \boldsymbol{\delta}) \rightarrow(\boldsymbol{u}, \boldsymbol{U}, \boldsymbol{\lambda})$ & $(\boldsymbol{u}, \boldsymbol{U}, \boldsymbol{\lambda}) \rightarrow(\boldsymbol{\varepsilon}, \boldsymbol{\theta}, \boldsymbol{\delta})$ \\
\hline$u=\varepsilon(1+\varepsilon \theta)$ & $\theta=(u-U) / U^{2}$ \\
$U=\varepsilon$ & $\varepsilon=U$ \\
$\lambda=\delta \varepsilon^{2} e^{1 / \varepsilon}$ & $\delta=\lambda e^{-1 / U / U^{2}}$ \\
\hline
\end{tabular}

\subsubsection{Shape Factor Method for Arbitrary Convex Regions}

The basic conceptual idea of using a geometric selection parameter $n$ may be generalized to incorporate arbitrary non class A three dimensional shapes by means of the shape factor method formalized by Boddington et al., ${ }^{8}$ which may be regarded as an extension of the equivalent sphere concept introduced by Wake and Walker. ${ }^{9}$ For arbitrary convex body shapes of volume $V$ and surface area $S$, the method produces non-integral values of $n$ in terms of the Semenov radius $R_{S}$ and the harmonic mean radius $R_{0}$ :

$$
n+1=3 \frac{R_{0}^{2}}{R_{s}^{2}},
$$

where

$$
R_{S}=3 \frac{V}{S}
$$

and

$$
R_{0}^{-2}=\frac{1}{4 \pi} \iint \frac{d \omega}{a^{2}} .
$$

Here, $d \omega$ is the solid angle subtended at the center $O$, and $a$ is the radius from $O$ to a point on the surface. The dimensionless spatial variable is defined in the conventional way,

$$
\xi=\frac{a}{a_{0}}
$$

and, for class A geometries, $L$ is related to $n$ and $R_{0}$ by the formula:

$$
L=a_{0}=R_{0} \sqrt{\frac{n+1}{3}} .
$$


Table 2. Values of $R_{S}, R_{0}$, and $n$ for some common geometries.

\begin{tabular}{|l|c|c|c|}
\hline \multicolumn{1}{|c|}{ Shape } & $\mathbf{R}_{\mathbf{s}}$ & $\mathbf{R}_{\mathbf{0}}$ & $\mathbf{n}$ \\
\hline Infinite slab & 3.000 & 3.000 & 0.000 \\
Rectangular parallelepiped (1:10:10) & 2.500 & 1.731 & 0.438 \\
Infinite cylinder & 1.500 & 1.225 & 1.000 \\
Infinite square rod & 1.500 & 1.354 & 1.443 \\
Rectangular parallelepiped (1:1:10) & 1.429 & 1.354 & 1.694 \\
Sphere & 1.000 & 1.000 & 2.000 \\
Equicylinder & 1.000 & 1.115 & 2.728 \\
Cube & 1.000 & 1.194 & 3.280 \\
Regular tetrahedral & 0.408 & 0.537 & 4.187 \\
\hline
\end{tabular}

A summary of results for class A and non class A shapes, as computed by Boddington et al, ${ }^{8}$ is provided in table 2. This method has been extended to the revised variable formulation and applied to an extensive study of the stationary model by Balakrishnan. ${ }^{10}$ This was accomplished by applying pathfollowing techniques to the stationary form of equation (19) with the eigenvalue parameter $\bar{\lambda}$. The value of $\lambda$ for non class A geometries of unit size is recovered by scaling $\bar{\lambda}$, using the relation

$$
\lambda=\frac{3}{(n+1) R_{0}^{2}} \bar{\lambda} .
$$

It is believed that this method is equally applicable to the nonstationary model and might prove practical for the estimation of critical initial conditions in assembly problems. 


\section{STATIONARY MODEL}

Historically, greater emphasis has been placed on theoretical understanding and mathematical modeling of the stationary model than the nonstationary model, because it reduces the determination of explosion or thermal ignition to a consideration of possible steady-state solutions of a standard nonlinear eigenvalue problem. These types of second-order ordinary differential equations have been extensively studied, both mathematically and computationally, and excellent reviews and compilations are available in the literature. ${ }^{11,12}$ Such studies ordinarily adopt the Frank-Kamenetskii variables and examine the behavior of $\theta$ using $\delta$ as a bifurcation parameter. Consequently, attention has been focused almost exclusively on determination of $\delta_{c r}$, the first bifurcation point on the minimal branch, which has direct practical implications to the storage problem but only limited utility, at best, to the assembly problem.

Balakrishnan, in one of the first attempts to utilize the Burnell-GrahamEagle-Gray-Wake variables, revisited the classic stationary model and carried out extensive numerical analyses of the eigenvalue problem using path-following techniques. ${ }^{10}$ This work illuminated the characteristic branch structure in the $(u, U)$ plane, including accurate identification of $U_{c r}$, and convincingly demonstrated that these alternative variables provide improved physical clarity and more straightforward interpretation in terms of the ambient temperature control parameter.

The major focus of this thesis, following the initial lead of Weber et al., ${ }^{4}$ will be the solution of the nonstationary model in alternative variable form and determination of critical initial conditions in the oft neglected but practically important assembly problem. In doing so, it will first be necessary to construct stationary model solutions to provide a frame of reference for proper interpretation of the computed critical initial condition bifurcation branches. Thus, a numerical analysis procedure is developed herein for the standard stationary problem in alternative variable form. Rather than follow the sophisticated path following method outlined by Balakrishnan, ${ }^{10}$ however, a more direct and less laborious route is taken whereby the conventional two-point boundary value problem is reformulated as an equivalent initial value problem. Before embarking on this development for the stationary model, it will prove useful to first review the qualitative solution behavior for the general eigenvalue problem as obtained from the time-independent form of equation (15):

$$
\nabla_{\xi}^{2} u+\lambda e^{-1 / u}=0 \quad \text { in } \Omega
$$

where the boundary condition defined by equation (16) still applies.

\subsection{Qualitative Structure of Solution Branches}

Consider the anticipated functional behavior of heat generation and heat loss in a porous pile of material undergoing chemical oxidation within a fixed temperature ambient environment. First, we note that the heat loss rate is assumed to be proportional to the temperature difference between the body and 
the ambient environment and would therefore have a linear functional dependence on body temperature, as illustrated by the dashed lines in figure 1 corresponding to $T_{a}<T_{a, c r}, T_{a}=T_{a, c r}$ and $T_{a}>T_{a, c r}$. On the other hand, we clearly expect the heat generation rate to vary in a nonlinear way with increasing body temperature. At relatively low body temperature when the driving potential for heat loss is low, the heat generation would tend to increase strongly with any incremental rise in temperature, whereas at high body temperature, when the driving potential for heat loss is higher and diffusion becomes a controlling factor, the heat generation rate would exhibit a weaker response to any incremental increase in temperature. Thus, the functional dependence of heat generation rate on body temperature would tend to follow a sigmoid type curve as illustrated by the solid curve in figure 1 . The intersection points of the heat generation and loss curves represent the steady state solutions we seek for the stationary storage problem.

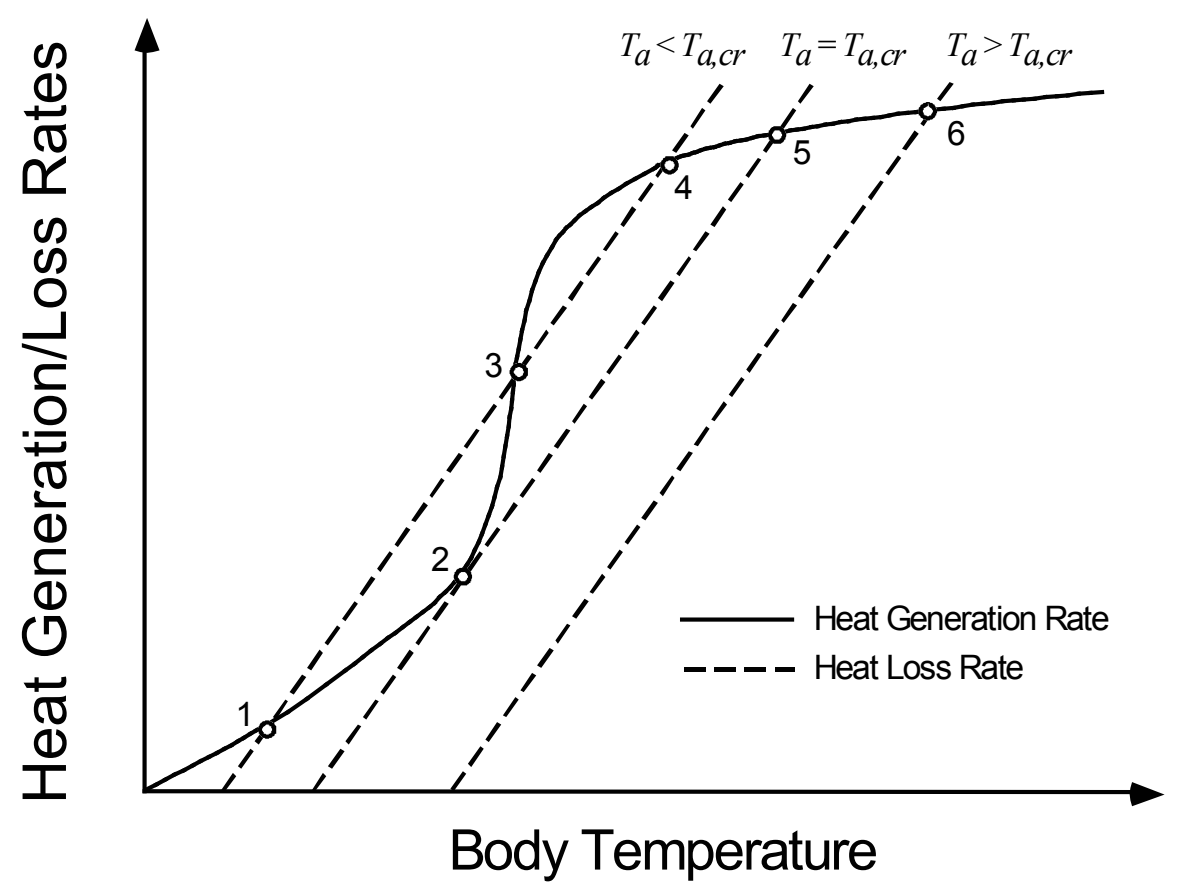

Figure 1. Heat balance characteristics for the stationary ignition model.

When $T_{a}<T_{a, c r}$, the body is able to efficiently eject heat to the environment at a relatively high rate, and we obtain three steady-state solutions at intersection points 1,3 , and 4 . For this ambient condition, the stationary model implies that the body temperature will always approach $T_{1}$ if $T_{i}<T_{3}$ and $T_{4}$ if $T_{i}>T_{3}$. Here, $T_{i}$ is the initial assembly temperature. The intermediate intersection point at $T_{3}$ is an unstable steady state and cannot be realized. When $T_{a}=T_{a, c r}$, the body ejects heat at a reduced rate, and we now obtain two steady-state solutions at intersection points 2 and 5. At this critical ambient temperature, the stationary model implies that the body temperature will always approach $T_{2}$ whenever $T_{i}<T_{2}$ and $T_{5}$ whenever $T_{i}>T_{2}$. When $T_{a}>T_{a, c r}$, however, the ability to eject heat is significantly curtailed, and we obtain a single steady-state solution at intersection point 6 . Thus, the body temperature will always approach $T_{6}$ for any initial value of $T_{i}$. In most practical cases, the upper branch of steady-state solutions corresponds to temperatures in excess of the material flame temperature, and ignition will occur before those conditions can 
be attained. Therefore, the upper branch serves as a nonphysical mathematical attractor only. In reality, the stationary model neglects all transient effects and is only applicable to storage problems where $T_{i}$ is well below the intermediate steady-state solution. It cannot be used to reliably predict a safe assembly temperature.

There are certain mathematically significant conclusions of fundamental importance to be noted from this qualitative inspection of the stationary reaction-diffusion equation:

- At least one steady-state solution exists for any finite value of physical parameters when $T_{a}>0$.

- A multiplicity of steady-state solutions may exist whenever $0<T_{a}<T_{a, c r}$. When $T_{a}<T_{a, c r}$, the body of reacting material will either tend to a stable lower steady-state temperature below the ignition point or to an upper steady state temperature above the ignition temperature. The critical material assembly temperature, $T_{i, c r}$, demarking the two possible responses can only be roughly estimated using the unstable intermediate steady state solution branch. Accurate determination of $T_{i, c r}$ requires consideration of the fully transient reaction-diffusion equation.

- When $T_{a}=T_{a, c r}$, two steady state solutions exist. The lower solution corresponds to the first bifurcation point at the intersection point of the lower and intermediate steady-state branches, and the upper steady-state temperature exceeds the value required for ignition.

- When $T_{a}>T_{a, c r}$, only one solution exists and the body will always tend to the upper steady-state branch, in which case ignition is assured.

The characteristic behavior of the steady-state solution is also dependent on variations in the value of $\lambda .{ }^{13}$ If $\lambda$ is small, a unique solution always exists. If $\lambda$ is sufficiently large, multiple solutions exist whenever $T_{a}<T_{a, c r}$. When $T_{a}>T_{a, c r}$, a unique solution exists for any value of $\lambda$. The transitional value of $\lambda$ above which multiplicity first occurs is denoted as $\lambda_{t r}$. When $U=0$, the transitional value of $\lambda$ above which multiplicity first occurs is denoted as $\lambda^{\prime}$.

\subsection{Numerical Methodology}

The possibilities for constructing analytical solutions to the stationary reaction-diffusion equation in the dimensionless set of variables are extremely limited for even the simplest shaped regions. Thus, numerical methods are required to obtain accurate solutions and reliable prediction of the critical parameter values. The mathematical object of consideration is the solution set $\{(u, U, \lambda)\}$ to the second-order nonlinear ordinary differential equation for arbitrary shape factor $n$

$$
\frac{d^{2} u}{d \xi^{2}}+\frac{n}{\xi} \frac{d u}{d \xi}+\lambda e^{-1 / u}=0, \quad 0 \leq \xi \leq 1
$$

subject to the following boundary conditions at the axis of symmetry and the body surface: 


$$
\left.\frac{d u}{d \xi}\right|_{\xi=0}=0 \quad \text { and }\left.\quad \frac{d u}{d \xi}\right|_{\xi=1}+B i\left(u_{s}-U\right)=0
$$

where $u_{s}$ is the dimensionless temperature at the body surface.

\subsubsection{Reduction to First-Order ODE System}

The numerical solution of equation (29) is facilitated by reduction to a first-order system of differential equations. By defining $u_{1}=u$ and $u_{2}=d u_{1} / d \xi$, for example, we obtain the first-order system

$$
\begin{aligned}
& \frac{d u_{1}}{d \xi}=u_{2} \\
& \frac{d u_{2}}{d \xi}=-\frac{n}{\xi} u_{2}-\lambda e^{-1 / u_{1}}
\end{aligned}
$$

Note that this system is nonautonomous since $\xi$ appears explicitly in the denominator, and there is an apparent singularity at the origin, even though a solution must exist at that point. The singularity can be removed, however, by observing that when $\xi$ approaches zero,

$$
u_{2}^{\prime}(\xi)=u_{1}^{\prime \prime}(\xi)=\lim _{\xi \rightarrow 0} \frac{u_{1}^{\prime}(\xi)-u_{1}^{\prime}(0)}{\xi} \approx \frac{u_{1}^{\prime}(\xi)}{\xi}=\frac{u_{2}}{\xi},
$$

since $u_{1}{ }^{\prime}(0)=0$. This approximation may then be used to eliminate $u_{2}$ in equation (31) as $\xi \rightarrow 0$ :

$$
\frac{d u_{2}}{d \xi}=-n \frac{d u_{2}}{d \xi}-\lambda e^{-1 / u_{1}} \quad \text { as } \xi \rightarrow 0
$$

which gives

$$
\frac{d u_{2}}{d \xi}=\frac{\lambda}{n+1} e^{-1 / u_{1}} \quad \text { as } \xi \rightarrow 0
$$

Thus, we may redefine the first-order system, equation (31), in piecewise form as

$$
\begin{aligned}
& \frac{d u_{1}}{d \xi}=u_{2} \\
& \frac{d u_{2}}{d \xi}= \begin{cases}-\frac{\lambda}{n+1} e^{-1 / u_{1}}, & \xi \leq \varsigma \\
-\frac{n}{\xi} u_{2}-\lambda e^{-1 / u_{1}}, & \xi>\varsigma\end{cases}
\end{aligned}
$$

where $\zeta$ is a finite value very close to zero. Transformation of the boundary conditions defined by equation (30) yields the relations: 


$$
u_{1}(1)=U-u_{2}(1) / B i \quad \text { and } \quad u_{2}(0)=0
$$

\subsubsection{Disjointedness and Redefinition}

The first-order system, equation (35), with boundary conditions, equation (36), defines a nonlinear two-point boundary value problem which may be solved using various sophisticated path-following methods. A serious numerical difficulty arises, however, when the value of $\lambda$ increases beyond $\lambda^{\prime}$ and the extinction point - i.e., the upper limit point in the bifurcation diagram - is moved into the physically unacceptable domain of negative $U$ values. ${ }^{13,14}$ In this case, the upper and lower branches of solutions become disjoint in the physically acceptable region where $U>0$. Furthermore, Balakrishnan has demonstrated that there can be no solutions with bounded derivatives in the case $U<0$ for which $u(r)$ changes sign in the region $0 \leq \xi \leq 1.10$

A straightforward approach for making a connection between the upper and lower branches of solution curves in the $U<0$ region is to simply switch off the source term. ${ }^{10}$ Mathematically, this may be formally accomplished through inclusion of the Heaviside function, $H(u)$, as a multiplicative factor to the source term, in which case,

$$
\frac{d^{2} u}{d \xi^{2}}+\frac{n}{\xi} \frac{d u}{d \xi}+\lambda H(u) e^{-1 / u}=0, \quad 0 \leq \xi \leq 1
$$

where

$$
\begin{array}{ll}
H(u)=1, & u>0, \\
H(u)=0, & u \leq 0 .
\end{array}
$$

Thus, the disjointedness may be removed and the system made autonomous by introducing the new variable $u_{3}=\xi$ and redefining the piecewise first-order system, equation (31), as follows:

$$
\begin{aligned}
& \frac{d u_{1}}{d \xi}=u_{2} \\
& \frac{d u_{2}}{d \xi}= \begin{cases}-\frac{\lambda}{n+1} e^{-1 / u_{1}}, & u_{3} \leq \varsigma \text { and } u_{1}>0 \\
0, & u_{3} \leq \varsigma \text { and } u_{1} \leq 0 \\
-n \frac{u_{2}}{u_{3}}-\lambda e^{-1 / u_{1}}, & u_{3}>\varsigma \text { and } u_{1}>0 \\
-n \frac{u_{2}}{u_{3}}, & u_{3}>\varsigma \text { and } u_{1} \leq 0\end{cases} \\
& \frac{d u_{3}}{d \xi}=1
\end{aligned}
$$

with the boundary conditions

$$
u_{1}(1)=U-u_{2}(1) / B i, \quad u_{2}(0)=0, \quad \text { and } \quad u_{3}(0)=0
$$




\subsubsection{Path-Following Method}

A numerical procedure is now needed to path follow the solution curves for the stationary thermal ignition model. Standard path-following techniques (viz., piecewise linear or predictor-corrector methods) are available to handle this problem, ${ }^{15-22}$ but we shall develop a more straightforward approach based on direct numerical integration of the first-order system, equation (39), with boundary conditions, equation (40). Although this procedure will lack the sophistication of standard methodologies, it will be seen to be an efficient and highly effective means for constructing accurate bifurcation branch diagrams for the steady-state ignition problem.

The central technical difficulty is that we are faced with a two-point boundary value problem, since $u_{1}(0)=u(0)$, the dimensionless center-point temperature, is not known a priori. Otherwise, the system of equations could be readily solved as an initial value problem using conventional numerical integration techniques. Therefore, a simple ad hoc procedure has been adapted in which the solution branches in the $\{u(0), U\}$ plane, with $U$ as the bifurcation parameter, can be constructed from the solution of an equivalent initial value problem.

The procedure is as follows:

- Take $u_{O}$ as an independent parameter for the dimensionless center-point body temperature such that

$$
0 \leq u_{O} \leq u_{O, \max }
$$

- Specify a value for $\lambda$ and use a standard fourth-order Runge-Kutta algorithm to numerically solve system equation (39) as an initial value problem from $\xi=0 \rightarrow 1$ with the initial conditions defined by

$$
u_{1}(0)=u_{O}, \quad u_{2}(0)=0, \quad \text { and } \quad u_{3}(0)=0
$$

- Use the computed boundary values $u_{1}(1)$ and $u_{2}(1)$ from the preceding step along with the specified Biot number to compute the corresponding body surface temperature from the boundary condition

$$
U=u_{1}(1)+u_{2}(1) / B i
$$

- Repeat the procedure above for incremental values of $u_{O}$ over the range $0 \rightarrow u_{0 \text {,max }}$ and path follow the solution branch $u(0)=u_{O}$ versus the bifurcation parameter $U$.

\subsection{Validation}

An extensive numerical investigation of the stationary model was previously carried out and thoroughly documented by Balakrishnan using conventional path-following tools, ${ }^{10}$ and his results are now adopted as a benchmark validation standard for the ad hoc path-following procedure described above. Thus, the solution sets $\{(u, U, \lambda)\}$ are examined and validated for the principal centrosymmetric solids $(n=0,1,2)$, keeping $U$ as the bifurcation parameter and allowing $\lambda$ and $B i$ to vary.

For illustrative purposes, representative bifurcation diagrams were computed using the above pathfollowing procedure for various values of $\lambda$, assuming an infinite Biot number. These results are shown in 
figures 2, 3, and 4 for $n=0,1,2$, respectively. It is found that these predictions are in excellent agreement with Balakrishnan's calculations, which were obtained using more sophisticated methods and standard algorithms. ${ }^{23}$ Both methods yield identical values of $\lambda_{t r}$ and $\lambda^{\prime}$ for all three geometric shapes, as indicated in the accompanying figures, and the corresponding critical dimensionless ambient temperatures $U_{c r}$ are in complete accord.

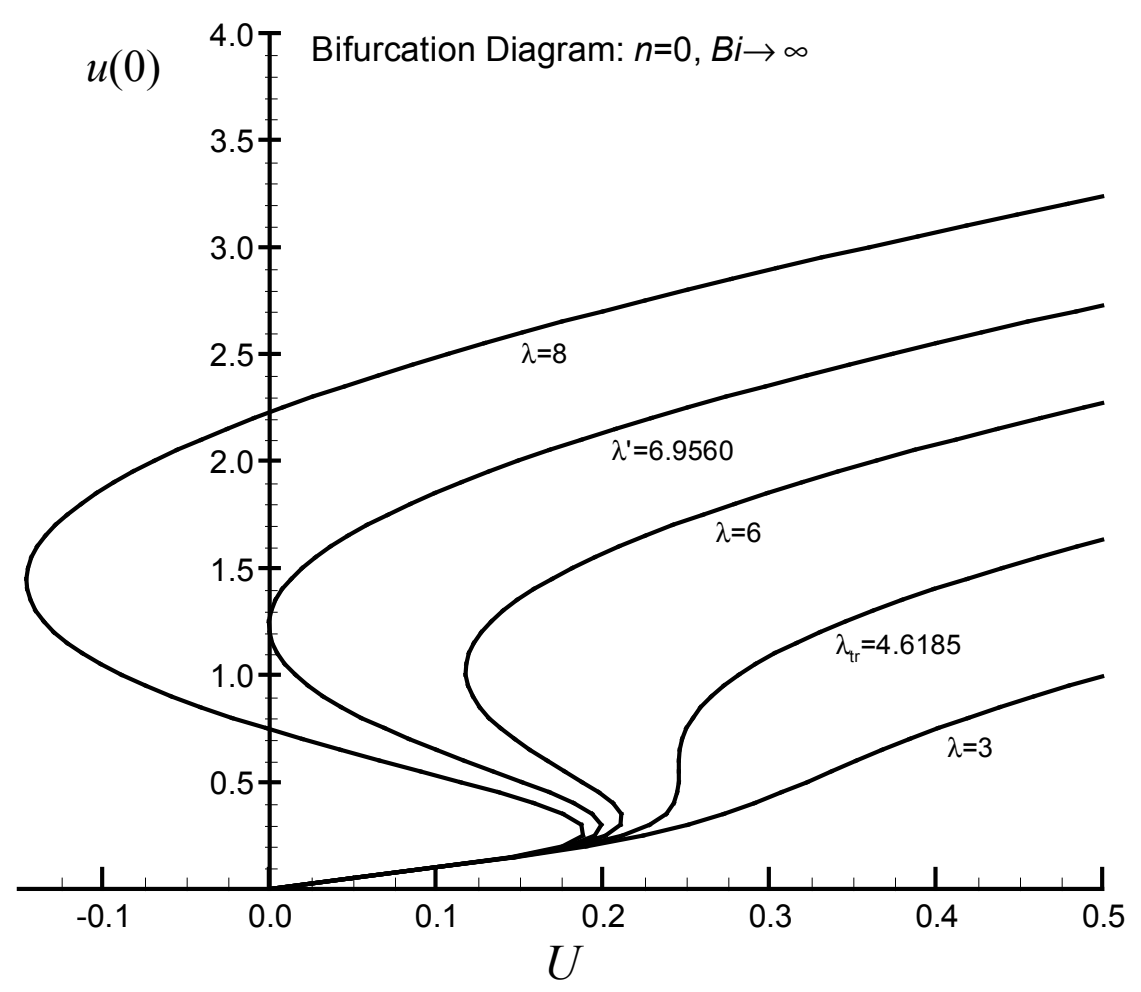

Figure 2. Computed bifurcation diagram for the infinite slab $(n=0)$ with $B i \rightarrow \infty$.

As a further validation step, comparative calculations were completed for $U_{c r}$ and $u(0)$ for the principal centrosymmetric solids $(n=0,1,2)$, keeping $U$ as the bifurcation parameter and allowing $\lambda$ and $B i$ to vary. The variation in the critical parameters against $\lambda$ are shown in figures 5 and 6 under the constraint $B i \rightarrow \infty$. The variation in the critical parameters against $B i$ are shown in figures 5 and 6 under the constraint $\lambda=50$. These results are all in excellent agreement with previously established theoretical predictions. For infinite $B i$, it is observed that $U_{c r}$ and $u(0)$ both decrease at the ignition point with increasing $\lambda$. For a fixed value of $\lambda$, we observe that $U_{c r}$ and $u(0)$ both increase with increasing $B i$ and asymptotically approach limiting values as $B i \rightarrow \infty$ (figures 7 and 8 ).

Because the predictions were found to be precise and accurate, under all circumstances, in comparison to established theoretical calculations, it is asserted that the results from the proposed ad hoc path-following method are fully valid and may be used as a reliable benchmark standard for the stationary model. Thus, results of the theoretical investigations to follow may be referred to this baseline with a high degree of confidence. 


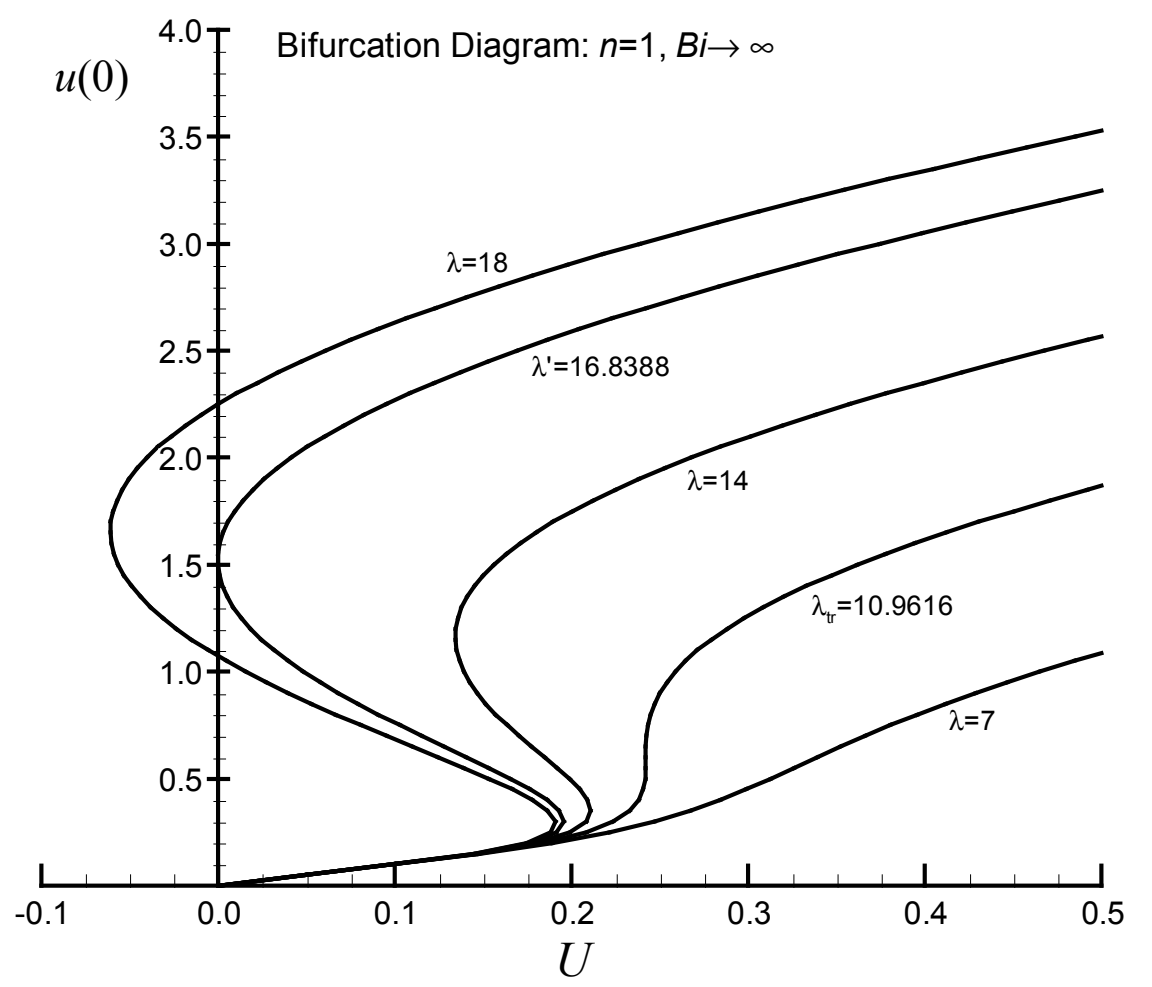

Figure 3. Computed bifurcation diagram for the infinite cylinder $(n=1)$ with $B i \rightarrow \infty$.

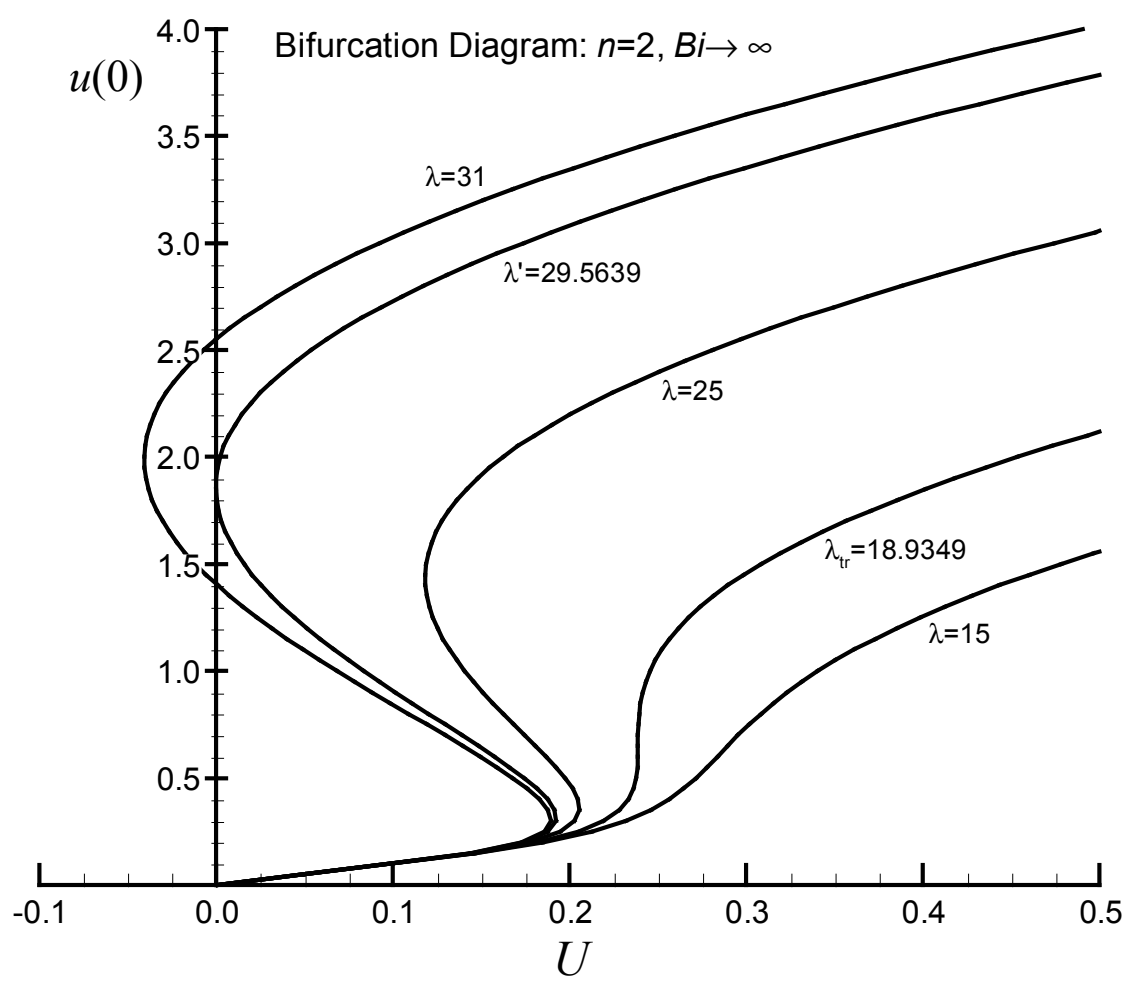

Figure 4. Computed bifurcation diagram for the sphere $(n=2)$ with $B i \rightarrow \infty$. 


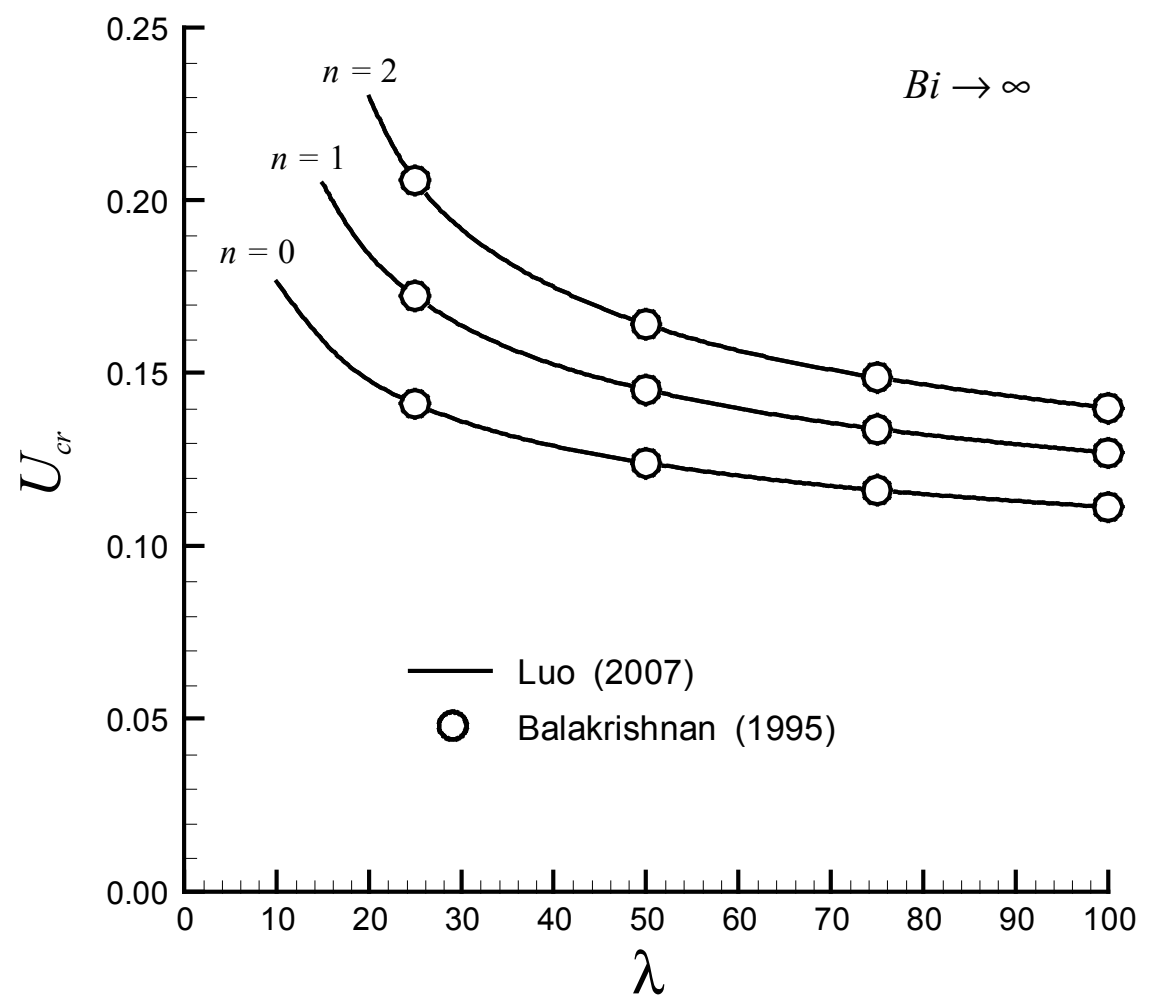

Figure 5. Variation of $U_{c r}$ against $\lambda$ for $n=0,1,2$ with $B i \rightarrow \infty$.

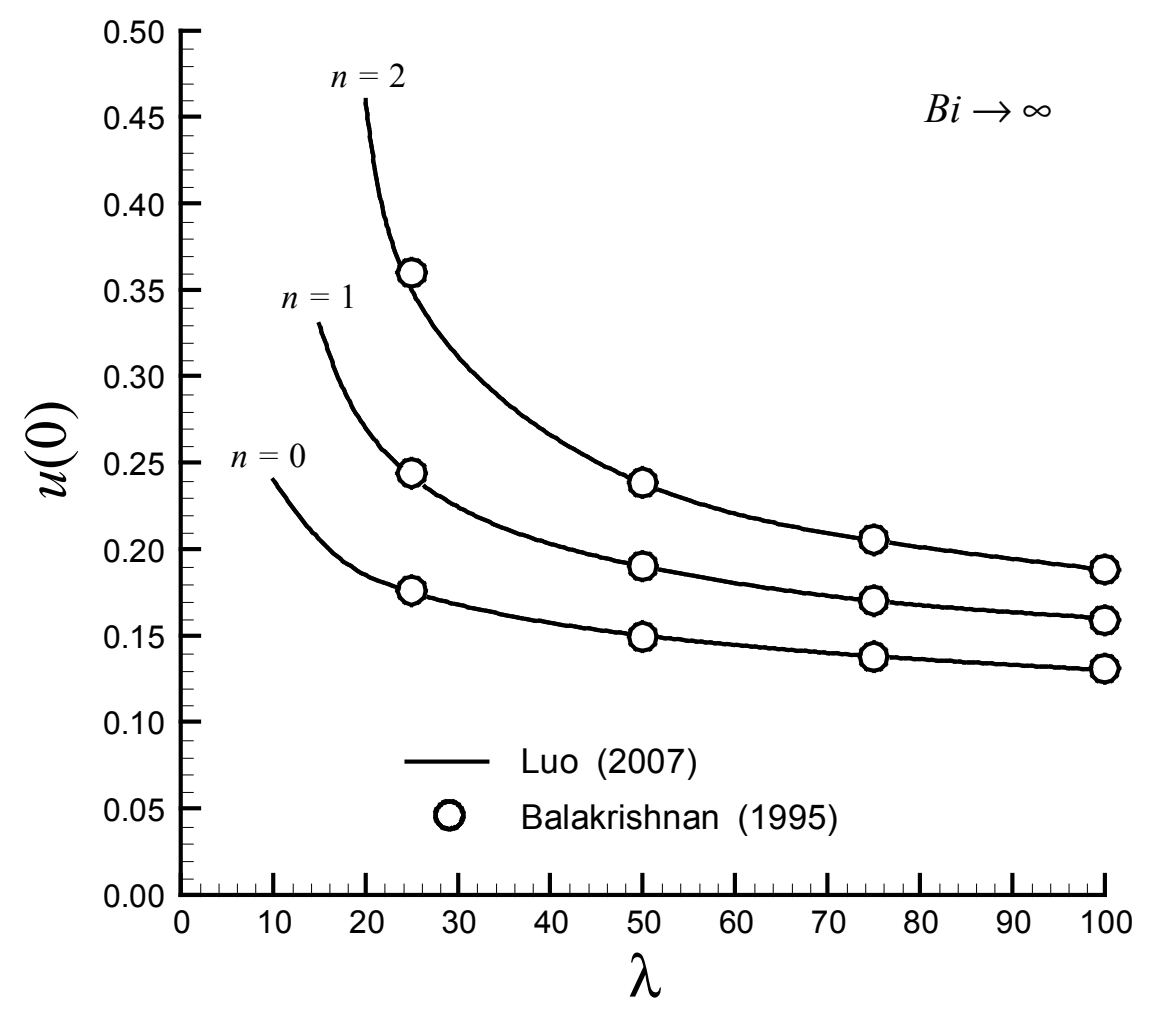

Figure 6. Variation of $u(0)$ against $\lambda$ for $n=0,1,2$ with $B i \rightarrow \infty$. 


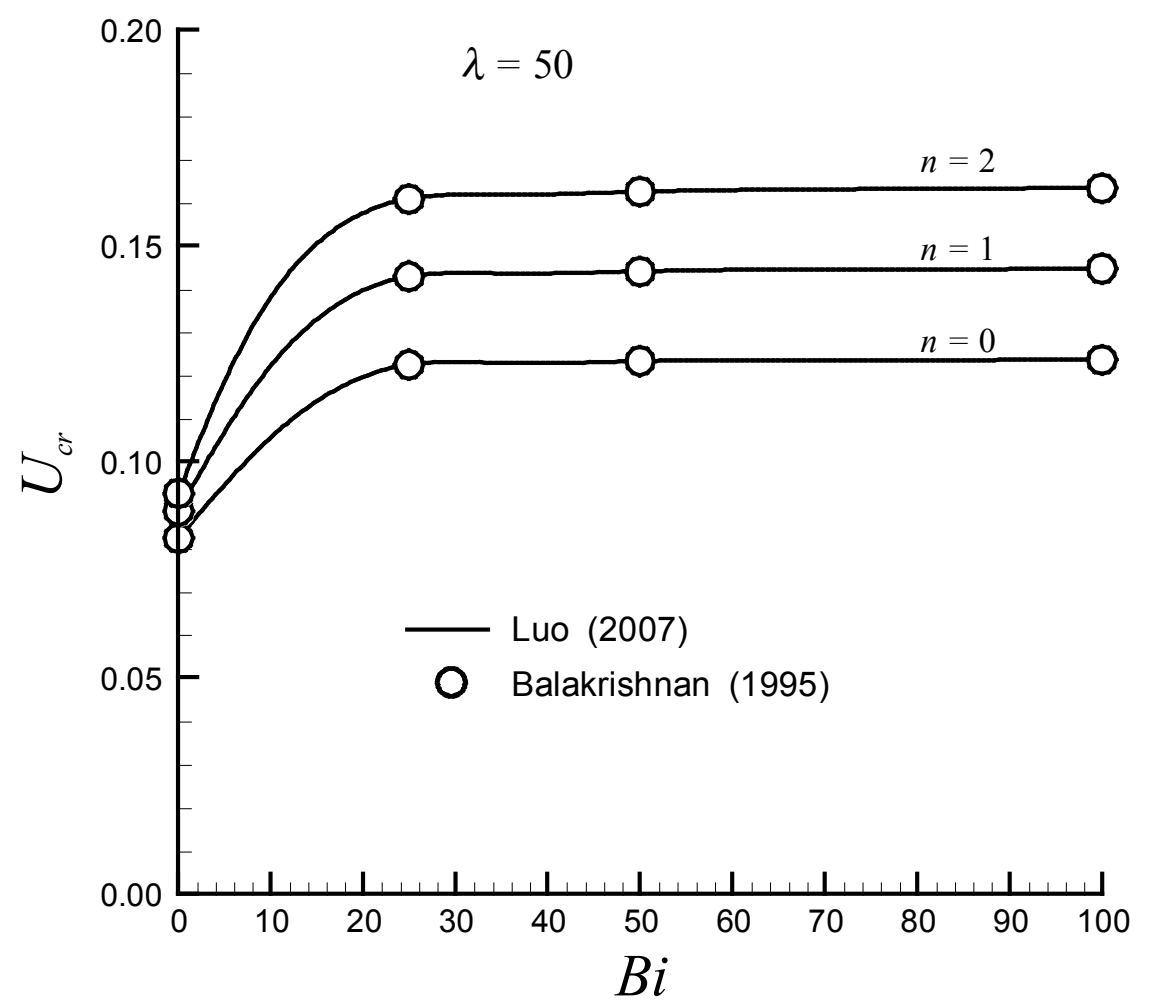

Figure 7. Variation of $U_{c r}$ against $B i$ for $n=0,1,2$ with $\lambda=50$.

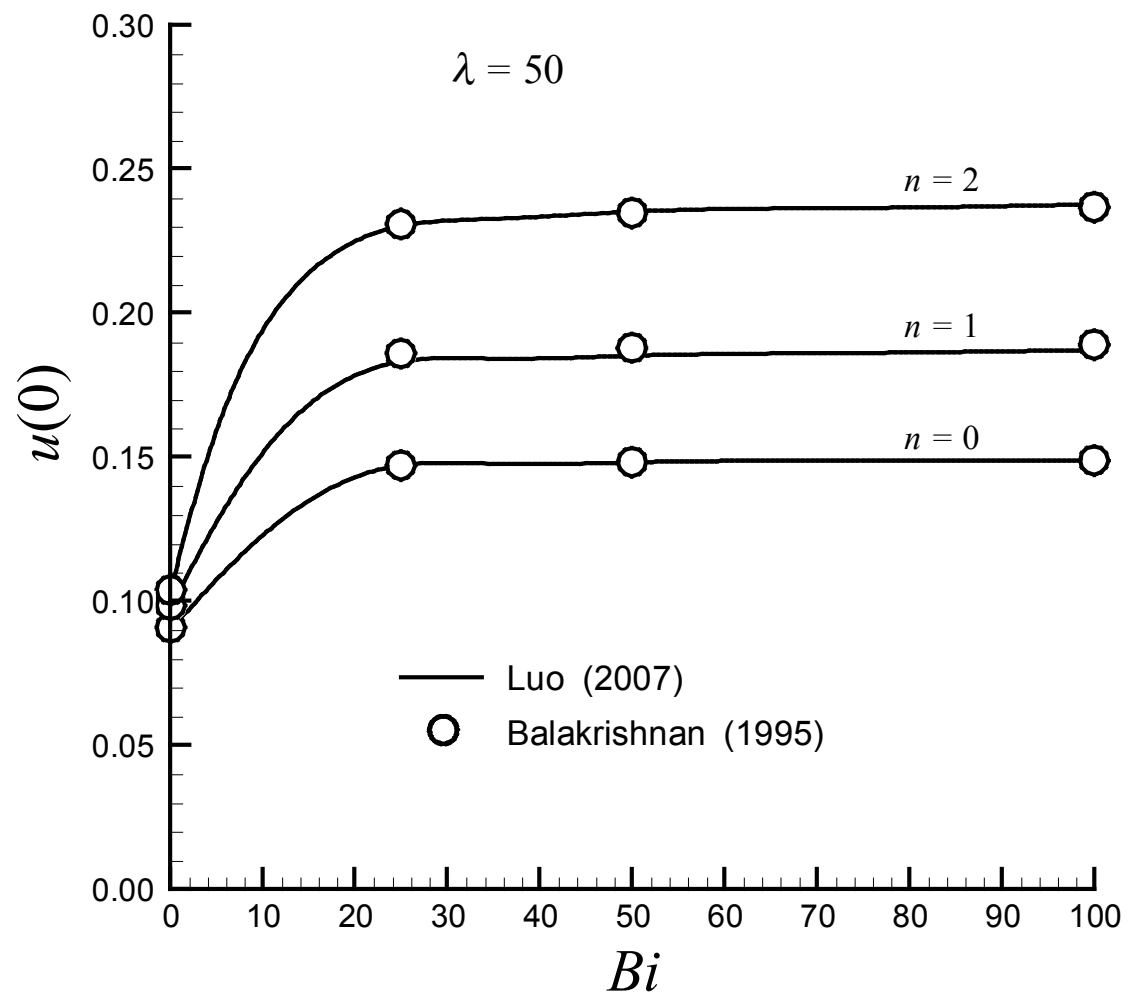

Figure 8. Variation of $u(0)$ against $B i$ for $n=0,1,2$ with $\lambda=50$. 
In most practical cases, the value of $\lambda$ is orders of magnitude higher than the typical range associated with $\lambda_{t r}$ and $\lambda^{\prime}$. Flammable materials of common interest, for instance, will normally fall in some range around $\lambda \approx 10^{6}$. Therefore, the bifurcation diagrams for the principal centrosymmetric solids have been constructed for $\lambda=10^{4}, \lambda=10^{6}$, and $\lambda=10^{8}$ with $B i \rightarrow \infty$, and these results, summarized in figures 9-11, will be used as a general frame of reference in the theoretical investigations of the nonstationary model to follow.

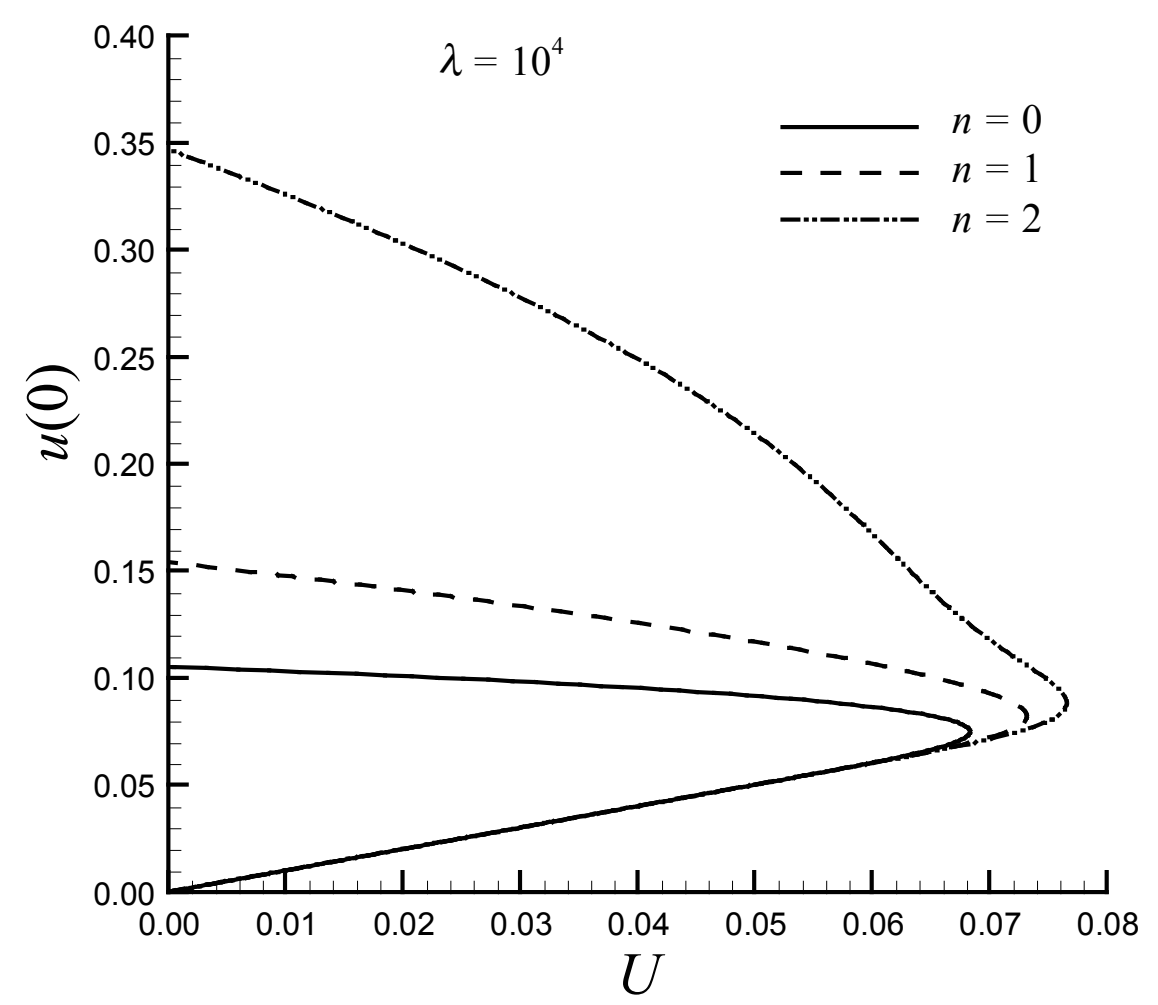

Figure 9. Computed bifurcation diagrams with $\lambda=10^{4}$ and $B i \rightarrow \infty$. 


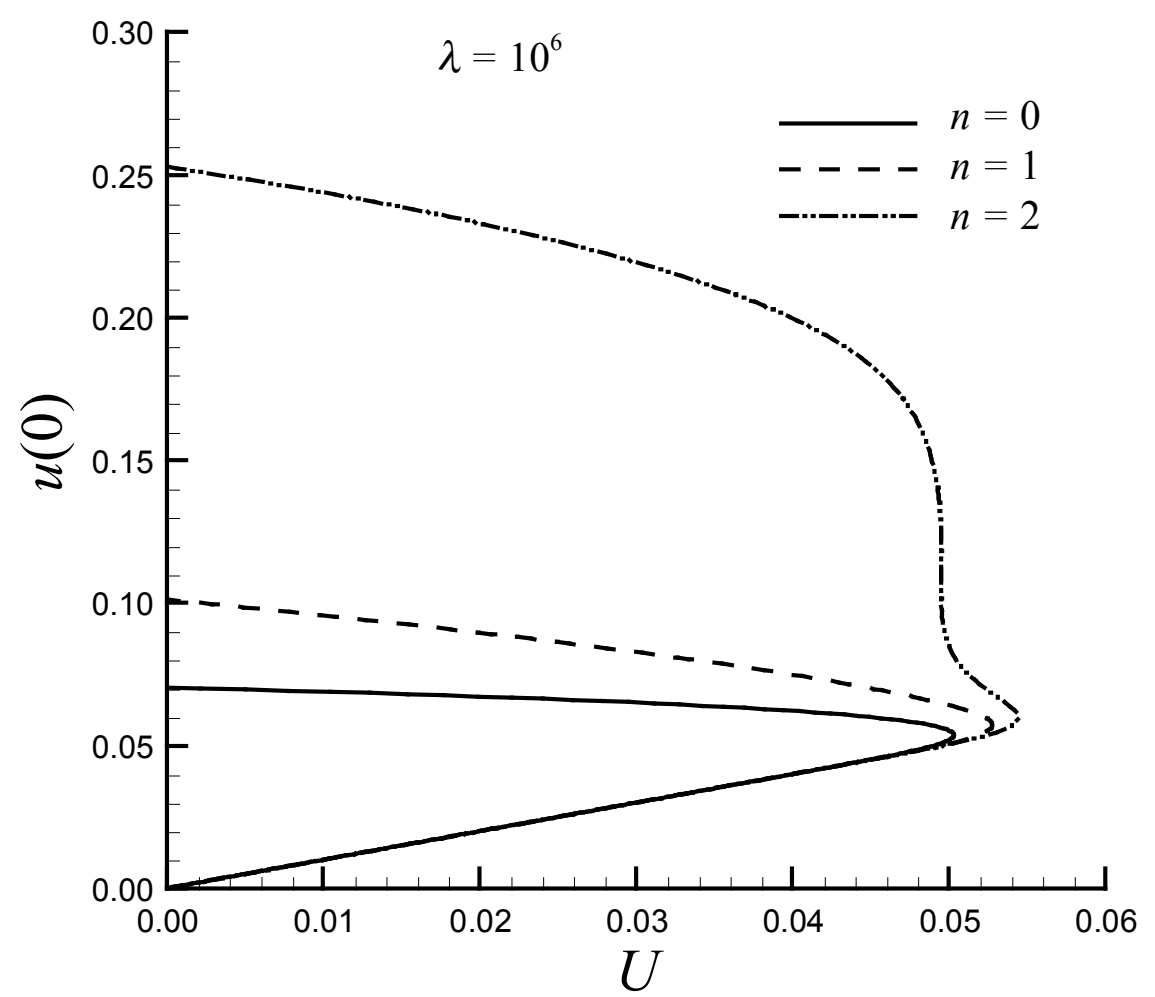

Figure 10. Computed bifurcation diagrams with $\lambda=10^{6}$ and $B i \rightarrow \infty$.

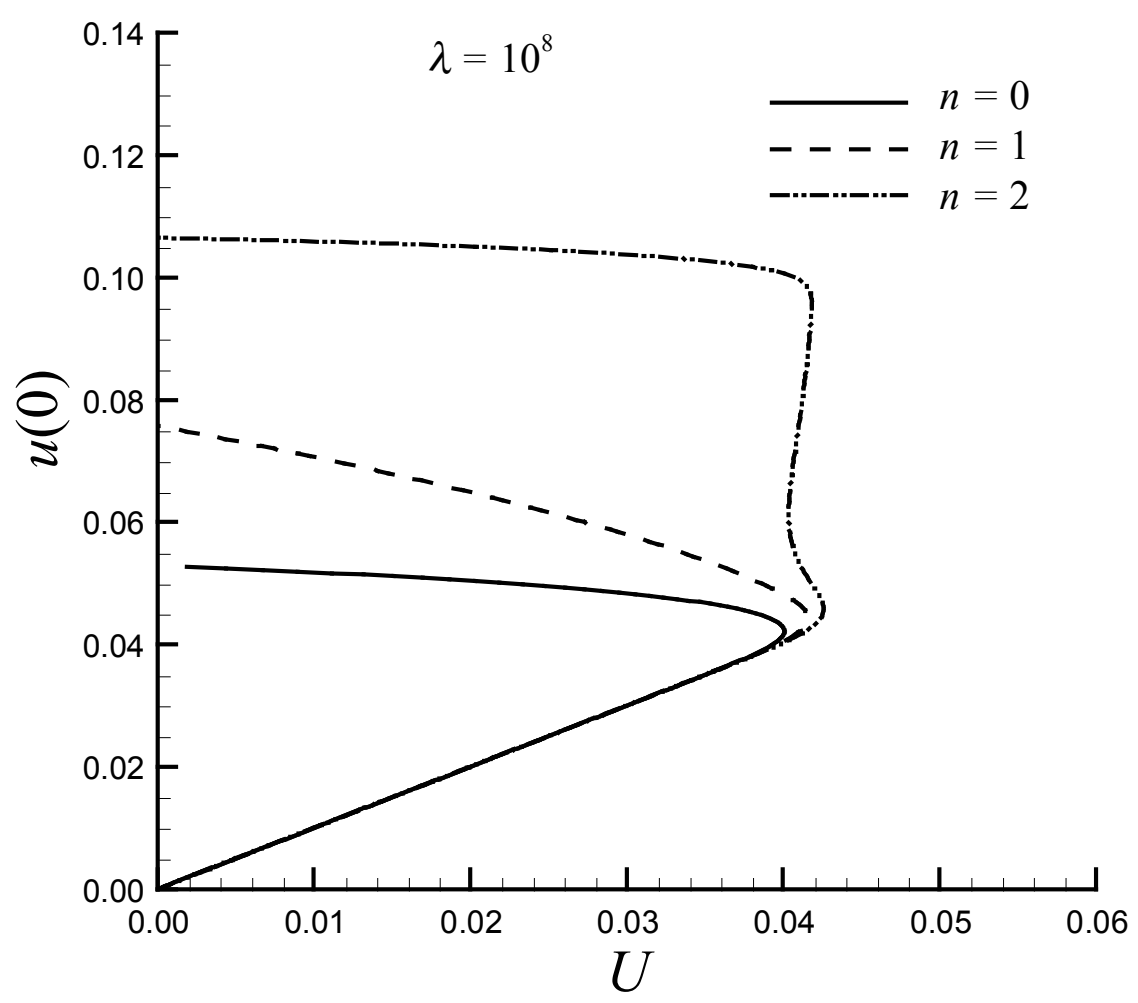

Figure 11. Computed bifurcation diagrams with $\lambda=10^{8}$ and $B i \rightarrow \infty$. 


\section{NONSTATIONARY MODEL}

Real world problems, such as the safe assemblage of hot reactive materials and so-called 'hot spot' ignition, where localized high temperature regions expand and initiate thermal explosion, can be significantly dependent on the contribution of transient heating processes. Thus, the fate of a self-heating material depends upon circumstances of assembly and external environment, and the most indiscriminate case requires consideration of the fully transient reaction-diffusion equation.

Exact analytical constructions for the generalized nonstationary model have proved evasive, however, and progress along these lines has been mainly limited to less general problem formulations derived from simplifications to the nonlinear Arrhenius rate term. The elementary treatment of Frank-Kamenetskii, for example, reveals salient features of the solution, ${ }^{5}$ and Gray and Harper have developed more exact analytical representations using a quadratic approximation for the Arrhenius temperature dependence. ${ }^{24,25}$ More rigorous analytical work using expansion procedures in the region $\varepsilon<<1$ has provided accurate self-heating and explosion solutions over a time span ranging from initiation to completion. ${ }^{26-29}$ For a complete comprehensive treatment valid over the entire parameter range, however, numerical integration techniques are required.

As recourse, we embrace a numerical approach to the Burnell-GrahamEagle-Gray-Wake form of the transient reaction-diffusion problem for class A shapes, as derived in section 1 and repeated here for convenience:

$$
\begin{gathered}
\frac{\partial u}{\partial \tau}=\frac{\partial^{2} u}{\partial \xi^{2}}+\frac{n}{\xi} \frac{\partial u}{\partial \xi}+S \quad \text { in } \quad \Omega \\
\frac{\partial u}{\partial \xi}+B i(u-U)=0 \quad \text { on } \partial \Omega,
\end{gathered}
$$

where we have introduced the parameter $S=\lambda e^{-1 / u}$ to represent the source term.

In proceeding, it is recognized that the reliability of computational solutions to partial differential equations is highly dependent on the integrity of the numerical scheme and the attention to detail and degree of care associated with its implementation. Therefore, the numerical methodology and implementation procedures to be used in this study are thoroughly explained, developed, and validated as a major point of departure.

\subsection{Numerical Methodology}

A numerical solution of the transient reaction-diffusion equation, which is parabolic in time and elliptic in space, consists of a finite set of numbers from which the spatial distribution of the dependent 
variable can be constructed at some instant in time. Thus, a numerical discretization methodology rests on the construction of a set of linear algebraic equations for unknown dependent variable values at a finite number of spatial locations - i.e., nodes or grid points - and the prescription of an algorithm for solving the algebraic system and advancing the solution over discrete increments of time.

Systematic spatial discretization is accomplished by introducing finite difference expressions whereby the value of the dependent variable at each node is related to the values at a small number of neighboring grid points, only. The number of neighboring values included in the finite difference expression, commonly referred to as the nodal support, determines the numerical accuracy of the discrete spatial derivative. A common systematic approach for temporal discretization, which relies on the one-way transmission of information in time, is to simply relate the initial dependent variable distribution to an evolved distribution over some small positive time increment.

\subsubsection{Generalized Discretization Equation}

3.1.1.1 Interior Nodes. Since very high order accuracy is not essential for this problem, the spatial derivatives in the dimensionless reaction-diffusion equation are approximated as three-node support finite difference expressions via truncated Taylor series. For equally spaced grid points, the Taylor series expansion about any node $i$ may be truncated after the third term to yield the following well known central difference approximations exhibiting second-order spatial accuracy:

$$
\begin{gathered}
\left.\frac{\partial u}{\partial \xi}\right|_{i} \approx \frac{u_{i+1}-u_{i-1}}{2 \Delta \xi}+o\left(\Delta \xi^{2}\right) \\
\left.\frac{\partial^{2} u}{\partial \xi^{2}}\right|_{i} \approx \frac{u_{i+1}+u_{i-1}-2 u_{i}}{\Delta \xi^{2}}+o\left(\Delta \xi^{2}\right) .
\end{gathered}
$$

Introduction of these relations into the continuum equation and temporal discretization over a small time increment $\Delta \tau$ produces a generalized discretization equation centered on each interior node $i$ :

$$
\begin{aligned}
\frac{u_{i}^{m+1}-u_{i}^{m}}{\Delta \tau} & =f\left[\frac{u_{i+1}^{m+1}+u_{i-1}^{m+1}-2 u_{i}^{m+1}}{\Delta \xi^{2}}\right]+f\left(\frac{n}{\xi_{i}}\right)\left[\frac{u_{i+1}^{m+1}-u_{i-1}^{m+1}}{2 \Delta \xi}\right]+f S_{i}^{m+1} \\
& +(1-f)\left[\frac{u_{i+1}^{m}+u_{i-1}^{m}-2 u_{i}^{m}}{\Delta \xi^{2}}\right]+(1-f)\left[\frac{n}{\xi_{i}}\right)\left[\frac{u_{i+1}^{m}-u_{i-1}^{m}}{2 \Delta \xi}\right]+(1-f) S_{i}^{m} .
\end{aligned}
$$

Note that subscripts and superscripts denote spatial and temporal indices, respectively, and that $f$ is a weighting factor $(0 \leq f \leq 1)$, which determines the relative influence of initial and final time step values during the temporal evolution process.

For certain specific values of the weighting factor $f$, the discretization equation reduces to one of the well known integration schemes for parabolic partial differential equations, as summarized in table 3 . 
Table 3. Relationship between weighting factor and integration scheme.

\begin{tabular}{|c|l|}
\hline Weighting Factor, $\boldsymbol{f}$ & \multicolumn{1}{|c|}{ Scheme } \\
\hline 0.0 & Fully explicit \\
0.5 & Crank-Nicholson \\
1.0 & Fully implicit \\
\hline
\end{tabular}

The fully explicit scheme expresses the value of the unknown dependent variable at node $i$ and future time step $m+1$ explicitly in terms of known neighboring values at the initial time step $m$. Conversely, the fully implicit scheme expresses the value of the unknown dependent variable at node $i$ and future time step $m+1$ implicitly in terms of the unknown neighboring values at the future time step $m+1$. As a middle path, the Crank-Nicholson scheme expresses the value of the unknown dependent variable at node $i$ and future time step $m+1$ partly in terms of known neighboring values at the initial time step $m$ and partly in terms of the unknown neighboring values at the future time step $m+1$.

It should be pointed out that the fully explicit scheme is prone to numerical instabilities and requires the utilization of extremely small time increments to obtain physically realistic results. It is, for all practical purposes, not useful for serious calculations. The fully implicit scheme, on the other hand, has the distinct advantage of being unconditionally stable and will yield physically accurate results over relatively large time increments. Thus, it is often utilized as a means of incorporating computational robustness. For best accuracy, the Crank-Nicholson scheme is the superior choice, provided the time increment remains relatively small.

Here, the discretization equations will be developed in the most general possible form with the weighting factor retained as an arbitrary parameter such that the time integration scheme may be selected as a matter of choice in the study. For practical use, it is advantageous to multiply the generalized discretization equation (48) by $\Delta \xi$ and regroup common terms:

$$
\begin{aligned}
& \left(\frac{\Delta \xi}{\Delta \tau}\right) u_{i}^{m+1}-\left(\frac{\Delta \xi}{\Delta \tau}\right) u_{i}^{m}=f\left(\frac{1}{\Delta \xi}+\frac{n}{2 \xi_{i}}\right) u_{i+1}^{m+1}+(1-f)\left(\frac{1}{\Delta \xi}+\frac{n}{2 \xi_{i}}\right) u_{i+1}^{m} \\
& +f\left(\frac{1}{\Delta \xi}-\frac{n}{2 \xi_{i}}\right) u_{i-1}^{m+1}+(1-f)\left(\frac{1}{\Delta \xi}-\frac{n}{2 \xi_{i}}\right) u_{i-1}^{m} \\
& -f\left(\frac{2}{\Delta \xi}\right) u_{i}^{m+1}-(1-f)\left(\frac{2}{\Delta \xi}\right) u_{i}^{m}+f S_{i}^{m+1} \Delta \xi+(1-f) S_{i}^{m} \Delta \xi
\end{aligned}
$$

or

$$
\begin{aligned}
{\left[\frac{\Delta \xi}{\Delta \tau}+\frac{2 f}{\Delta \xi}\right] u_{i}^{m+1} } & =f\left[\frac{1}{\Delta \xi}+\frac{n}{2 \xi_{i}}\right] u_{i+1}^{m+1}+f\left[\frac{1}{\Delta \xi}-\frac{n}{2 \xi_{i}}\right] u_{i-1}^{m+1} \\
& +\left[\frac{\Delta \xi}{\Delta \tau}-(1-f) \frac{2}{\Delta \xi}\right] u_{i}^{m}+(1-f)\left[\frac{1}{\Delta \xi}+\frac{n}{2 \xi_{i}}\right] u_{i+1}^{m} \\
& +(1-f)\left[\frac{1}{\Delta \xi}-\frac{n}{2 \xi_{i}}\right] u_{i-1}^{m}+f S_{i}^{m+1} \Delta \xi+(1-f) S_{i}^{m} \Delta \xi
\end{aligned}
$$


This result applies to all interior nodes $(i=2, \ldots, N-1)$.

3.1.1.2. Boundary Nodes. Closure of the computational domain requires specification of boundary conditions at the symmetry axis and at the external surface $\partial \Omega$ and construction of discretization equations valid at the corresponding boundary nodes.

The development for the symmetry boundary condition, $\partial u /\left.\partial \xi\right|_{\xi=0}=0$, is straightforward and directly follows from a forward difference approximation,

$$
\left.\frac{\partial u}{\partial \xi}\right|_{\xi=0} \approx \frac{u_{2}-u_{1}}{\Delta \xi}+o(\Delta \xi)=0,
$$

from which we deduce the desired discretization equation at the symmetry node $(i=1)$,

$$
u_{1}^{m+1}=u_{2}^{m+1}
$$

As a first step in the development of a discretization equation for the surface boundary node, consider a 3-node support centered at $i=N$ where a fictitious nodal value $u^{*}$ has been introduced as illustrated in figure 12. Then, introduction of the central difference approximation for the derivative in the boundary condition, equation (45), gives the expression:

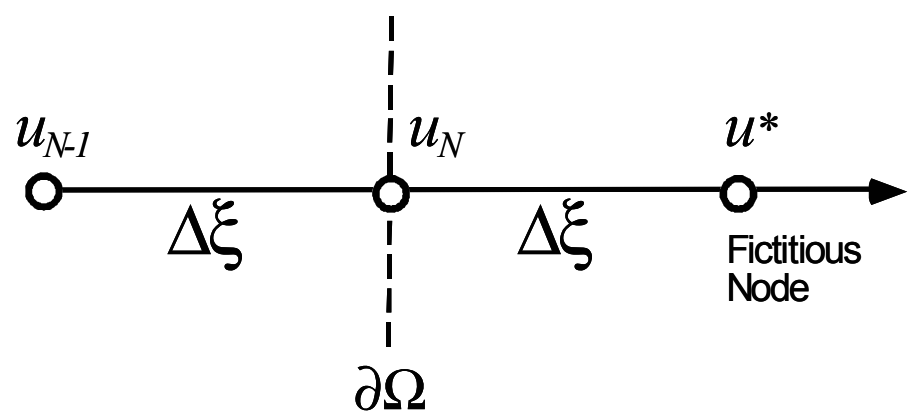

Figure 12. Computational grid structure at external boundary, including fictitious node.

$$
\left.\frac{\partial u}{\partial \xi}\right|_{\xi=1} \approx \frac{u^{*}-u_{N-1}}{2 \Delta \xi}=B i\left(U-u_{N}\right),
$$

which may be solved for the fictitious dimensionless temperature $u^{*}$ :

$$
u^{*}=2 \Delta \xi B i\left(U-u_{N}\right)+u_{N-1}
$$

Forming the central difference approximation for the second spatial derivative at the surface boundary yields the result

$$
\left.\frac{\partial^{2} u}{\partial \xi^{2}}\right|_{\xi=1} \approx \frac{u^{*}+u_{N-1}-2 u_{N}}{\Delta \xi^{2}}
$$


and elimination of $u^{*}$ using equation (54) provides the relationship

$$
\left.\frac{\partial^{2} u}{\partial \xi^{2}}\right|_{\xi=1} \approx \frac{2 \Delta \xi B i U-2(1+\Delta \xi B i) u_{N}-2 u_{N-1}}{\Delta \xi^{2}}
$$

Now, we allow $U$ to be a time dependent variable, introduce equations (53) and (56) into the reaction-diffusion equation (44), and discretize over a small time increment $\Delta \tau$ to produce a generalized discretization equation centered on the surface boundary node $N$ :

$$
\begin{aligned}
\frac{u_{N}^{m+1}-u_{N}^{m}}{\Delta \tau} & =f\left[\frac{2 \Delta \xi B i U^{m+1}-2(1+\Delta \xi B i) u_{N}^{m+1}+2 u_{N-1}^{m+1}}{\Delta \xi^{2}}\right]+f\left(\frac{n B i}{\xi_{N}}\right)\left(U^{m+1}-u_{N}^{m+1}\right) \\
& +\frac{f}{2} S_{N}^{m+1}+(1-f)\left[\frac{2 \Delta \xi B i U^{m}-2(1+\Delta \xi B i) u_{N}^{m}+2 u_{N-1}^{m}}{\Delta \xi^{2}}\right] \\
& +(1-f)\left(\frac{n B i}{\xi_{N}}\right)\left(U^{m}-u_{N}^{m}\right)+\frac{(1-f)}{2} S_{N}^{m} .
\end{aligned}
$$

Note in this case that the source term has been halved, since the boundary cell volume external to the computational domain is half that of an interior cell. For practical purposes, we multiply equation (57) through by $\Delta \xi$ and regroup common terms to obtain:

$$
\begin{aligned}
\left(\frac{\Delta \xi}{\Delta \tau}\right) u_{N}^{m+1} & -\left(\frac{\Delta \xi}{\Delta \tau}\right) u_{N}^{m}=f\left[2 B i+\frac{n B i \Delta \xi}{\xi_{N}}\right] U^{m+1}+(1-f)\left[2 B i+\frac{n B i \Delta \xi}{\xi_{N}}\right] U^{m} \\
& +\mathrm{f}\left(\frac{2}{\Delta \xi}\right) u_{N-1}^{m+1}+(1-f)\left(\frac{2}{\Delta \xi}\right) u_{N-1}^{m}-f\left[\frac{2(1+\Delta \xi B i)}{\Delta \xi}+\frac{n B i \Delta \xi}{\xi_{N}}\right] u_{N}^{m+1} \\
& -(1-f)\left[\frac{2(1+\Delta \xi B i)}{\Delta \xi}+\frac{n B i \Delta \xi}{\xi_{N}}\right] u_{N}^{m}+f \frac{\Delta \xi}{2} S_{N}^{m+1}+(1-f) \frac{\Delta \xi}{2} S_{N}^{m}
\end{aligned}
$$

or

$$
\begin{aligned}
{\left[\frac{\Delta \xi}{\Delta \tau}+f\left(\frac{1+\Delta \xi B i}{\Delta \xi / 2}+\frac{n \Delta \xi B i}{\xi_{N}}\right)\right] u_{N}^{m+1}=\left(\frac{2}{\Delta \xi}\right) u_{N-1}^{m+1} } \\
+\left[\frac{\Delta \xi}{\Delta \tau}-(1-f)\left(\frac{1+\Delta \xi B i}{\Delta \xi / 2}+\frac{n \Delta \xi B i}{\xi_{N}}\right)\right] u_{N}^{m} \\
+\left[2 B i+\frac{n \Delta \xi B i}{\xi_{N}}\right]\left[f U^{m+1}+(1-f) U^{m}\right]+f \frac{\Delta \xi}{2} S_{N}^{m+1}+(1-f) \frac{\Delta \xi}{2} S_{N}^{m} .
\end{aligned}
$$


3.1.1.3 Source Term Linearization. The objective of the discretization procedure is to reduce the partial differential equation problem to a set of linear algebraic equations, which may be effectively attacked using powerful linear algebra techniques. Thus, an additional linearization approximation must be introduced for the Arrhenius source term in the preceding development, since it contains a nonlinear exponential function, $S=\lambda e^{-1 / u}$.

The simplest approach in this case is to treat the source term in a fully explicit manner such that

$$
S_{i}^{m+1}=S_{i}^{m} \quad ; \quad i=1, \ldots, N
$$

Utilization of this approximation is straightforward and requires no further explanation.

An alternative and somewhat more sophisticated method relies on an expansion of the form

$$
\frac{d S}{d \tau}=\frac{d S}{d u} \frac{d u}{d \tau}
$$

which may be temporally discretized to obtain a linearized representation for the source term at grid point $i$ :

$$
S_{i}^{m+1}=S_{i}^{m}+\left.\frac{d S}{d u}\right|_{i} ^{m}\left(u_{i}^{m+1}-u_{i}^{m}\right) .
$$

Taking the indicated derivative generates the following linear algebraic expression in terms of $u_{i}^{m+1}$ :

$$
S_{i}^{m+1}=S_{i}^{m}\left(1-\frac{1}{u_{i}^{m}}\right)+\left[\frac{S_{i}^{m}}{\left(u_{i}^{m}\right)^{2}}\right] u_{i}^{m+1} .
$$

Substitution of this expression into the previously developed discretization equations (50) and (59) yields the following forms:

$$
\begin{aligned}
{\left[\frac{\Delta \xi}{\Delta \tau}+\frac{2 f}{\Delta \xi}\right.} & \left.-\frac{f S_{i}^{m} \Delta \xi}{\left(u_{i}^{m}\right)}\right] u_{N}^{m+1}=f\left[\frac{1}{\Delta \xi}+\frac{n}{2 \xi_{i}}\right] u_{i+1}^{m+1}+f\left[\frac{1}{\Delta \xi}-\frac{n}{2 \xi_{i}}\right] u_{i-1}^{m+1} \\
+ & {\left[\frac{\Delta \xi}{\Delta \tau}-(1-f) \frac{2}{\Delta \xi}\right] u_{i}^{m}+(1-f)\left[\frac{1}{\Delta \xi}+\frac{n}{2 \xi_{i}}\right] u_{i+1}^{m} } \\
+ & (1-f)\left[\frac{1}{\Delta \xi}-\frac{n}{2 \xi_{i}}\right] u_{i-1}^{m}\left(1-\frac{f}{u_{i}^{m}}\right) S_{i}^{m} \Delta \xi \quad ; i=2, \ldots, N-1 .
\end{aligned}
$$




$$
\begin{aligned}
{\left[\frac{\Delta \xi}{\Delta \tau}+f\right.} & \left.\left.\frac{1+\Delta \xi B i}{\Delta \xi / 2}+\frac{n \Delta \xi B i}{\xi_{N}}-f\left(\frac{\Delta \xi}{2}\right) \frac{S_{N}^{m}}{\left(u_{N}^{m}\right)^{2}}\right)\right] u_{N}^{m+1}=\left(\frac{2}{\Delta \xi}\right) u_{N-1}^{m+1} \\
& +\left[\frac{\Delta \xi}{\Delta \tau}-(1-f)\left(\frac{1+\Delta \xi B i}{\Delta \xi / 2}+\frac{n \Delta \xi B i}{\xi_{N}}\right)\right] u_{N}^{m} \\
& +\left[2 B i+\frac{n \Delta \xi B i}{\xi_{N}}\right]\left[f U^{m+1}+(1-f) U^{m}\right]+\left(1-\frac{f}{u_{N}^{m}}\right) \frac{\Delta \xi}{2} S_{N}^{m} \quad ; i=N .
\end{aligned}
$$

Discretization equation (52) at the symmetry node $(i=1)$ is unaffected by this linearization procedure and requires no modification.

\subsubsection{Solution Algorithm}

Now that the numerical solution has been reduced to a system of linear algebraic equations, we find it advantageous to adopt a linear vector space notation. Application of the preceding discretization equations at each nodal point, for instance, yields a system of equations that may be compactly expressed using the matrix equation

$$
K U^{m+1}+L U^{m}+M=0,
$$

where $K, L$, and $M$ are $N \times N$ square matrices containing numerical coefficients and $U$ is an $N$-element column vector containing the dependent variable values at the grid points. As before, superscripts denote temporal indices. The general solution of equation (66) at time step $m+1$ follows immediately and has the basic form:

$$
U^{m+1}=K^{-1}\left[L U^{m}+M\right],
$$

where $K^{-1}$ is the matrix inverse of $K$.

Equation (67) may be solved by a number of algorithms, but the most convenient and simple follows from the standard Gaussian elimination procedure. Because the non-zero elements of $K$ align themselves along the central three diagonals of the matrix, the elimination process turns into a particularly simple recurrence sequence. This method is commonly referred to as the TDMA (Tri-Diagonal Matrix Algorithm). ${ }^{30}$ form

To implement TDMA, we first proceed by writing the generalized discretization equation in the

$$
a_{i} u_{i}^{m+1}=b_{i} u_{i+1}^{m+1}+c_{i} u_{i-1}^{m+1}+d_{i},
$$


where

$$
\begin{aligned}
& a_{i}= \begin{cases}\frac{\Delta \xi}{\Delta \tau}+\frac{2 f}{\Delta \xi}-f \Delta \xi \frac{S_{i}^{m}}{\left(u_{i}^{m}\right)^{2}} & i=1 \\
\frac{\Delta \xi}{\Delta \tau}+f\left[\frac{1+\Delta \xi B i}{\Delta \xi / 2}+\frac{n \Delta \xi B i}{\xi_{N}}\right]-f\left(\frac{\Delta \xi}{2}\right) \frac{S_{N}^{m}}{\left(u_{N}^{m}\right)^{2}} & i=2, \ldots, N-1 \\
& i=N\end{cases}
\end{aligned}
$$

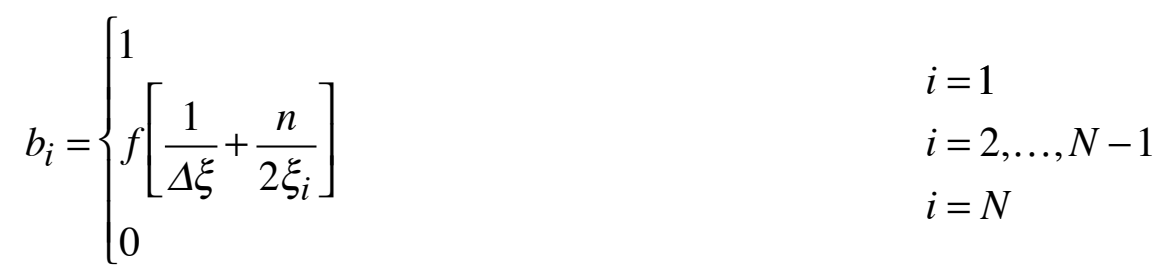

$$
\begin{aligned}
& c_{i}= \begin{cases}1 & \\
f\left[\frac{1}{\Delta \xi}-\frac{n}{2 \xi_{i}}\right] & i=1 \\
2 / \Delta \xi & i=2, \ldots, N-1 \\
& i=N\end{cases} \\
& i=1 \\
& i=2, \ldots, N-1 \\
& i=N
\end{aligned}
$$


Formal derivation of TDMA may be found in standard textbooks on numerical analysis (see ref. 30, for instance). The algorithm procedures are summarized as follows:

- $\quad$ Start the recurrence process by calculating the parameters.

$$
P_{1}=\frac{b_{1}}{a_{1}} \quad Q_{1}=\frac{d_{1}}{a_{1}} .
$$

- $\quad$ Continue the TDMA recurrence sequence over the range $i=2,3, \ldots, N$ using the relations

$$
\begin{aligned}
P_{i} & =\frac{b_{i}}{a_{i}-c_{i} P_{i-1}} \\
Q_{i} & =\frac{d_{i}+c_{i} Q_{i-1}}{a_{i}-c_{i} P_{i-1}} .
\end{aligned}
$$

- At the other end of the sequence, note that $b_{N}=0$, which leads to $P_{N}=0$. Hence,

$$
u_{N}^{m+1}=Q_{N}
$$

- $\quad$ The remaining unknown grid values may then be determined by marching backward $(i=$ $N-1, \ldots, 1)$ using the back substitution relation

$$
u_{i}^{m+1}=P_{i} u_{i+1}^{m+1}+Q_{i}
$$

\subsection{Validation}

The numerical methodology for nonstationary thermal ignition was implemented in FORTRAN programming language to enable automated machine processing on a digital computer platform. As a validation measure, comparative baseline calculations were then performed with respect to published solutions in the peer-reviewed literature. For simplicity, these calculations were all performed assuming an initially uniform temperature distribution within the reactive material as defined by the relation

$$
u(\xi, 0)=U+C
$$

where $U$ is the dimensionless ambient temperature and $C$ is an arbitrary constant representing an initial perturbative displacement from thermal equilibrium.

Given values of $\lambda, U$, and $B i$, our principal interest becomes a determination of the critical value for $C$, denoted by $C_{c r}$, a threshold value which distinguishes between those initial conditions that converge to an upper steady state branch associated with ignition and those that converge to a non-igniting stable lower branch. Validation of the nonstationary numerical model is established through direct comparison of predicted values for $C_{c r}$ with the published results of Weber et al. ${ }^{4}$

Validation calculations were performed for all three principal centrosymmetric solids using CrankNicholson time integration $(f=0.5)$ with the controlling physical parameters defined as $\lambda=10^{6}, U=0.05$, 
and $B i \rightarrow \infty$. Critical initial threshold temperatures were determined through trial and error, and representative transients for the dimensionless centerline temperature are shown in figures 13, 14, and 15 for $n=0$, $n=1$, and $n=2$, respectively. Note that the centerline temperature directly corresponds to the maximum value in the material due to the imposed physical symmetry for the problem.

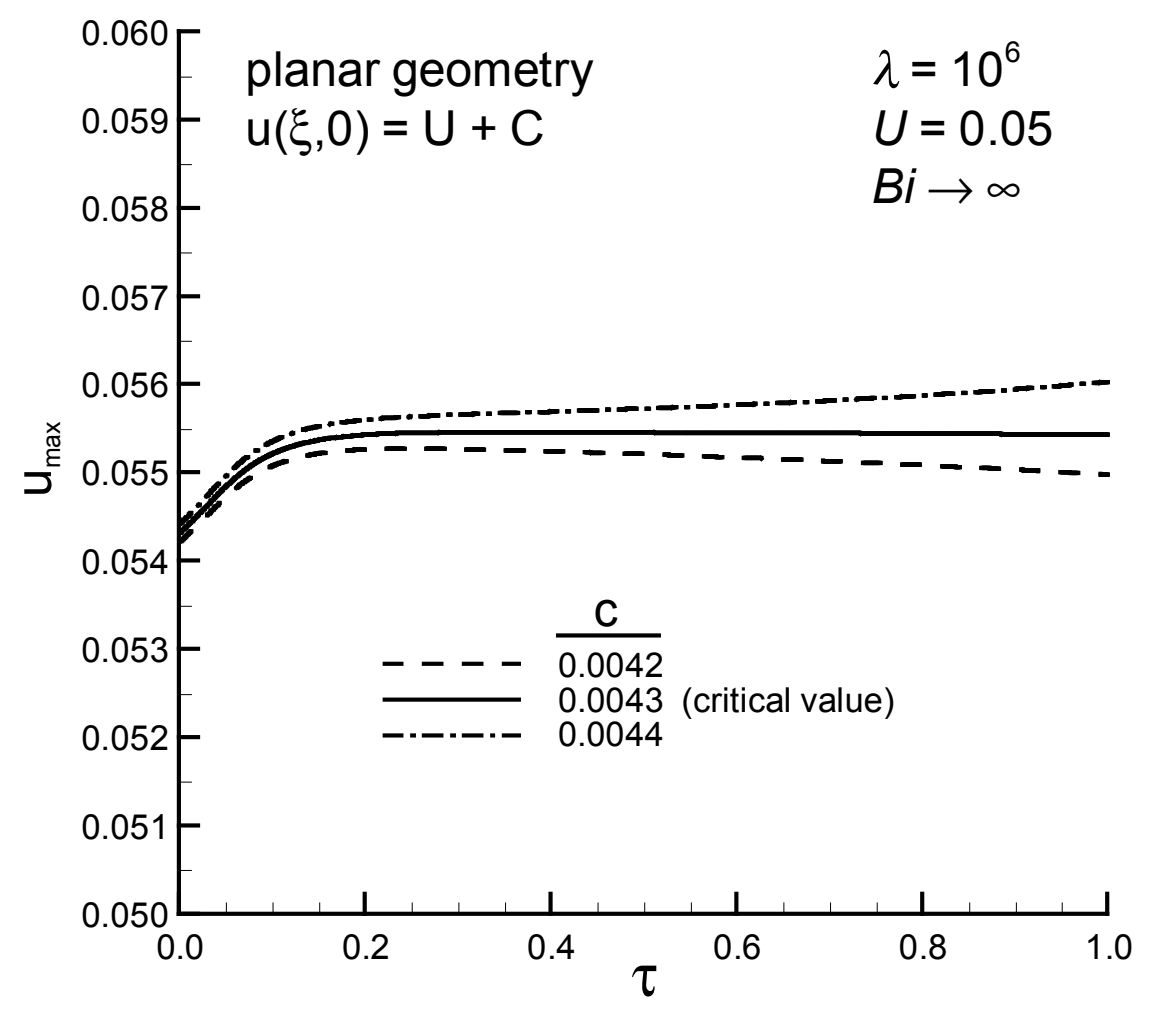

Figure 13. Centerline temperature transients for $n=0\left(\lambda=10^{6}\right.$ and $\left.B i \rightarrow \infty\right)$.

Numerically predicted threshold values for ignition were $C_{c r}=0.0043, C_{c r}=0.0083$, and $C_{c r}=0.0101$ for the planar, cylindrical, and spherical geometries, all of which are in exact agreement with the published results of Weber et al. ${ }^{4}$ The stable upper steady state branches are not displayed in these figures since it is several orders of magnitude larger than the lower steady state branches. It should be pointed out in passing, moreover, that the upper branch serves only as a mathematical attractor and has no practical physical interpretation, since ignition would occur long before the upper branch could be attained. For fundamental demonstration purposes, an upper steady state branch for an igniting spherical geometry case $(C=0.011)$ is shown in figure 16. The ignition delay, the period of time required for the initial condition to evolve to the upper steady state branch, may be directly inferred from these types of graphs.

The numerical methodology was also evaluated for finite surface convective heat transfer rates to validate proper response to arbitrary environmental boundary conditions. These calculations were performed for the planar slab geometry using Crank-Nicholson time integration $(f=0.5)$ with the controlling physical parameters defined as $\lambda=10^{6}$ and $U=0.03$. The resulting variation in $C_{c r}$ as a function of Biot 


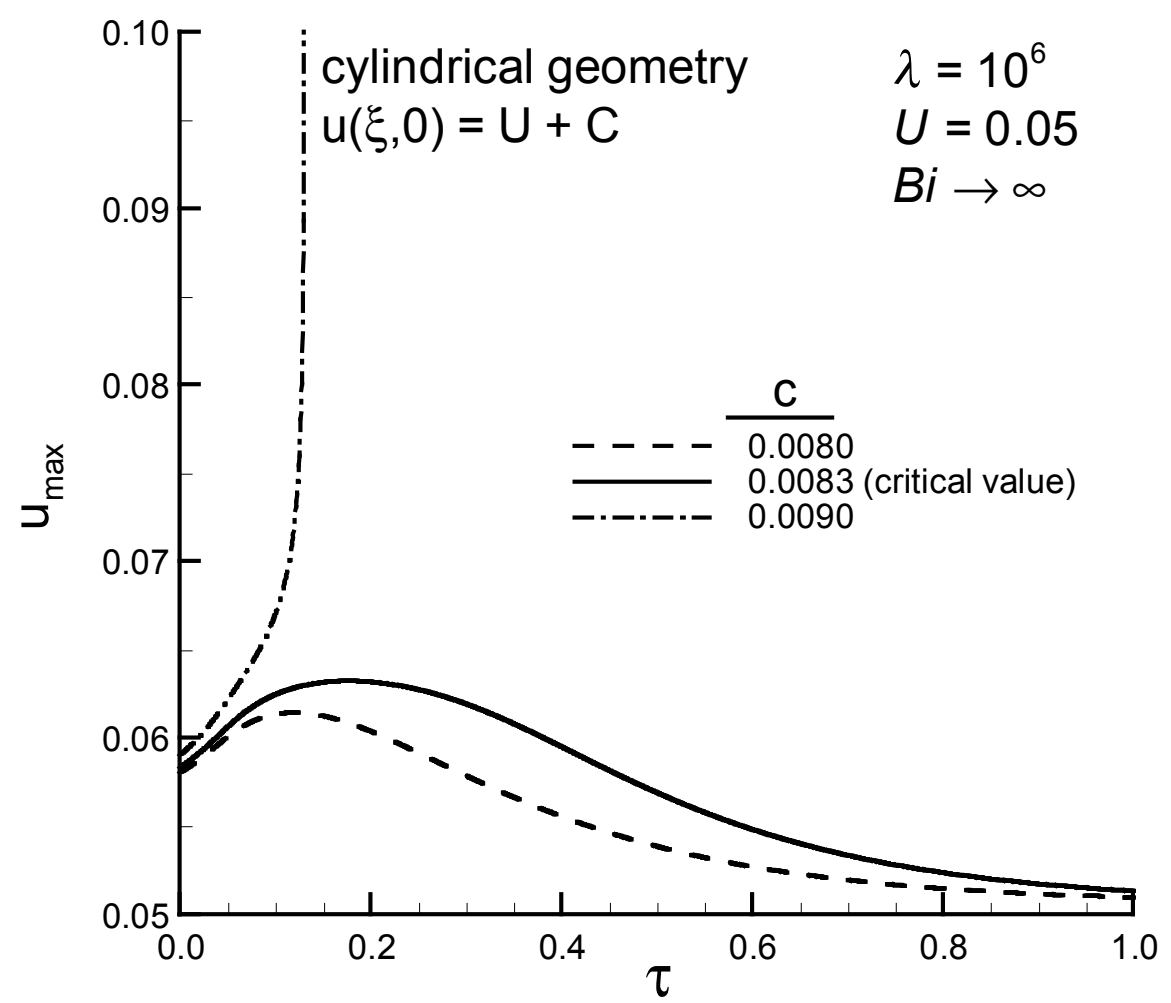

Figure 14. Centerline temperature transients for $n=1\left(\lambda=10^{6}\right.$ and $\left.B i \rightarrow \infty\right)$.

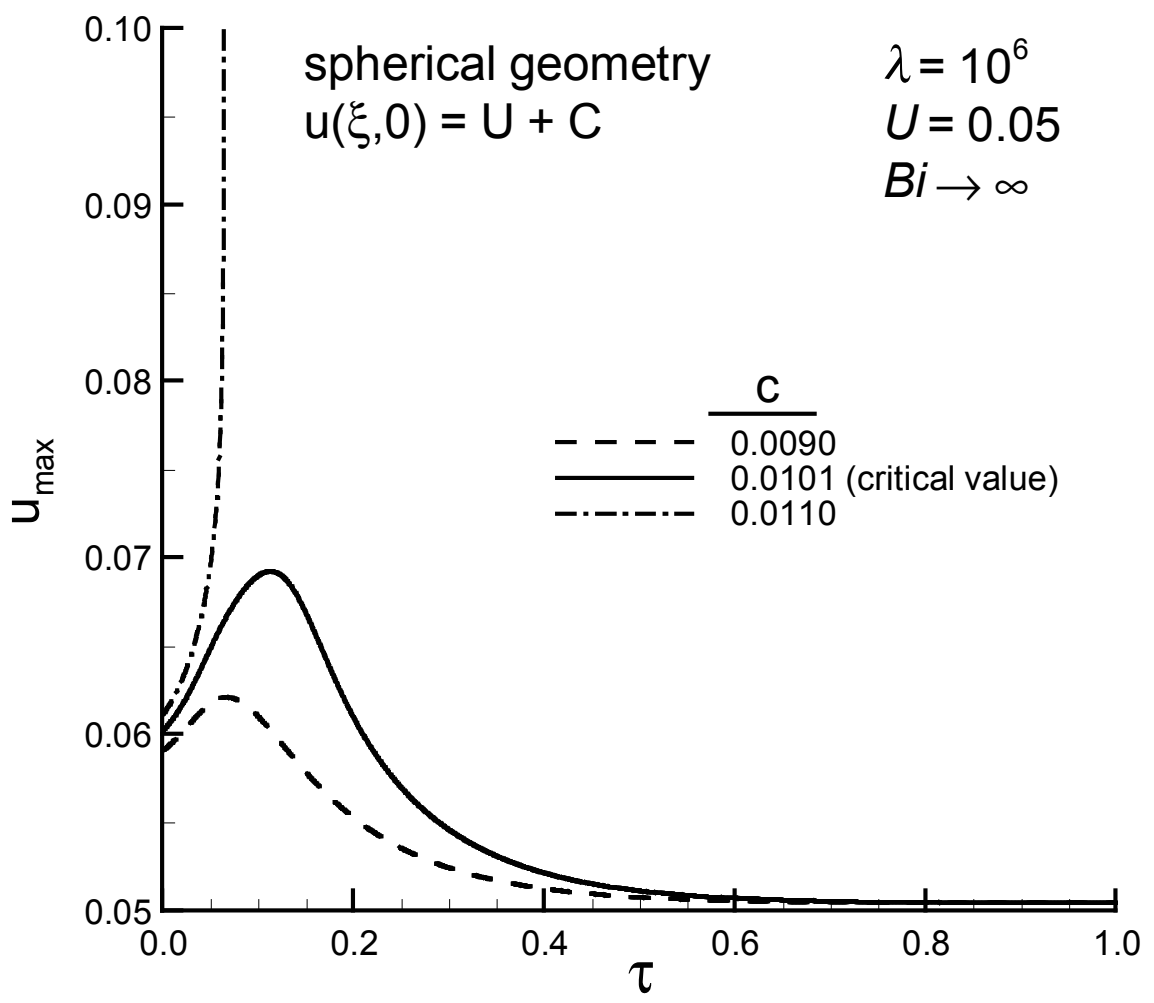

Figure 15. Centerline temperature transients for $n=2\left(\lambda=10^{6}\right.$ and $\left.B i \rightarrow \infty\right)$. 


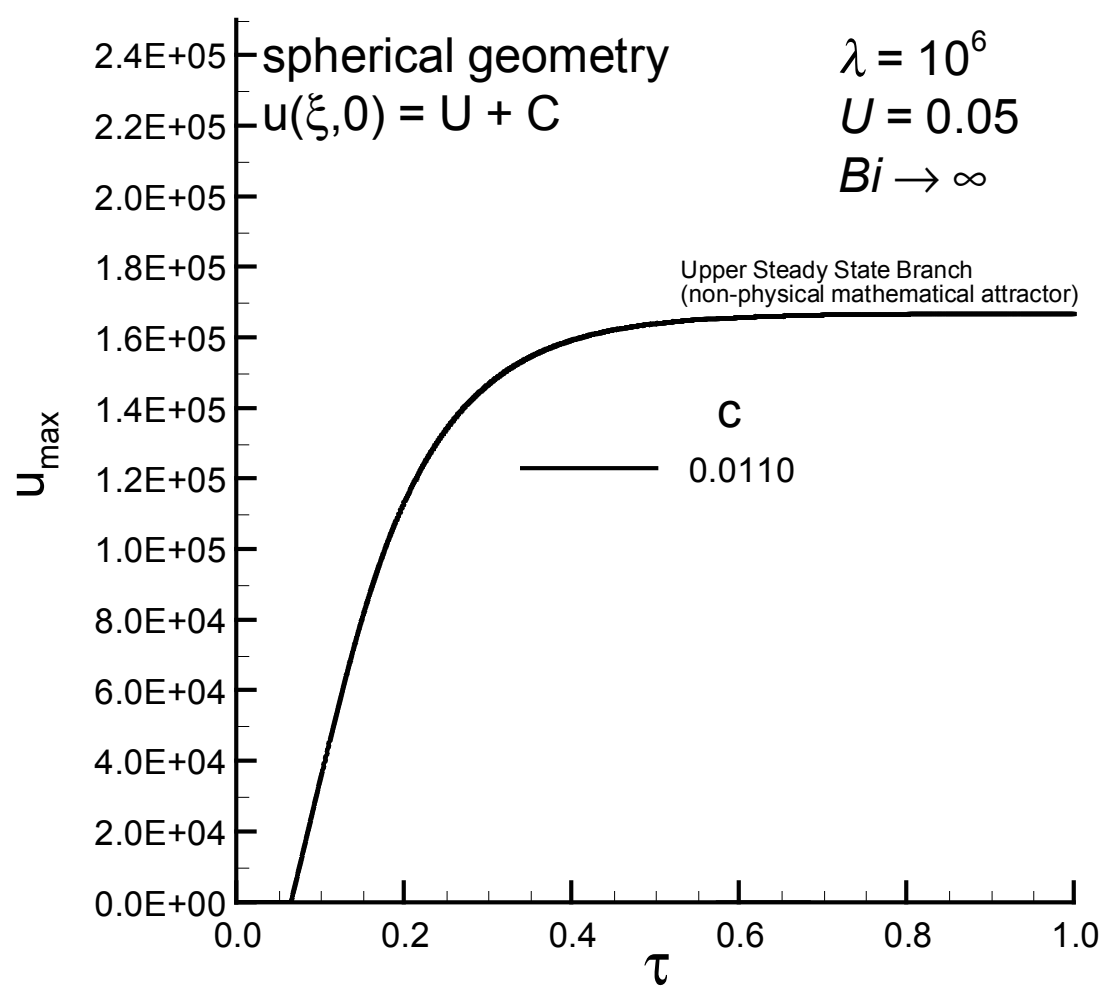

Figure 16. Upper steady state branch for $n=2\left(\lambda=10^{6}\right.$ and $\left.B i \rightarrow \infty\right)$.

number is shown in figure 17, with the exact numerical values tabulated as an inset to the graph. These results are also in excellent agreement with the published data of Weber et al., ${ }^{4}$ and demonstrate that the initial condition threshold for ignition becomes relatively insensitive to changes in $B i$ once it exceeds a value of about 5 .

As a final validation step, calculations were performed for a practical case involving the self-heating of aerated milk powder, which is frequently stored in an ambient temperature environment immediately following a high-temperature drying procedure. This particular case, based on an actual self-heating fire in New Zealand, ${ }^{31}$ was also analyzed by Weber et al., and their results are again used as a comparative baseline. ${ }^{4}$ The characteristic physical parameters for milk powder are well established, and the planar slab geometry was considered an adequate approximation to the actual physical conditions associated with the incident. The established dimensionless parameters for the thermal ignition model are $\lambda=2.88 \times 10^{12}$, given a half-width of $0.2 \mathrm{~m}$, and $\lambda=11.5 \times 10^{12}$, given a half-width of $0.4 \mathrm{~m}$, with $U=0.0241$. Representative transients for the dimensionless centerline temperature are shown in figures 18 and 19 yielding the critical threshold values $C_{c r}=0.0058$ and $C_{c r}=0.0044$ for slab depths of $0.2 \mathrm{~m}$ and $0.4 \mathrm{~m}$, respectively. These results are again in excellent agreement with published results. ${ }^{4}$

Based on the demonstrated agreement and excellent concordance with established results for a variety of problem conditions, it was concluded that the proposed numerical methodology had been tested and validated to a satisfactory extent. Although not discussed in specific detail, the methodology was also thoroughly exercised with both explicit and implicit source term linearization schemes, and the resulting 


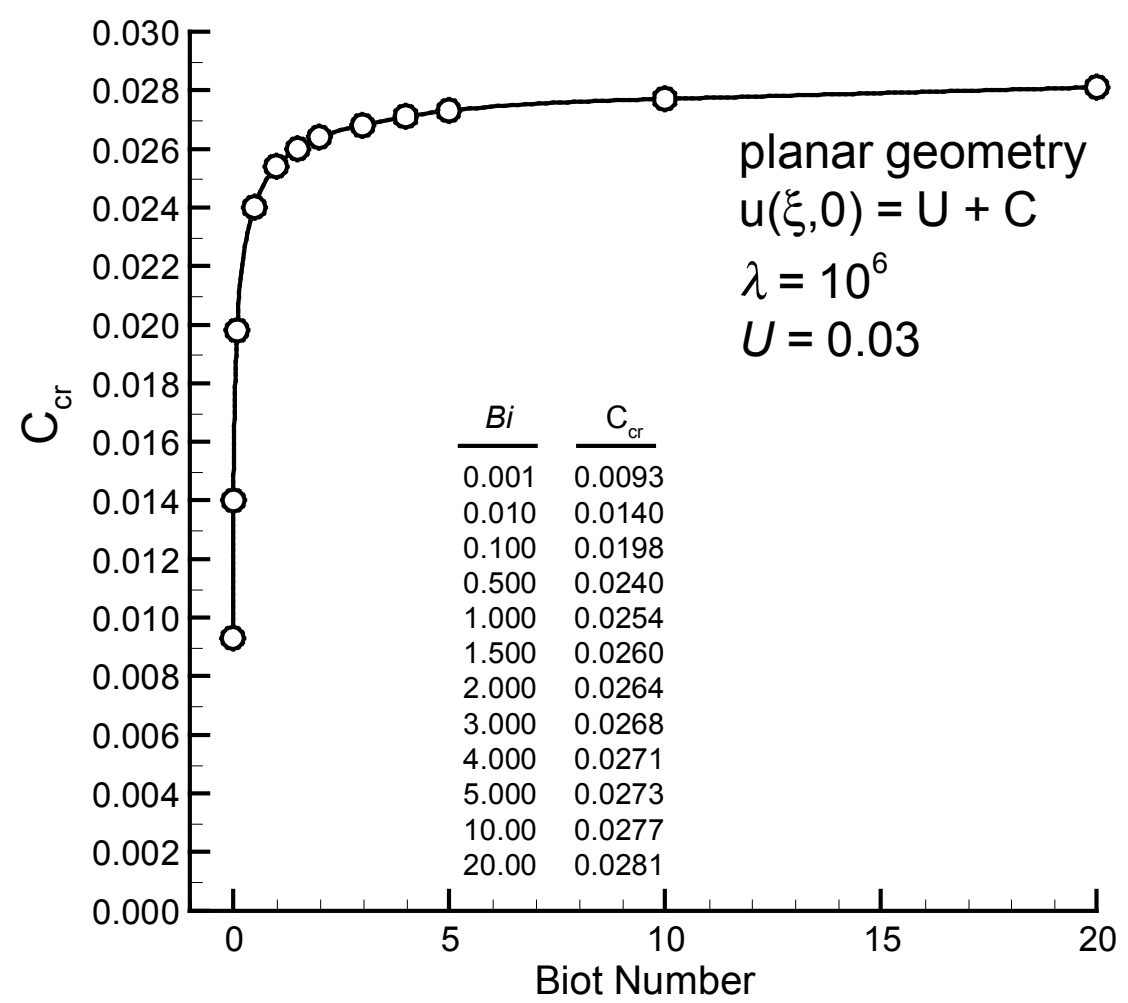

Figure 17. Variation in $C_{c r}$ as a function of $B i$ with $\lambda=10^{6}$ and $U=0.03$.

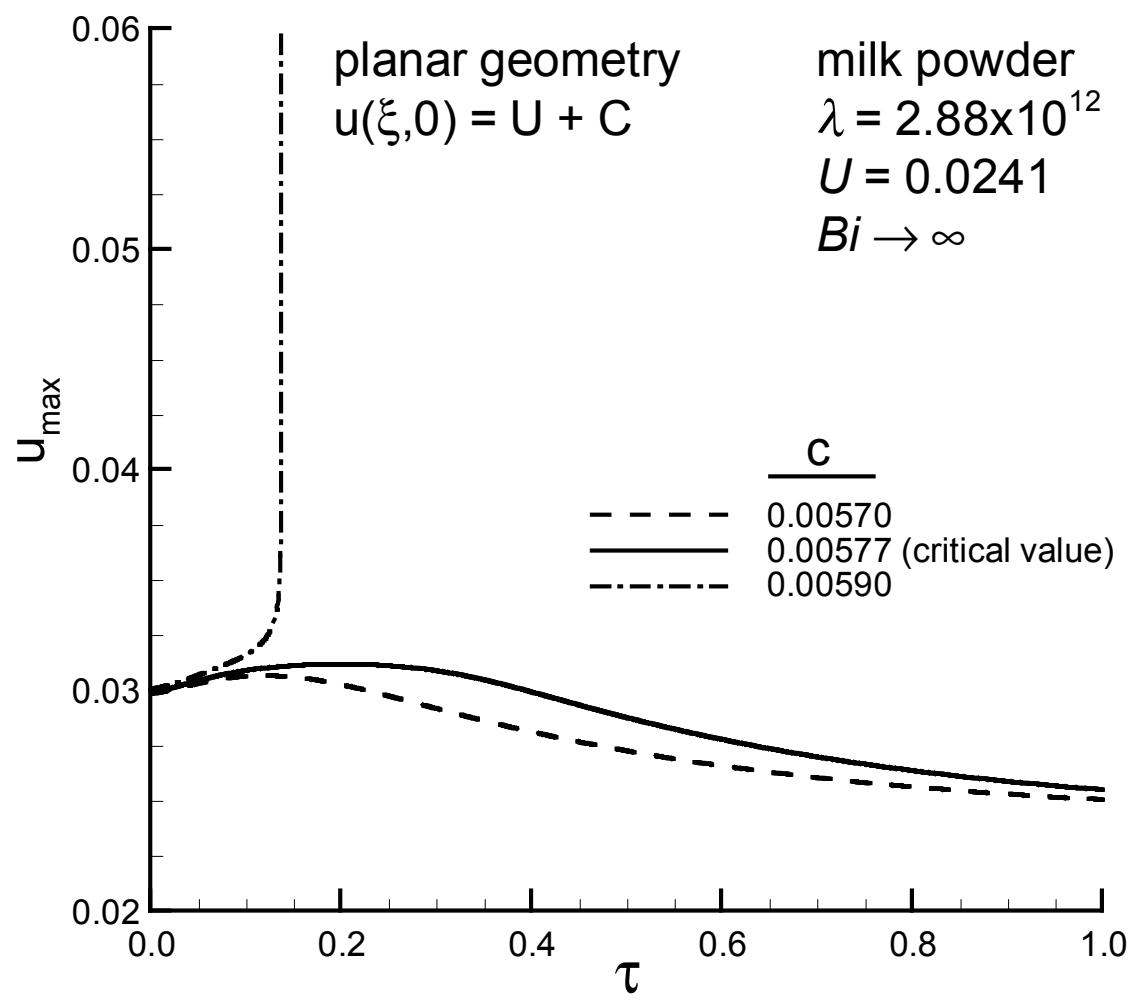

Figure 18. Centerline temperature transients for milk powder $\left(\lambda=2.88 \times 10^{12}\right)$. 


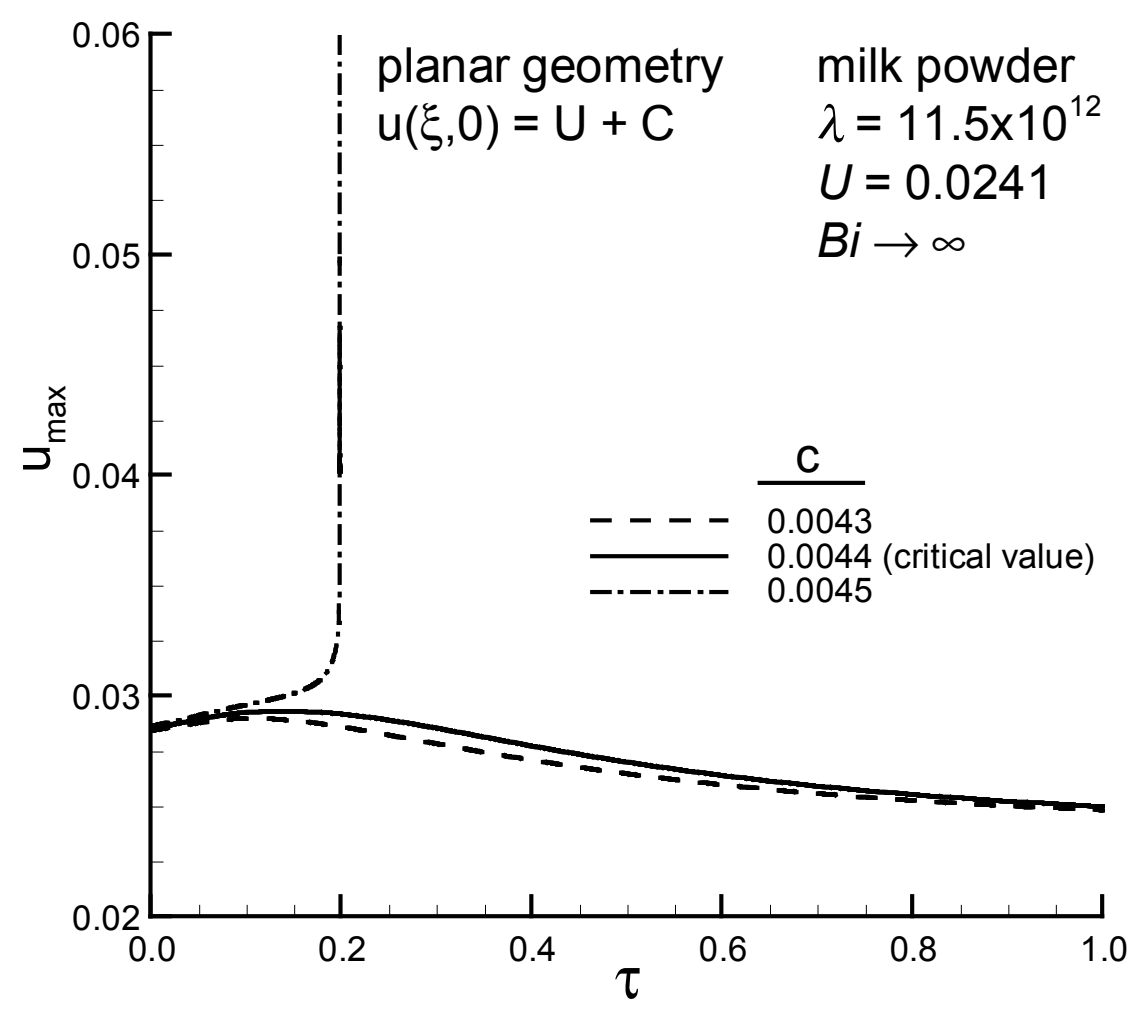

Figure 19. Centerline temperature transients for milk powder $\left(\lambda=11.5 \times 10^{12}\right)$.

numerical predictions were found to be completely insensitive to the linearization method being utilized. On the basis of these extensive validation efforts, it was concluded that the numerical methodology established in this study could serve as a reliable and dependable tool for undertaking a thorough study of the critical initial conditions for thermal ignition. 


\section{ASSEMBLY PROBLEM AND CRITICAL INITIAL CONDITIONS}

With the establishment of validated stationary (steady-state) and nonstationary (transient) models for thermal ignition/explosion, it becomes possible to undertake a judicious examination of the so-called 'assembly' problem (see section 1.2), in which the fate of a self-heating material can be strongly dependent on initial conditions. Relevant physical examples include the safety of processed reactive materials when placed into piles or bins while hot and the initiation of explosives by localized hot spots. From a practical perspective, it is extremely important to know the models' respective ranges of applicability for arbitrary circumstances of assembly; therefore, a deeper understanding of the underlying mathematical structure is highly desirable.

The central issue at hand is whether the initial temperature excess in the assembly will continue to rise indefinitely or dissipate through conductive transport and Newtonian cooling to the surroundings. Of particular interest to this study is the critical demarcation boundary between those initial conditions serving as an onset to ignition/explosion and those which ultimately decay to a self-extinguishing quench state. For the general case of a non-uniform assembly, the transient solution for the evolved state may be expected to display a marked sensitivity to the spatial concentration of thermal energy at the outset, and criticality will depend on the initial temperature distribution. In this section, we examine such subtle mathematical issues in detail by incorporating a generalized self-consistent treatment of the initial temperature distribution for the non-stationary model, which permits direct comparison of criticality predictions against the stationary model.

The purpose is to precisely determine the conditions under which use of the steady-state modeling approach is legitimate or illegitimate, and to establish deeper insight into the structure of the solution space. It should be noted as a warning, however, that reactants are consumed in all real systems and no true steady states are physically possible. As such, the temperature rise accompanying self-heating can only exhibit a maximum before ultimately decaying to ambient as the exothermic reaction approaches completion and is overtaken by cooling, even after large increases associated with explosive effects, and it is no longer possible to recognize ignition/explosion as a fundamental discontinuity. Here, we have followed conventional practice and neglected reactant consumption by reasoning that if the heat of reaction is high enough, concentration changes preceding the onset of a large and sudden temperature rise, corresponding to explosion or ignition, will be negligibly small. The validity of conclusions drawn from this study is therefore limited by the validity of this basic underlying assumption.

\subsection{Initial Shape Profile}

As part of the previous validation exercises in section 3, we adopted the following convenient expression for an initially uniform temperature distribution within the material assembly:

$$
u(\xi, 0)=U+C
$$


where $U$ is the dimensionless ambient temperature and $C$ is an arbitrary constant representing the initial perturbative displacement from ambient conditions. The principal objective in that case, given values for $U, \lambda$, and $B i$, was determination of the critical value for $C$, denoted by $C_{c r}$, which defines the watershed threshold between those initial conditions that evolve to an upper steady-state (i.e., ignition/explosion) and those that evolve to a self-extinguishing steady-state quench.

We now extend this basic approach by introducing a one-parameter shape profile $g(\xi)$ to define a generalized nonuniform initial temperature distribution having the form

$$
u(\xi, 0)=U+C g(\xi)
$$

Again, we seek critical values, $C_{c r}$, such that any initial conditions with $C<C_{c r}$ evolve to the lower (cool) steady state and any conditions with $C>C_{c r}$ evolve to the upper (hot) steady state. The initial shape profile is defined by the one-parameter function:

$$
\begin{array}{lrl}
g(\xi) & =A_{\varepsilon}\left(1-\xi^{\varepsilon}\right), & \varepsilon \geq 0 \\
g(\xi) \rightarrow 1, & \varepsilon \rightarrow \infty,
\end{array}
$$

where $\varepsilon$ is the initial profile shape factor and $A_{\varepsilon}$ is a normalization factor for preserving total heat content irrespective of profile shape. With this definition, we anticipate the existence of a family of critical initial condition profiles for each value of the initial shape factor, $\varepsilon$.

Normalization is achieved through a constraint on the spatial integral of $g(\xi)$ such that it is invariant with $\varepsilon$. The value of this integral is taken to be unity for convenience:

$$
I=\int_{0}^{1} g(\xi) d \xi=1
$$

Thus, substitution of equation (80) and execution of elementary integration steps yield the following expression for the normalization factor:

$$
A_{\varepsilon}=\frac{\varepsilon+1}{\varepsilon}
$$

which clearly demonstrates that $A_{\varepsilon}$ must decrease as $\varepsilon$ increases in order to preserve energy content in the distribution. Normalized initial shape profiles for representative values of the shape factor are shown in figure 20. These include linear $(\varepsilon=1)$, parabolic $(\varepsilon=2)$, and uniform $(\varepsilon=\infty)$ initial temperature distributions for the three principal centrosymmetric solids of interest to this study. The corresponding normalization factors are summarized in table 4.

\subsection{Criticality in Nonuniform Assemblies}

Our principal concern is to extend the scope of analysis to nonuniform assemblies and determine the variation in $C_{c r}$ with $U$, provided there are fixed values of $\lambda$ and $B i$, using $\varepsilon$ as an independent parameter for initial heat concentration. We may reasonably anticipate from physical intuition the following 


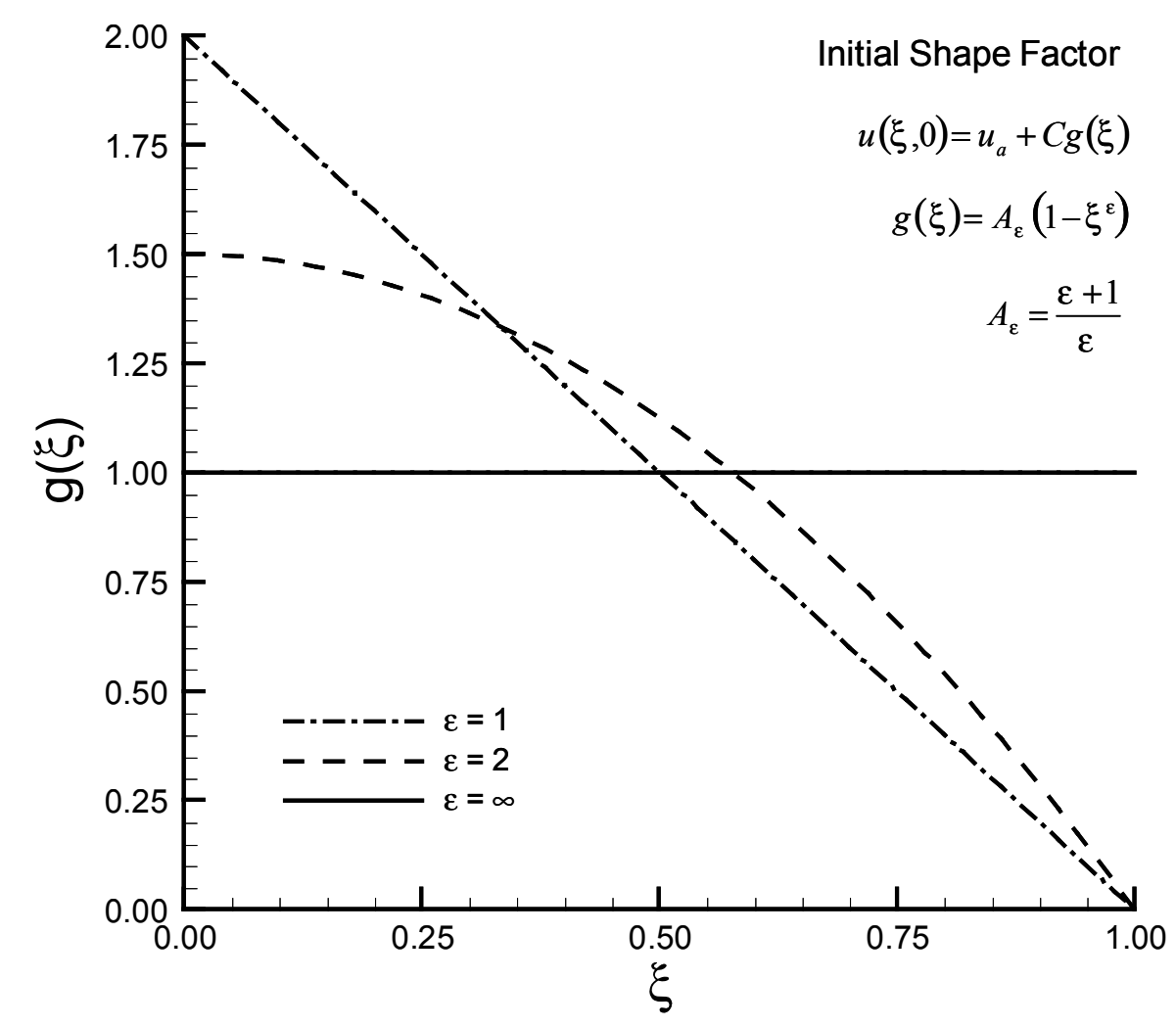

Figure 20. Normalized initial shape profiles for representative values of $\varepsilon$.

Table 4. Summary of profile shape factors and normalization factors.

\begin{tabular}{|c|c|}
\hline Profile Shape Factor $(\boldsymbol{\varepsilon})$ & Normalization Factor $\left(\boldsymbol{A}_{\boldsymbol{\varepsilon}}\right)$ \\
\hline 1 (linear) & 2 \\
2 (parabolic) & $3 / 2$ \\
$\infty$ (uniform) & 1 \\
\hline
\end{tabular}

functional dependence: (1) $C_{c r}$ will decrease as $U$ increases; (2) $C_{c r}$ will decrease as $\lambda$ increases; and (3) $C_{c r}$ will decrease as $B i$ decreases. It is intuitively obvious, however, that $A_{\varepsilon} C_{c r}$ would better serve as the parameter of choice in defining the critical threshold, since $A_{\varepsilon}$ exhibits a strong dependence on the value of $\varepsilon$. Previous cursory numerical investigation has supported the basic preceding conclusions, ${ }^{4}$ and the intent here is to buttress this argument with additional computational results. An additional outcome from the extended numerical analysis is improved understanding and deeper insight into the mathematical structure of the solution sets to the model problems.

To implement this numerical study, it was first necessary to construct an automated search procedure by which the nonstationary model could be used to determine $C_{c r}$ for arbitrary values of $\mathrm{U}$, given fixed values for $\lambda, B i$, and $\varepsilon$. This was accomplished in practice by assuming a very small value for $C$ and computing a fully evolved temporal solution for a selected value of $U$ using the coded numerical 
methodology for the nonstationary model. If the long-term solution is found to evolve to the lower steady state solution, the results are recorded, $C$ is given a small incremental increase, and the calculation is automatically repeated again and again. The value of $C_{c r}$ is ultimately resolved when a fully evolved solution to the upper steady state attractor is obtained. By starting with a very small value for $U$ and repeating the above procedure with incrementally increasing values, it is possible to fully construct the critical initial threshold curve out to the bifurcation point, $U_{c r}$.

The above automated search procedure was implemented in computer code, and calculations were then carried out for the three principal centrosymmetric solids given $\lambda=10^{4}, 10^{6}, 10^{8}$, assuming $B i \rightarrow \infty$. Although this crude search procedure proved effective and accurate for the purpose at hand, it is obviously inefficient and computationally expensive; the exact computing time being dependent, of course, on the size of the step increments and the speed of the microprocessor. In practice, it was necessary to balance the need for fidelity and resolution against the time required to construct a solution. Construction of a single threshold curve of satisfactory accuracy, for instance, would normally take several days of continuous run time on a dedicated personal computer.

Computational results for the nonuniform assembly problem are shown in figures 21-38, which summarize criticality characteristics for the planar slab, infinite cylinder, and sphere using linear $(\varepsilon=1)$, parabolic $(\varepsilon=2)$, and uniform $(\varepsilon=\infty)$ initial temperature distributions. For each value of $\lambda$ and geometry shape, the critical threshold curves of $u(0)=U+C_{c r} g(0)$ versus $U$ as well as the quench state curve $u(0)$ versus $U$ for each of the three simple geometric configurations under consideration. For purposes of comparison, stable lower and unstable intermediate branch solutions of the stationary model are also displayed on these graphs as dashed lines. The corresponding curves for $A_{\varepsilon} C_{c r}$ versus $U$ are also displayed since they will prove to be of significant importance in the analysis to follow.

The stable upper steady state curve is not shown since it is several orders of magnitude larger than the criticality and lower steady state branches and is of no physical consequence other than serving as a mathematical attractor for ignition.

The mathematical feature of essential importance and interest to be extracted from these results is the fact that the exact criticality threshold, as predicted by the nonstationary model, is significantly different from the unstable intermediate steady state, as predicted by the stationary model. Although the models yield identical predictions for the bifurcation point critical ambient temperature, $U_{c r}$, the ignition threshold for assembly of initially hot materials is found to be considerably lower than that deduced from the stationary model. This has serious implications since the widely held consensus view has always been that the unstable intermediate steady-state solution provides an adequate estimate for safe assembly of potentially hazardous self-heating materials. It is clear from these results, however, that this is a severely flawed postulate, the careless use of which could have grave practical consequences.

A particularly interesting point of additional note is the observation that the threshold temperature for ignition increases as $\varepsilon$ decreases and the initial heat distribution becomes more concentrated near the centerline. This seems somewhat counterintuitive, but can be explained from a heat balance perspective. That is, the higher temperature gradient associated with more concentrated heat deposition near the centerline increases outward heat conduction toward the surface boundary enough to outpace the self 


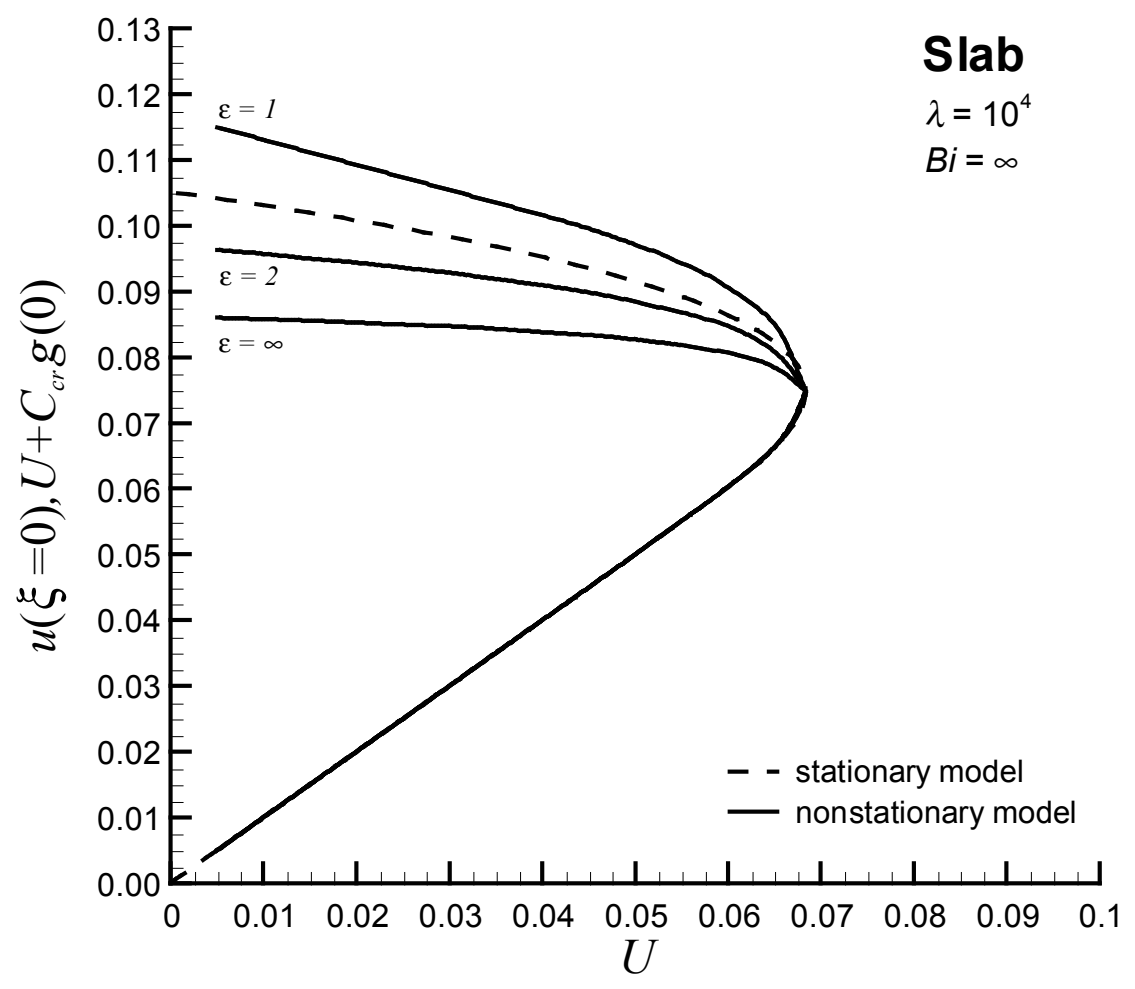

Figure 21. Criticality characteristics of nonuniform planar slab assemblies $\left(\lambda=10^{4}\right)$.

heating rate. It might be anticipated that this rate imbalance could be reversed given a sufficiently high exothermicity, but most practical substances of interest would not yield values very much beyond $\lambda=10^{8}$. Thus, a uniformly distributed assembly of hot material is potentially more dangerous than a nonuniform assembly with a hotter interior, given the same total heat content. The shape of the assembly also has a notable effect on ignitability in that the critical initial threshold temperature increases as we go from planar to cylindrical to spherical geometry. This is partly a surface-to-volume effect and is partly caused by the change in heat concentration.

Based on the preceding observations, one might speculate that the criticality threshold should somehow be correlated with the spatial moments of the initial heat distribution. The validity of this mathematical conjecture will be taken up in section 5 . 


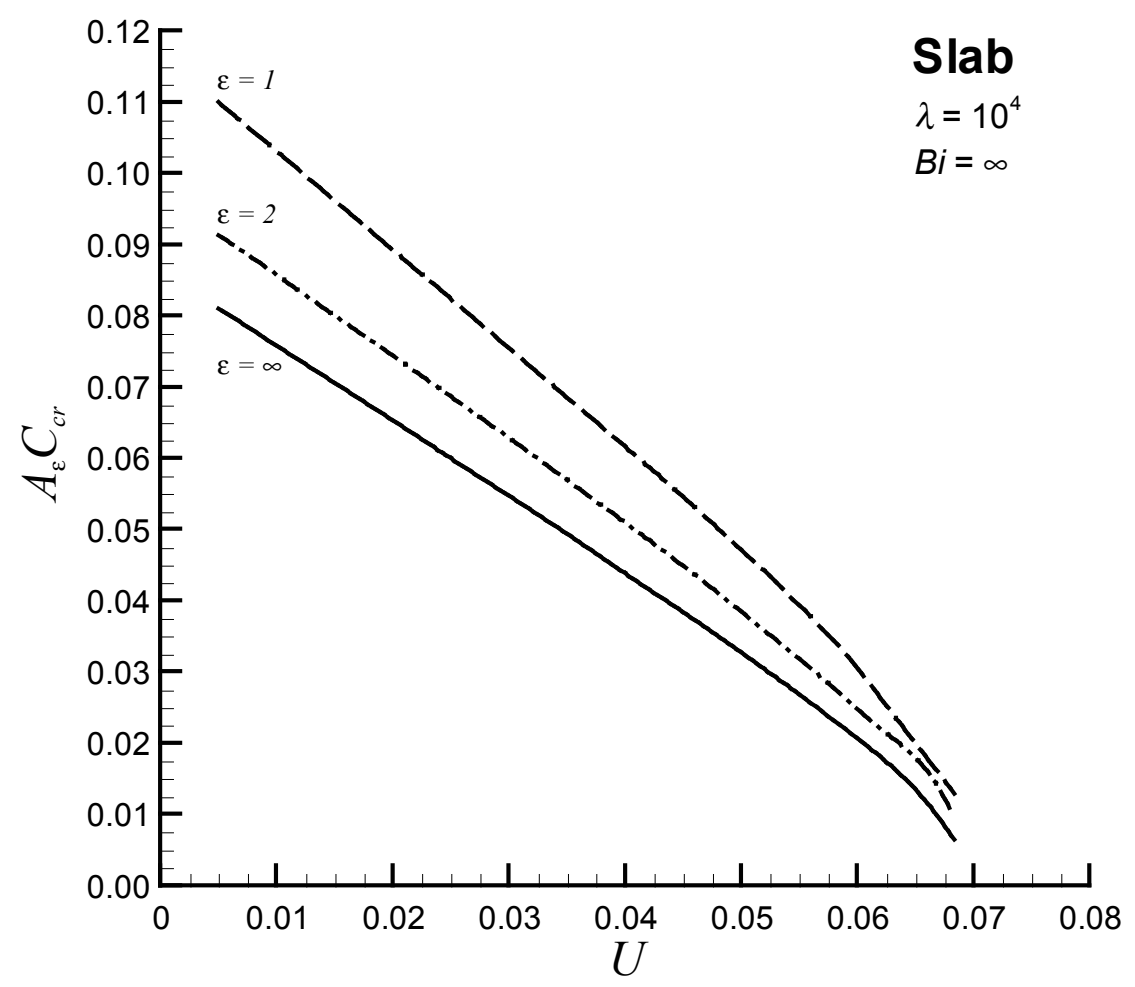

Figure 22. $A_{\varepsilon} C_{c r}$ versus $U$ curves for nonuniform planar slab assemblies $\left(\lambda=10^{4}\right)$.

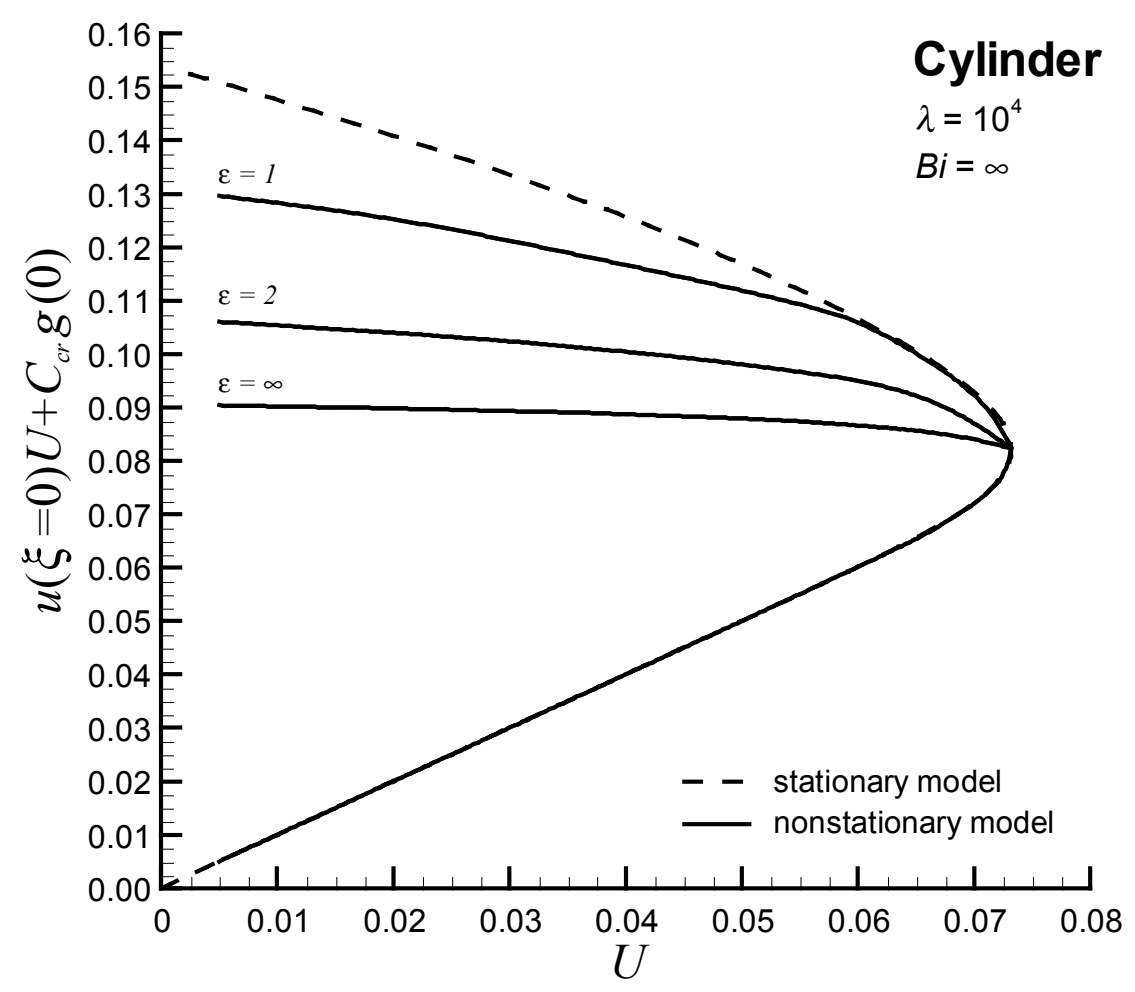

Figure 23. Criticality characteristics of nonuniform cylindrical assemblies $\left(\lambda=10^{4}\right)$. 


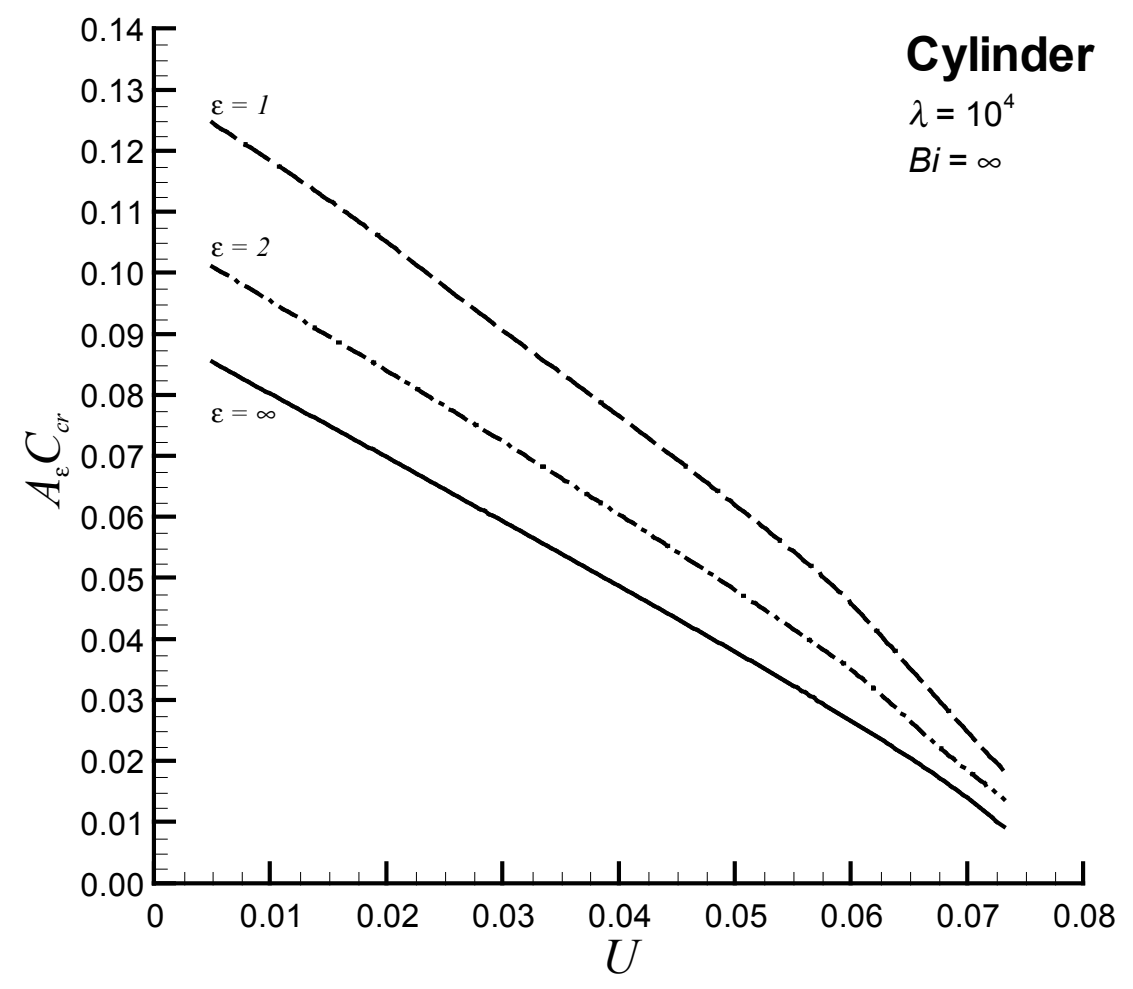

Figure 24. $A_{\varepsilon} C_{c r}$ versus $U$ curves for nonuniform cylindrical assemblies $\left(\lambda=10^{4}\right)$.

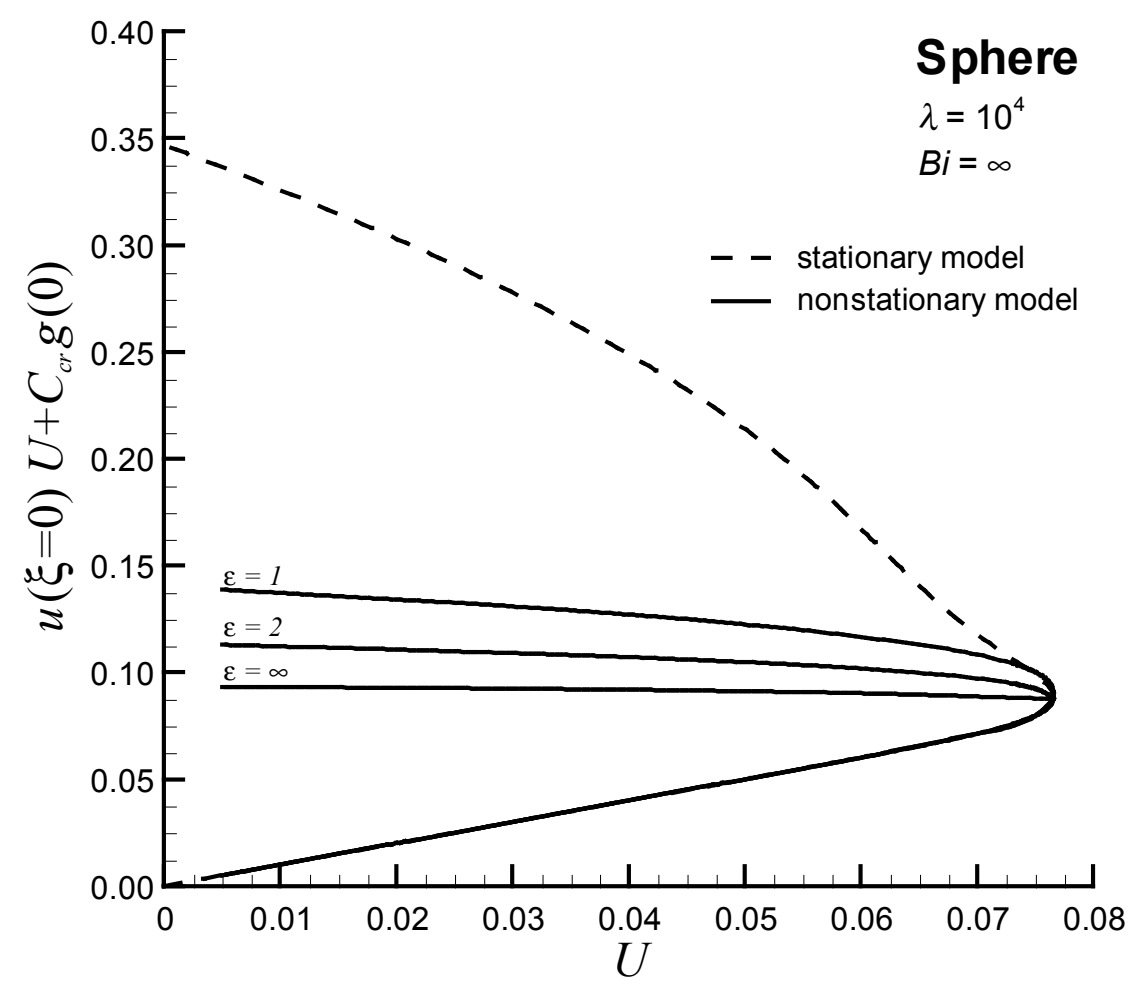

Figure 25. Criticality characteristics of nonuniform spherical assemblies $\left(\lambda=10^{4}\right)$. 


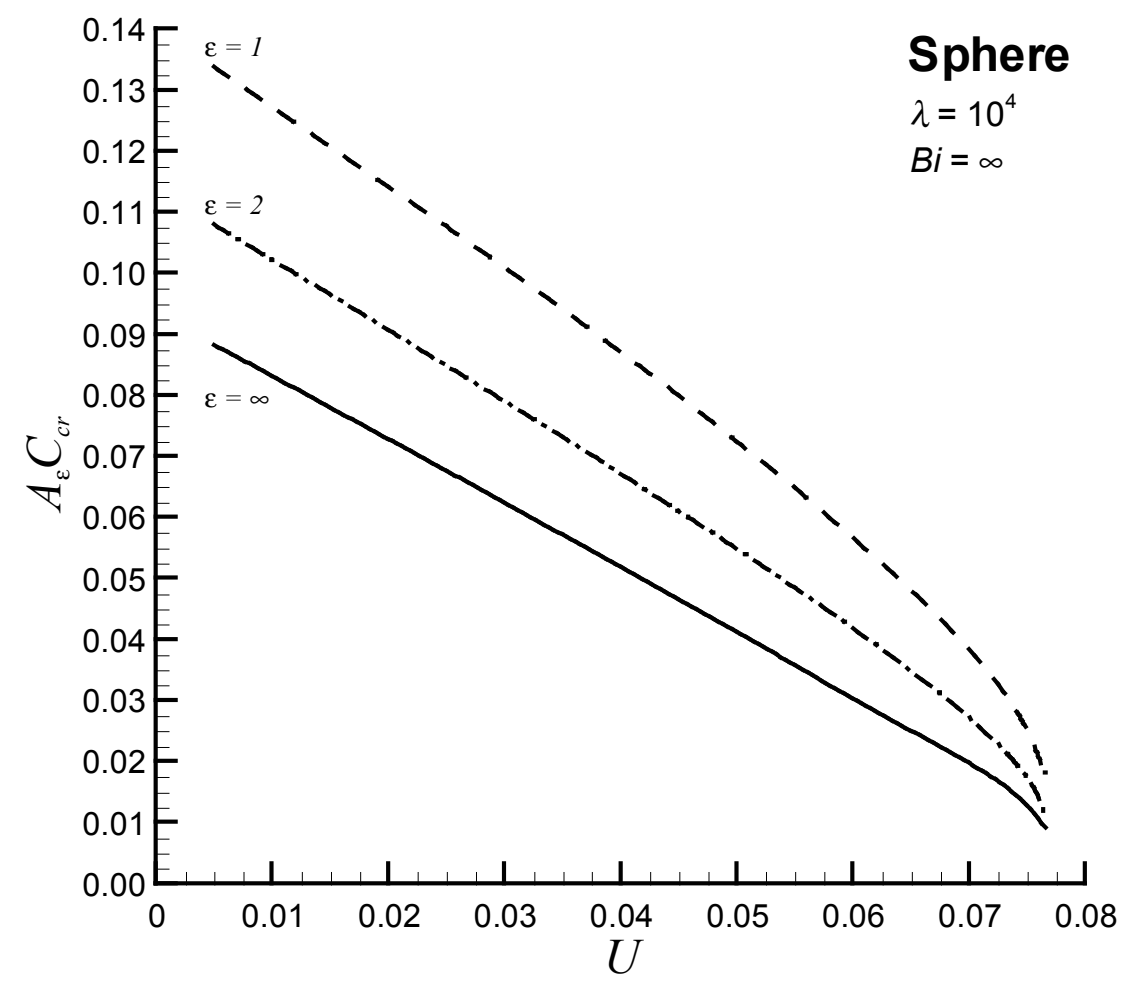

Figure 26. $A_{\varepsilon} C_{c r}$ versus $U$ curves for nonuniform spherical assemblies $\left(\lambda=10^{4}\right)$.

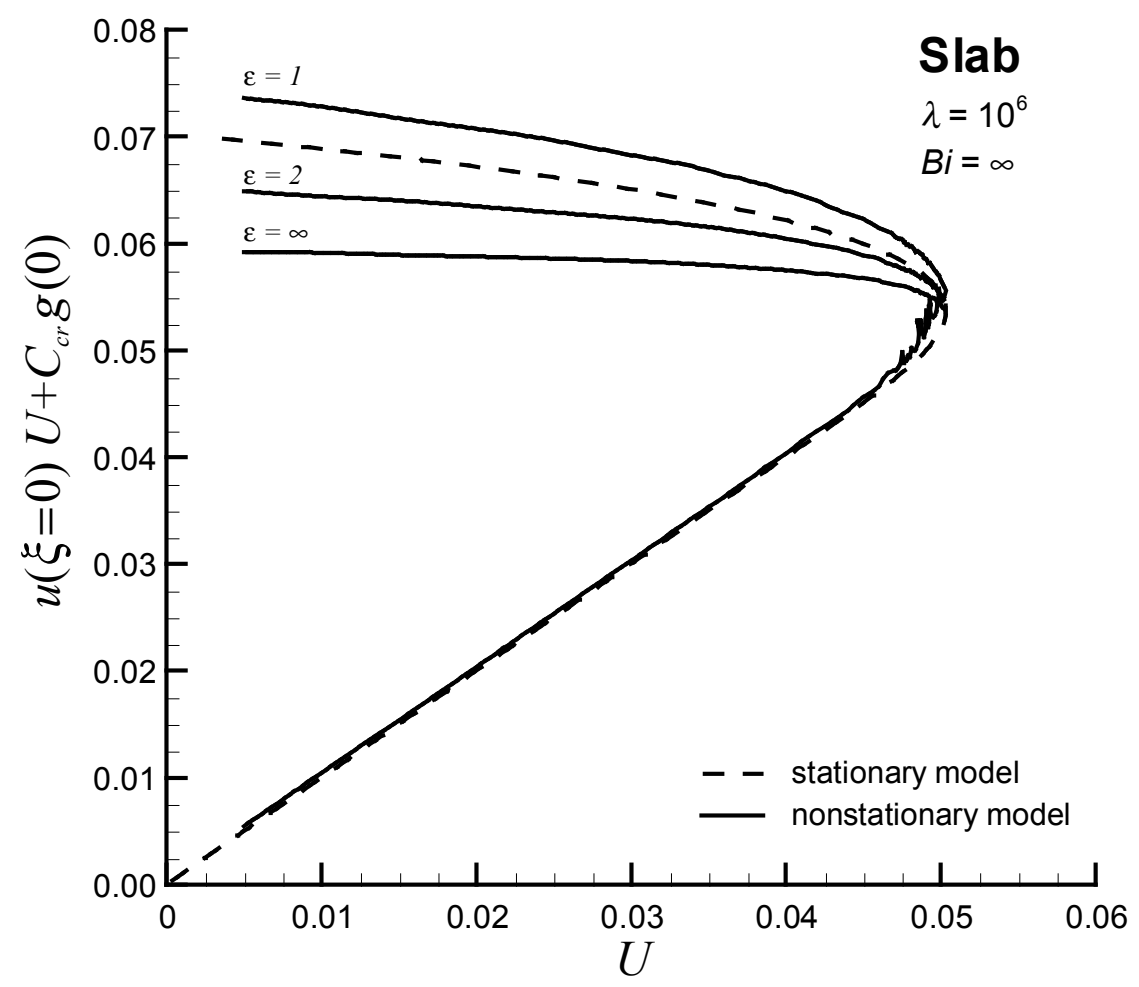

Figure 27. Criticality characteristics of nonuniform planar slab assemblies $\left(\lambda=10^{6}\right)$. 


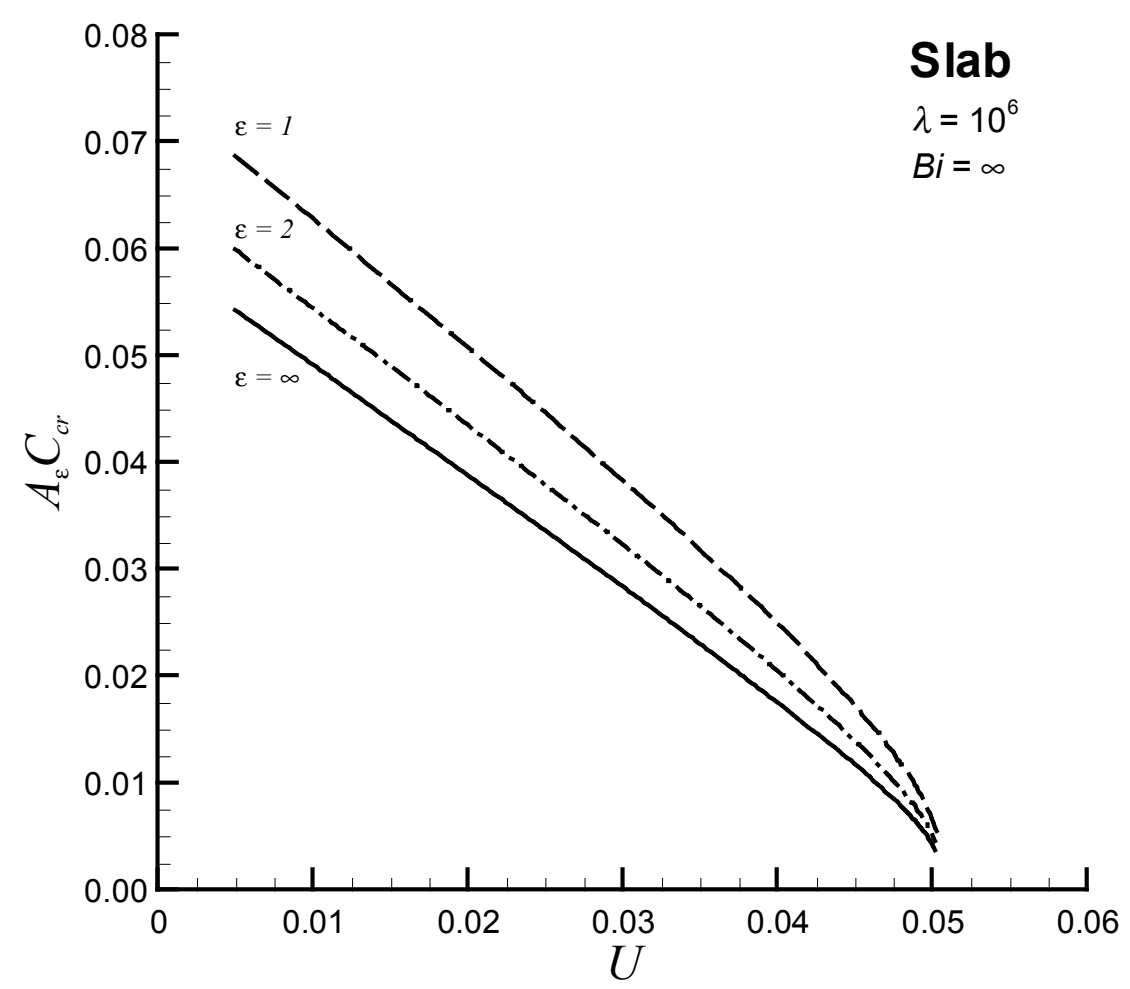

Figure 28. $A_{\varepsilon} C_{c r}$ versus $U$ curves for nonuniform planar slab assemblies $\left(\lambda=10^{6}\right)$.

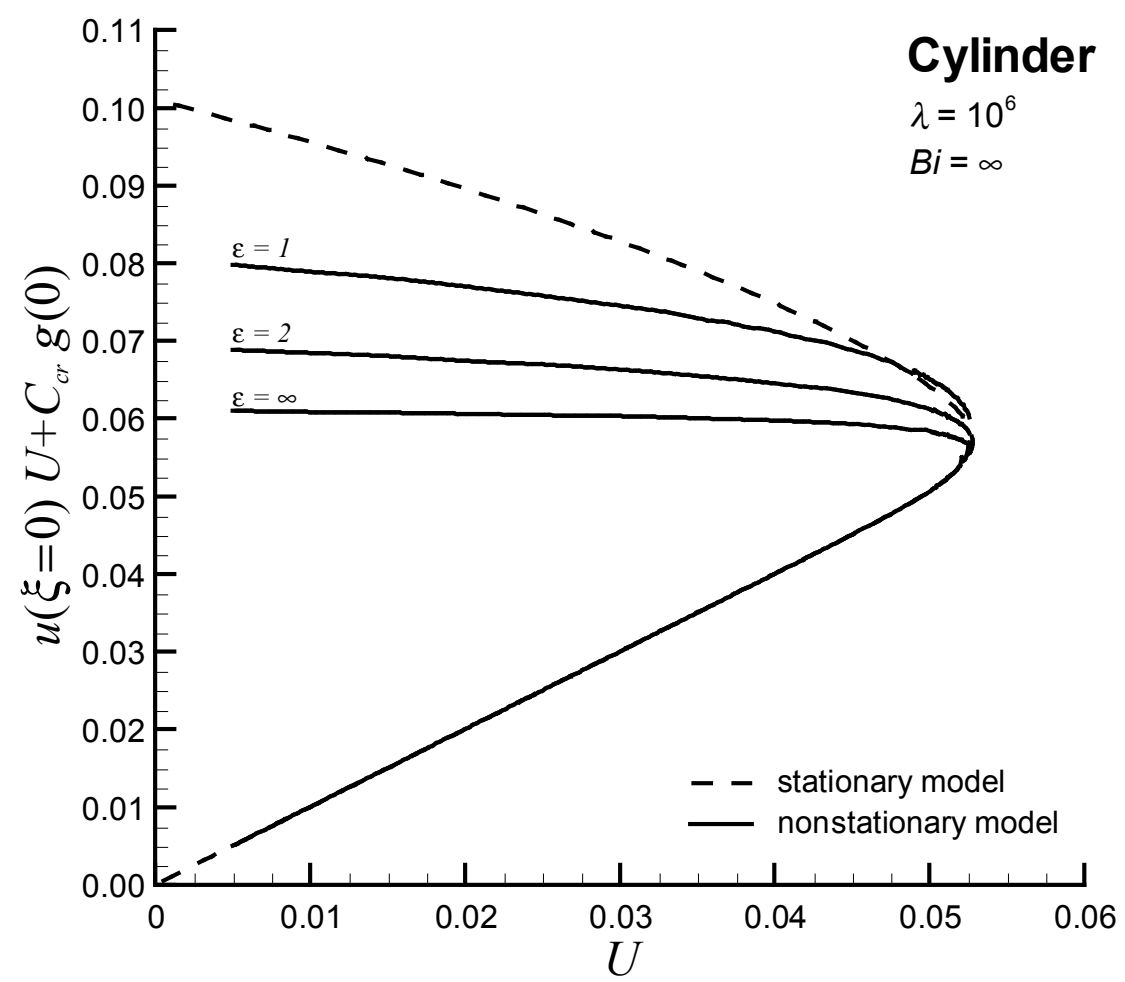

Figure 29. Criticality characteristics of nonuniform cylindrical assemblies $\left(\lambda=10^{6}\right)$. 


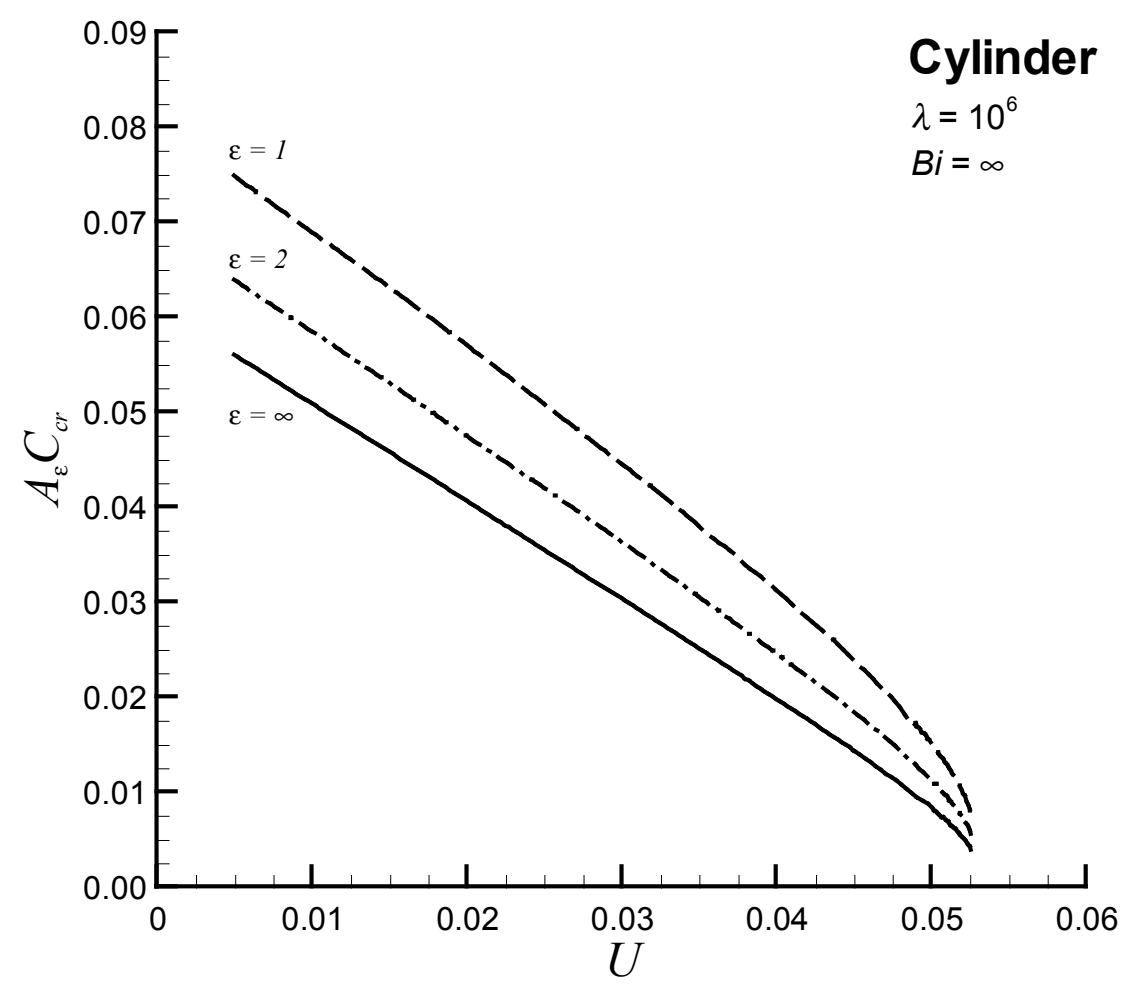

Figure 30. $A_{\varepsilon} C_{c r}$ versus $U$ curves for nonuniform cylindrical assemblies $\left(\lambda=10^{6}\right)$.

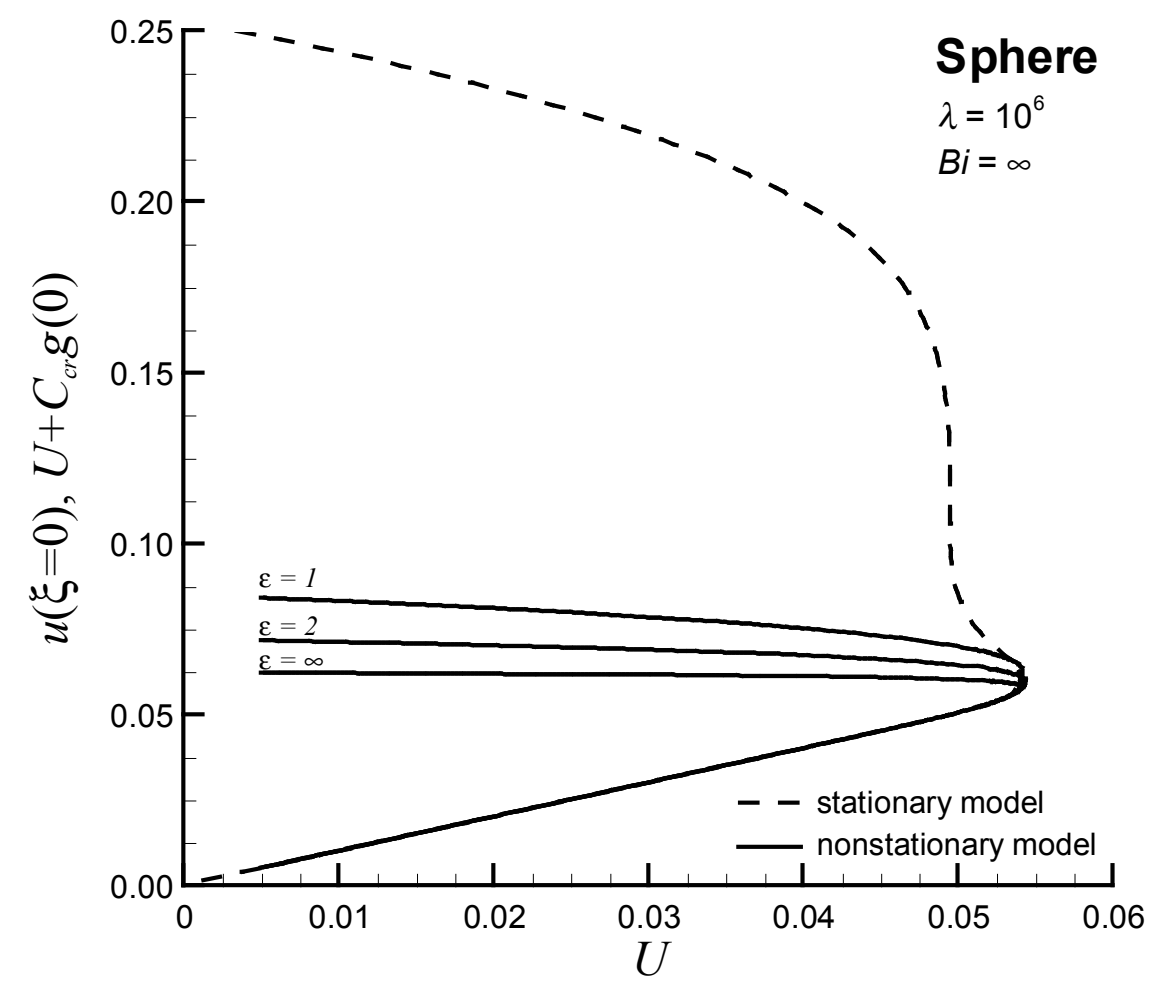

Figure 31. Criticality characteristics of nonuniform spherical assemblies $\left(\lambda=10^{6}\right)$. 


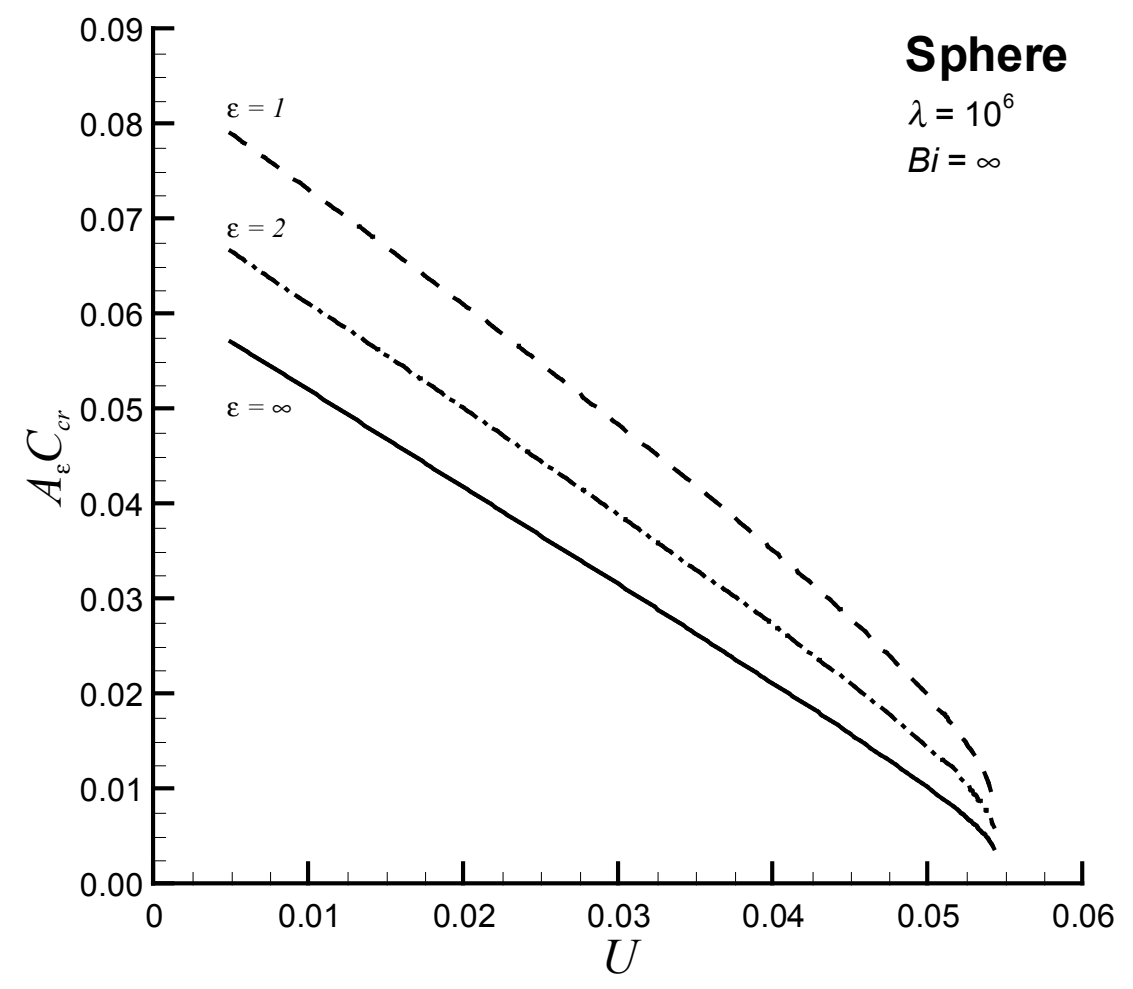

Figure 32. $A_{\varepsilon} C_{c r}$ versus $U$ curves for nonuniform spherical assemblies $\left(\lambda=10^{6}\right)$.

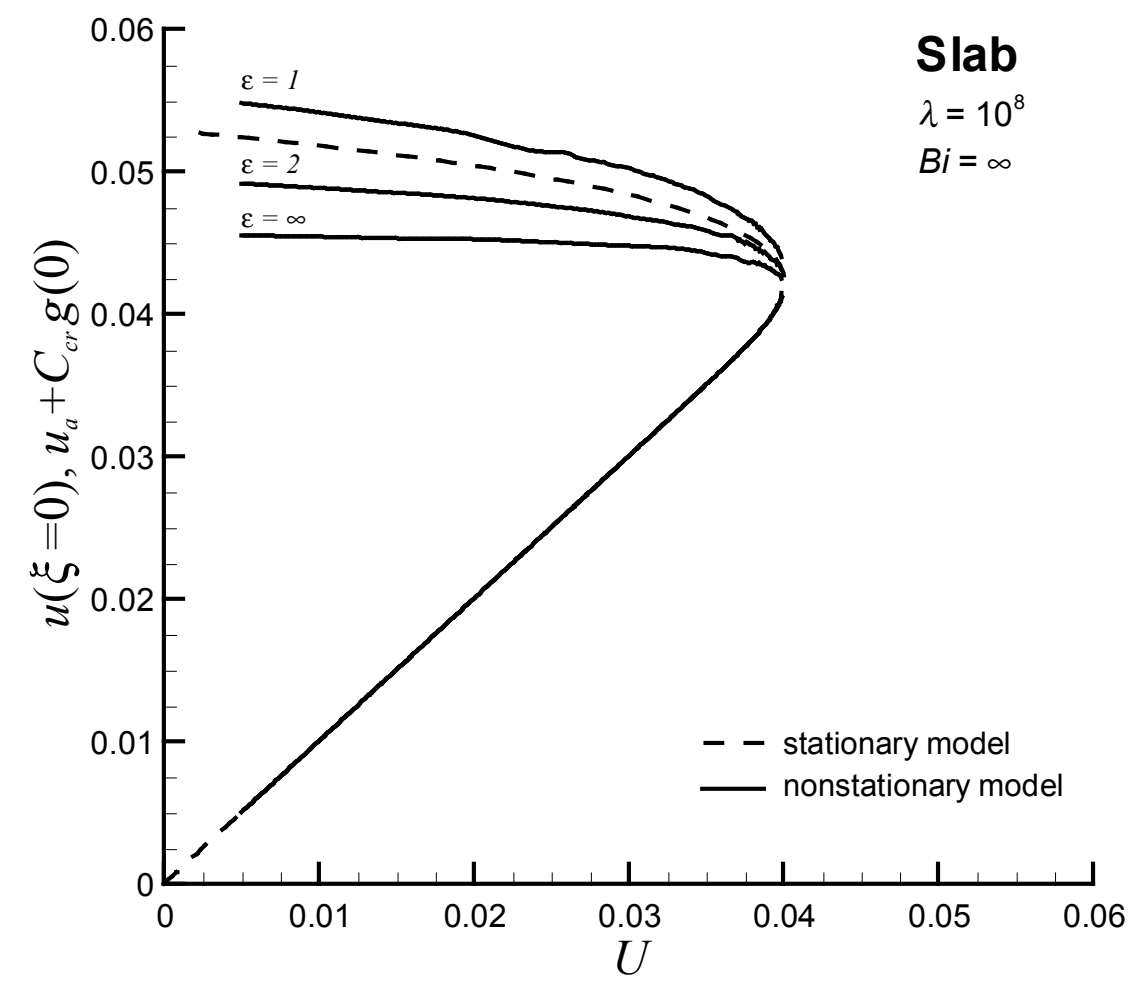

Figure 33. Criticality characteristics of nonuniform planar slab assemblies $\left(\lambda=10^{8}\right)$. 


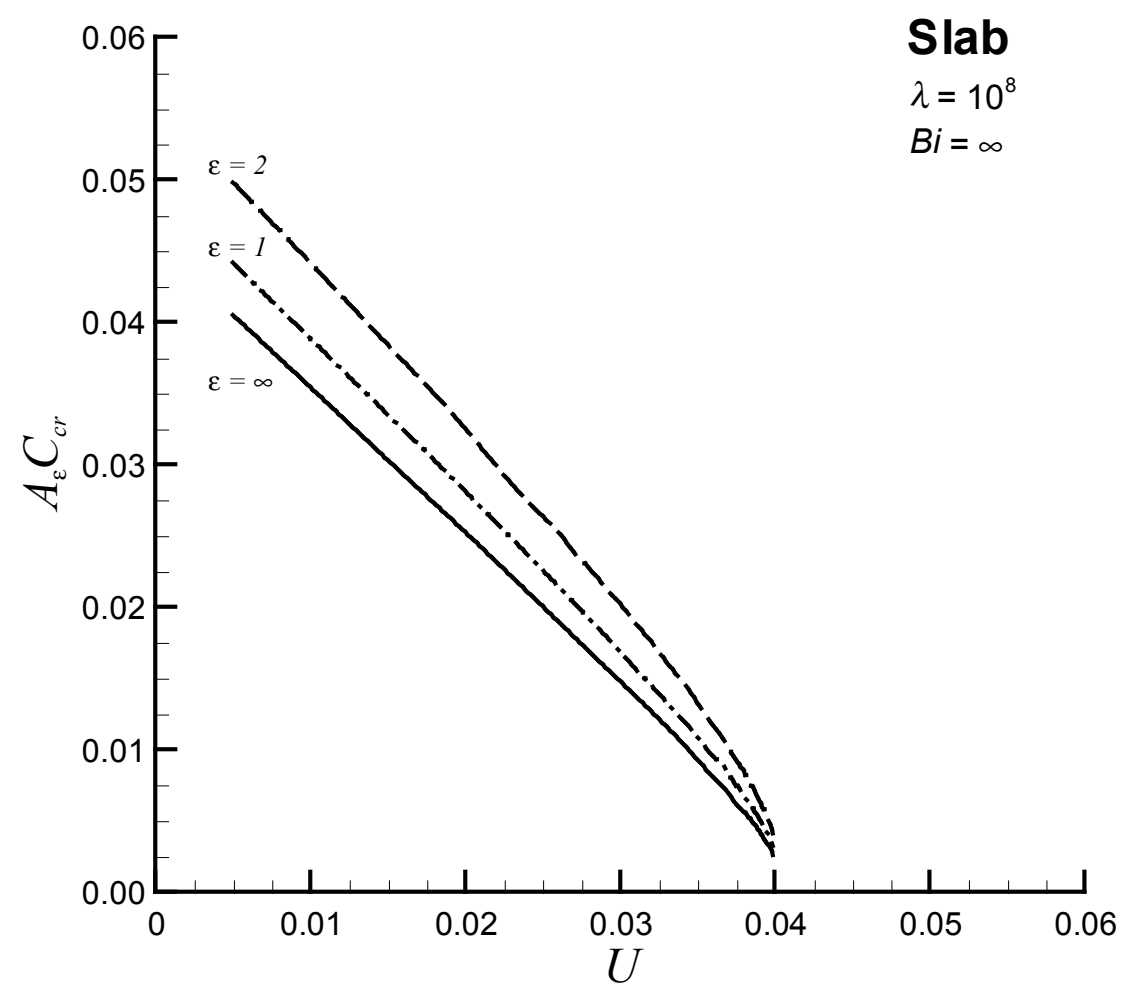

Figure 34. $A_{\varepsilon} C_{c r}$ versus $U$ curves for nonuniform planar slab assemblies $\left(\lambda=10^{8}\right)$.

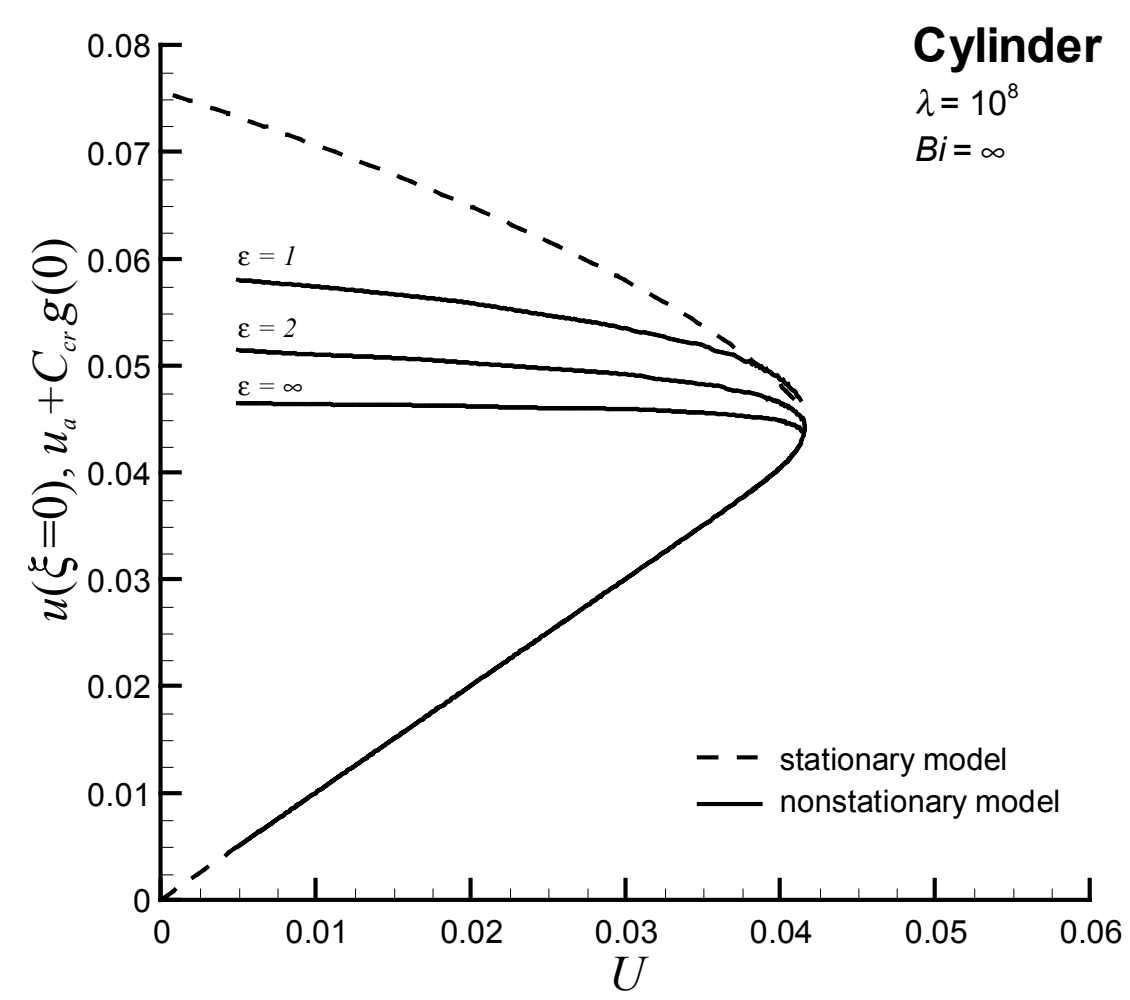

Figure 35. Criticality characteristics of nonuniform cylindrical assemblies $\left(\lambda=10^{8}\right)$. 


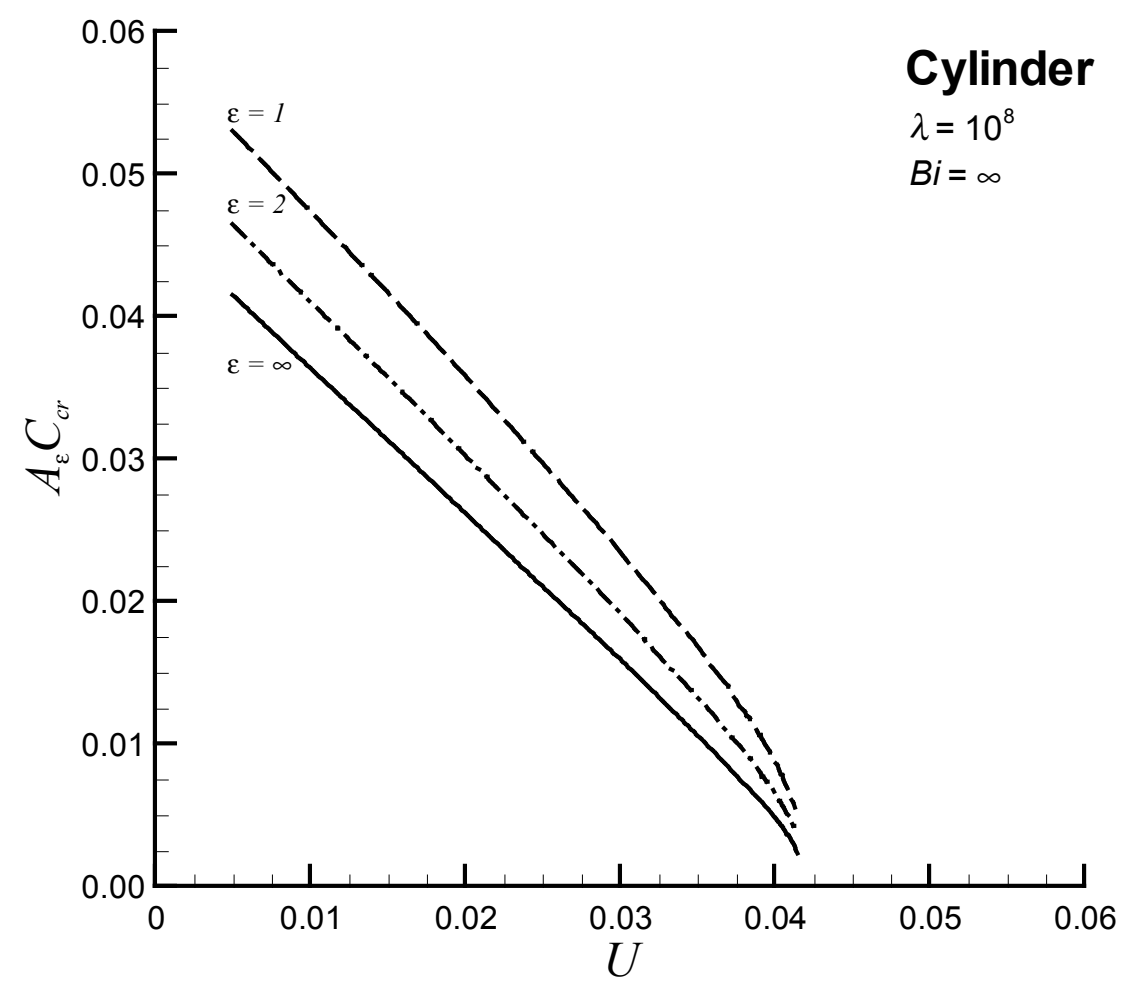

Figure 36. $A_{\varepsilon} C_{c r}$ versus $U$ curves for nonuniform cylindrical assemblies $\left(\lambda=10^{8}\right)$.

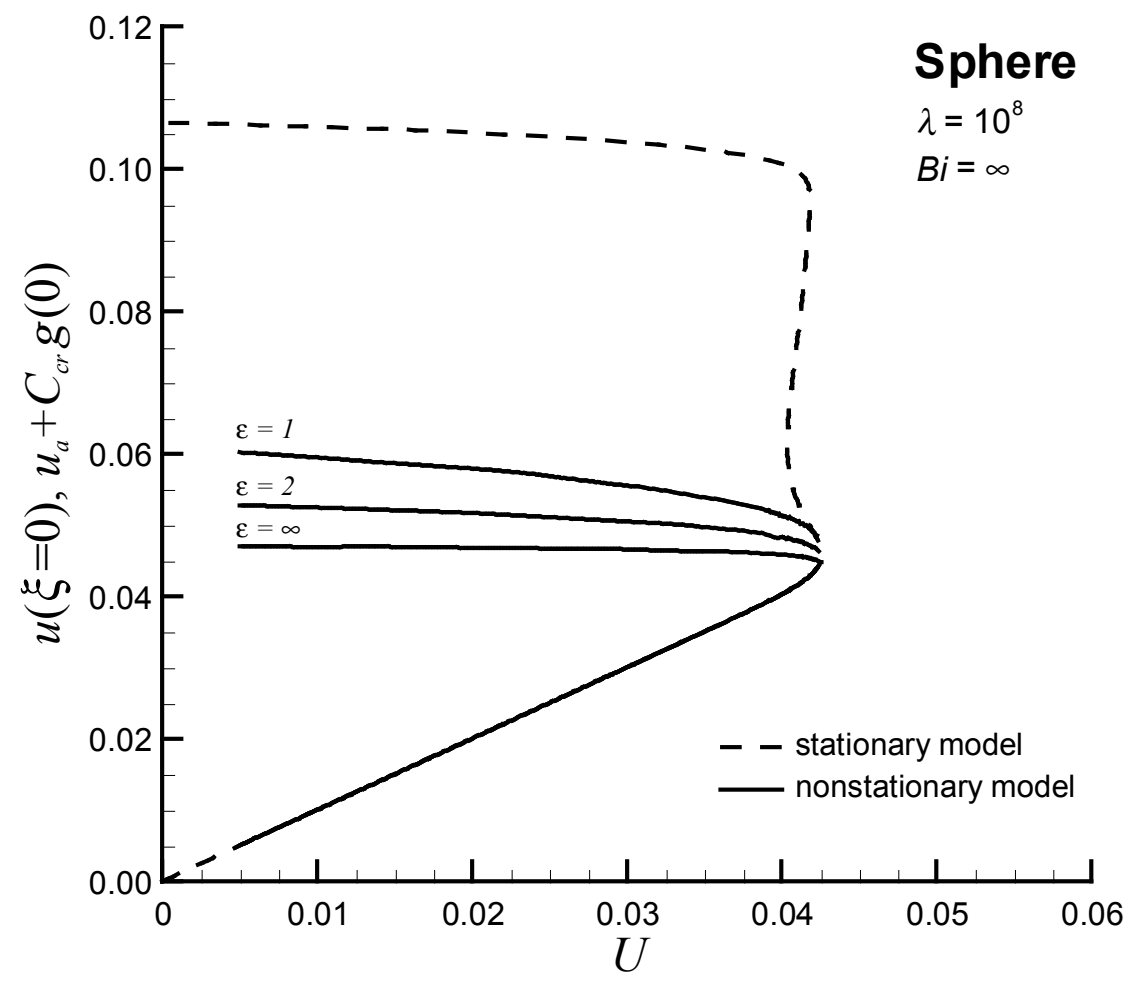

Figure 37. Criticality characteristics of nonuniform spherical assemblies $\left(\lambda=10^{8}\right)$. 


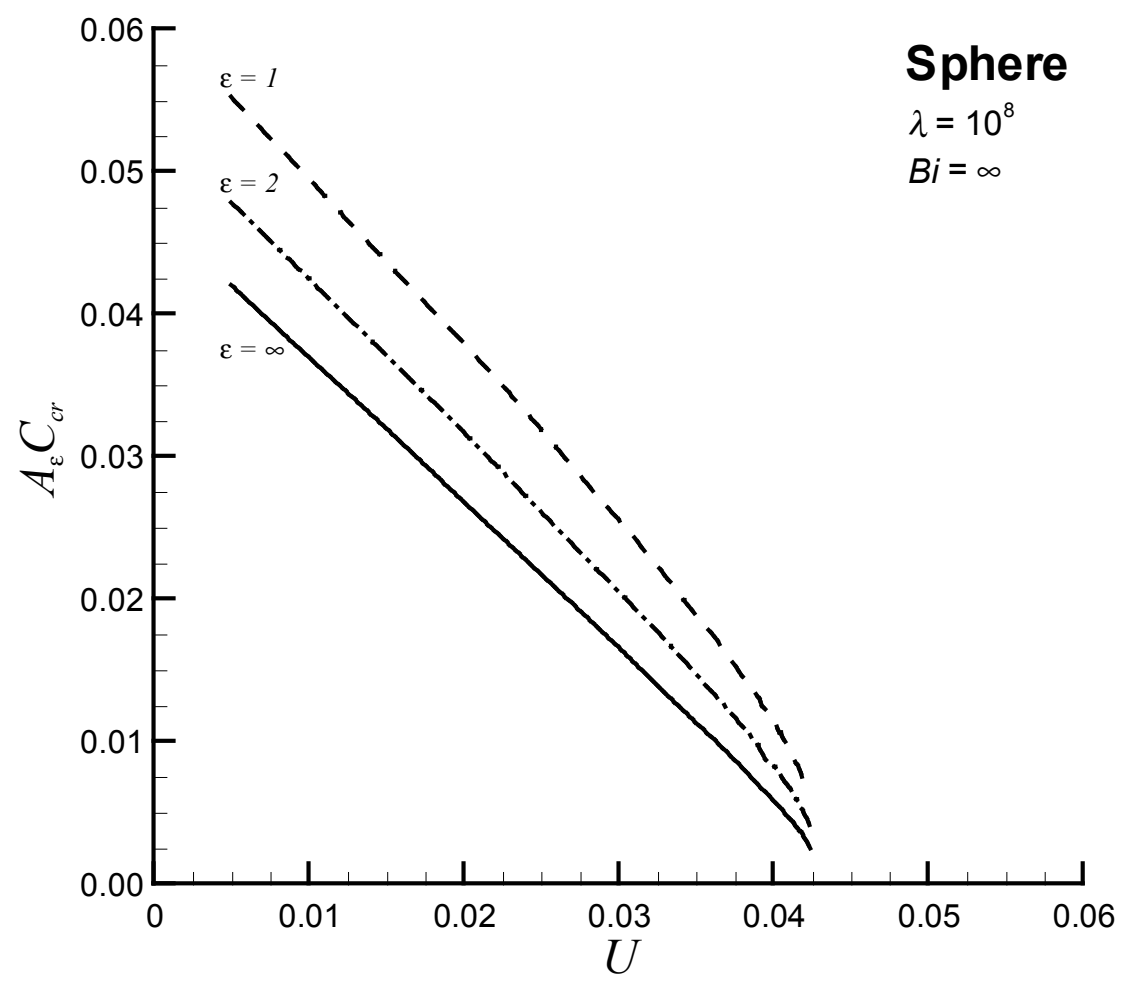

Figure 38. $A_{\varepsilon} C_{c r}$ versus $U$ curves for nonuniform spherical assemblies $\left(\lambda=10^{8}\right)$. 



\section{CORRELATION AND REDUCTION OF CRITICAL ASSEMBLY CONDITIONS}

In the previous section, an extensive numerical parametric study was undertaken to identify the critical threshold separating those initial conditions which lead to ignition/explosion and those which ultimately decay to a self-extinguishing quench state. Specifically, this study examined the influence of nonuniform thermal assemblies within the principal centrosymmetric solids. The outcome, a broad set of numerically constructed ignition thresholds over a wide range of values for the dimensionless eigenvalue, provided a reliable database for precisely determining ignition hazards given very specific physical conditions. From a deeper theoretical perspective, however, there is a natural desire to seek further means of reducing, ordering, and correlating these results such that the underlying mathematical structure may be revealed and expressed in the most compact and economical form possible. In this section, we pursue various methods of attack for achieving this objective.

First, we demonstrate that the critical threshold curves from the parametric study can be accurately correlated using a general second degree conic section. We then introduce a new dimensionless parameter into the resulting hyperbolic correlation and develop a simple relationship that collapses the entire data set from the parametric study onto a single universal line. This is an entirely new result which packs accurate predictive power within a highly compact form. Second, we formulate a conjecture and examine spatial moments of the initial energy content integral as an alternative means of reducing and correlating the critical threshold conditions.

\subsection{Correlating Forms and Structural Compaction}

At this stage, we are confronted with a large parametric database for which there are no obvious means of ordering and reducing the results in a straightforward deductive way. What we are seeking, of course, is a means of discovering and revealing hidden mathematical structure through an indirect process of intuitive reasoning. Under these circumstances, it is natural to examine the data in search of basic mathematical forms that can organize the results and provide improved insight. Of the numerous correlating forms that could be considered, conic sections offer one of the simplest and most widely useful constructs available. Here, we examine in detail the application of a second degree conic section correlation to the critical initial condition thresholds, and demonstrate effective compaction of the solution space's mathematical structure.

\subsubsection{Hyperbolic Correlation}

Close examination of the threshold curves in the $\left\{A_{\varepsilon} C_{c r}, U\right\}$ plane reveals structural features that tend to be associated with a hyperbolic conic section. To pursue the mathematical implications of this observation, we begin by recalling the general form of the origin centered hyperbola from elementary analytic geometry, as illustrated in figure 39. In this case, a point $(x, y)$ is on the hyperbola with vertices 


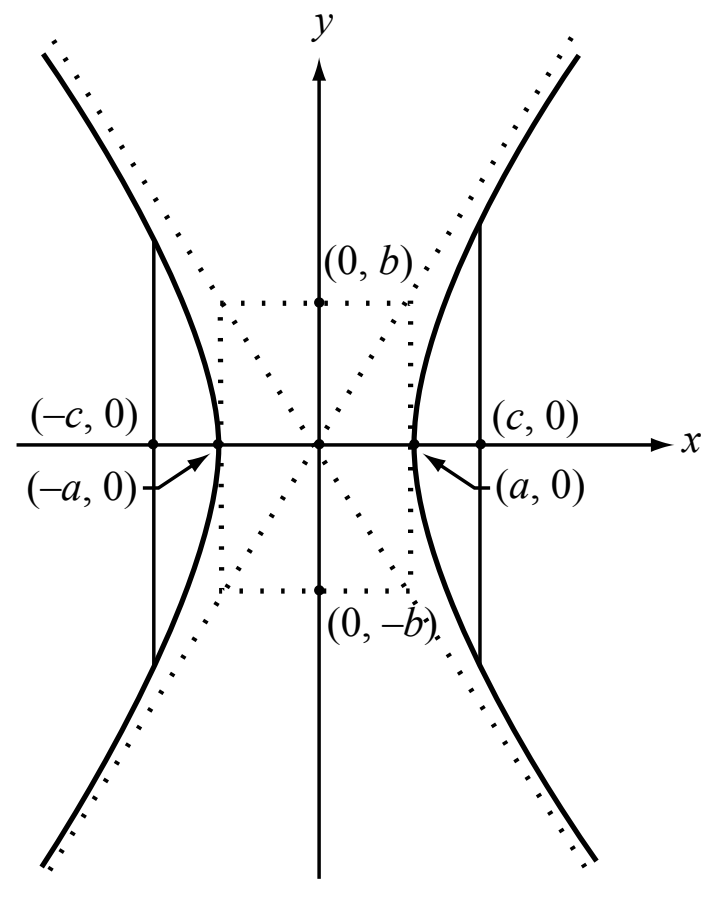

Figure 39. Origin-centered hyperbola.

$$
\frac{x^{2}}{a^{2}}-\frac{y^{2}}{b^{2}}=1
$$

$( \pm a, 0)$ and foci $( \pm c, 0)$ if, and only if, it satisfies the equation where $b^{2}=c^{2}-a^{2}$. Recall also that a hyperbola has a pair of slant asymptotes with slope $\pm b / a$, a distinct property that is in close correspondence to structural characteristics exhibited by the critical threshold curves.

To develop the desired hyperbolic correlation for the threshold curves in the $\left\{A_{\varepsilon} C_{c r}, U\right\}$ plane, it is now necessary to translate the coordinate axes according to a well known theorem which states that a point $(x, y)$ is on the hyperbola with center $(h, k)$, vertices $(h \pm a, k)$, and foci $(h \pm c, k)$ if and only if it satisfies the equation

$$
\frac{(x-h)^{2}}{a^{2}}-\frac{(y-k)^{2}}{b^{2}}=1 .
$$

where $b^{2}=c^{2}-a^{2}$. In this particular case, we identify the transverse and conjugate coordinates in the $\left\{A_{\varepsilon} C_{c r}, U\right\}$ plane as $x=U$ and $y=A_{\varepsilon} C_{c r}$ and define a new parameter $\Gamma=A_{\varepsilon} C_{c r}$ to arrive at the relation

$$
\frac{(U-h)^{2}}{a^{2}}-\frac{(\Gamma-k)^{2}}{b^{2}}=1 .
$$


At this point, we impose the obvious constraints $h=U_{c r}+a$ and $k=0$ and deduce our essential correlation equation

$$
\frac{\left(U-U_{c r}-a\right)^{2}}{a^{2}}-\frac{\Gamma^{2}}{b^{2}}=1 .
$$

Utilization of this correlation equation requires the introduction of certain additional assumptions in order to fully define values for the vertices and the foci. As a basis for the first assumption, we note that the slope of the critical threshold curve $\Gamma=A_{\varepsilon} C_{c r}$ away from the critical ambient temperature, $U_{c r}$, is approximately unity. Since this slope should match the slope of the slant asymptotes for the hyperbola, which by definition must take the value $\pm b / a$, we immediately deduce the constraint $b \approx-a$. In this case, equation (86) takes the simpler form

$$
\Gamma^{2}=\left(U-U_{c r}-a\right)^{2}-a^{2}
$$

The final remaining issue concerns definition of the vertex, $a$. From casual inspection of the critical threshold curves, we clearly anticipate that the value of $a$ must decrease as $\varepsilon$ increases. As such, we introduce the following two-parameter correlating expression:

$$
a=\alpha+\beta\left(\frac{2}{\varepsilon}\right),
$$

where $\alpha$ and $\beta$ are fitting parameters and $a$ is assumed to vary inversely with $\varepsilon$. Note that a multiplicative factor of 2 was introduced into equation (88) as a means of scaling $\beta$ such that $a=\alpha+\beta$ when $\varepsilon=2$.

The objective now is to deduce the best parameter values for fitting equation (87) to the precise critical threshold curves in the $\{\Gamma, U\}$ plane based on the complete data set from the parametric study of section 4 . The results of this fitting exercise yielded an array of optimal fitting parameters having a oneto-one correspondence with the array of $(n, \lambda)$ values from the parametric study. These fitting parameters are summarized in table 5. The actual hyperbolic correlation curves are shown with the precise computational threshold curves in figures 40-48 for each considered value of $\varepsilon$. Figures 40-42 depict results for the slab geometry $(n=0)$ for $\lambda=10^{4}, 10^{6}, 10^{8}$, respectively. Figures $43-45$ depict results for the cylindrical geometry $(n=1)$ for $\lambda=10^{4}, 10^{6}, 10^{8}$, respectively. Figures 46-48 depict results for the spherical geometry $(n=2)$ for $\lambda=10^{4}, 10^{6}, 10^{8}$, respectively.

Inspection of these figures clearly demonstrates that the hyperbolic correlation provides an excellent fit to the precise computational results over the full range of $\varepsilon$ and $\lambda$ values under consideration. Thus, we conclude that the mathematical structure of the complete solution space is accurately captured by a simple hyperbolic conic section, as defined by equation (87), when using a two-parameter correlating expression for the vertex, as given by equation (88). Variations in the fitting parameters appear to be smooth and well behaved, and it is clear that $\alpha$ and $\beta$ could further be expressed as analytical functions of $n$ and $\lambda$, as well. Here, however, we shall be content to leave them in tabulated form, only. 
Table 5. Optimal fitting parameters for hyperbolic correlation.

\begin{tabular}{|c|c|c|c|}
\hline Slab $(\boldsymbol{n}=\mathbf{0})$ & $\boldsymbol{\lambda}=\mathbf{1 0}^{\mathbf{4}}$ & $\boldsymbol{\lambda}=\mathbf{1 0}^{\mathbf{6}}$ & $\boldsymbol{\lambda}=\mathbf{1 0}^{\mathbf{8}}$ \\
\hline$\alpha$ & 0.0200 & 0.0100 & 0.0060 \\
$\beta$ & 0.0170 & 0.0080 & 0.0052 \\
\hline \hline Cylinder $(\boldsymbol{n}=\mathbf{1})$ & $\lambda=\mathbf{1 0}^{\mathbf{4}}$ & $\boldsymbol{\lambda}=\mathbf{1 0}^{\mathbf{6}}$ & $\boldsymbol{\lambda}=\mathbf{1 0}^{\mathbf{8}}$ \\
\hline$\alpha$ & 0.0200 & 0.0097 & 0.0056 \\
$\beta$ & 0.0250 & 0.0110 & 0.0065 \\
\hline Sphere $(\boldsymbol{n}=\mathbf{2})$ & $\lambda=\mathbf{1 0}^{\mathbf{4}}$ & $\boldsymbol{\lambda}=\mathbf{1 0}^{\mathbf{6}}$ & $\lambda=\mathbf{1 0} \mathbf{8}$ \\
\hline$\alpha$ & 0.0200 & 0.0090 & 0.0050 \\
$\beta$ & 0.0300 & 0.0130 & 0.0075 \\
\hline
\end{tabular}

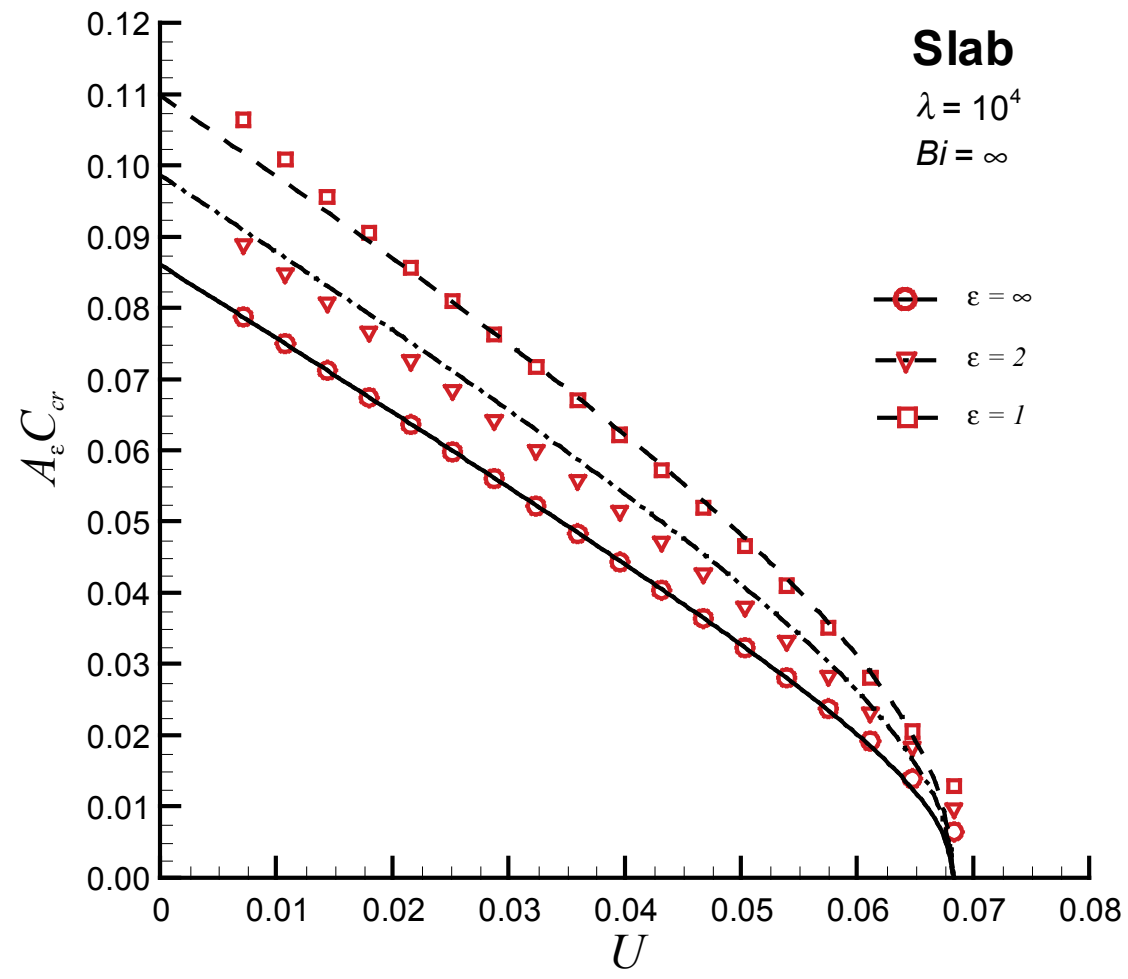

Figure 40. $A_{\varepsilon} C_{c r}$ versus $U$ results for nonuniform planar slab assemblies $\left(\lambda=10^{4}\right)$. Precise computational value - symbols. Hyperbolic correlation-curves. 


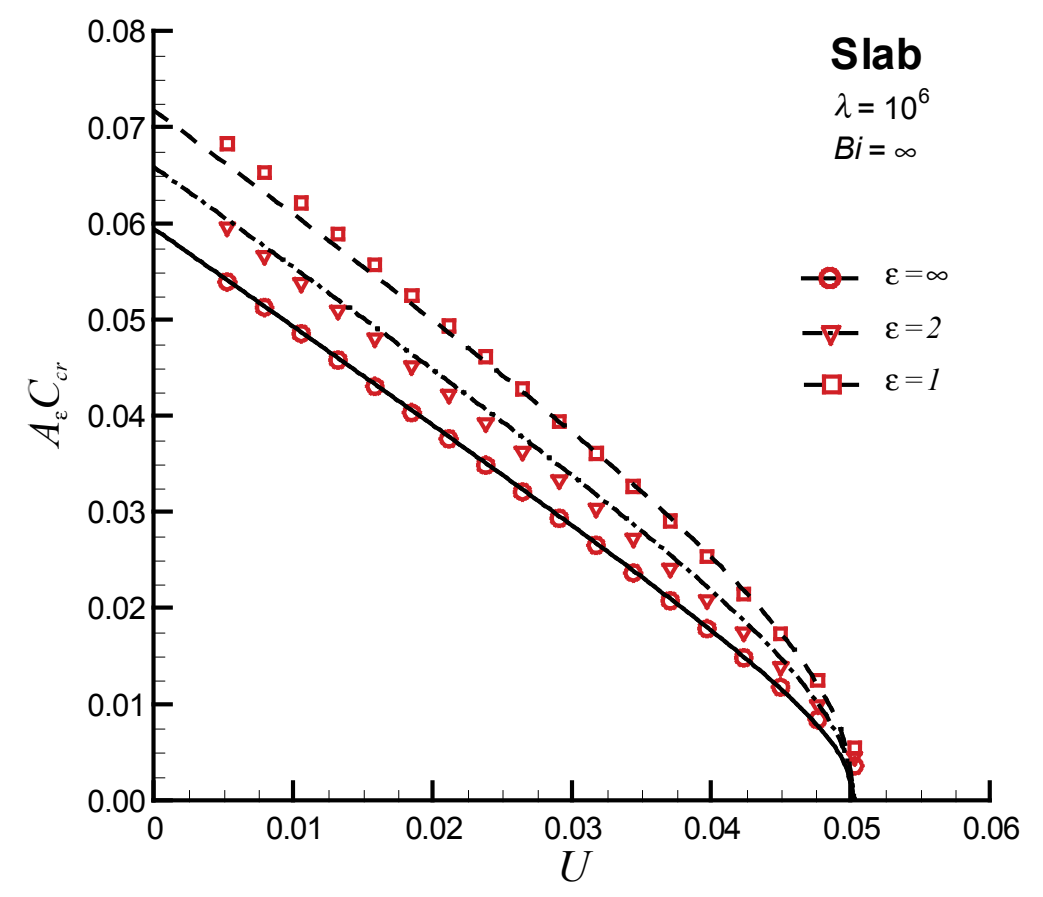

Figure 41. $A_{\varepsilon} C_{c r}$ versus $U$ results for nonuniform planar slab assemblies $\left(\lambda=10^{6}\right)$. Precise computational value - symbols. Hyperbolic correlation-curves.

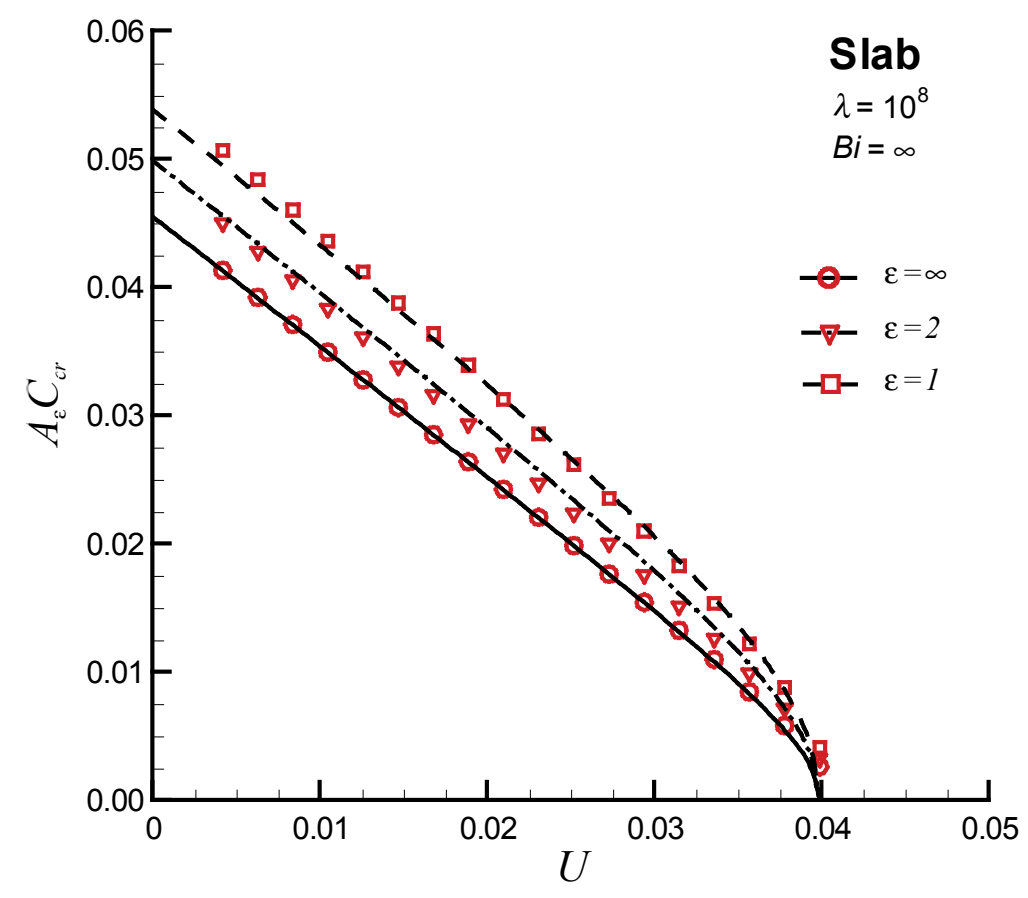

Figure 42. $A_{\varepsilon} C_{c r}$ versus $U$ results for nonuniform planar slab assemblies $\left(\lambda=10^{8}\right)$. Precise computational value - symbols. Hyperbolic correlation-curves. 


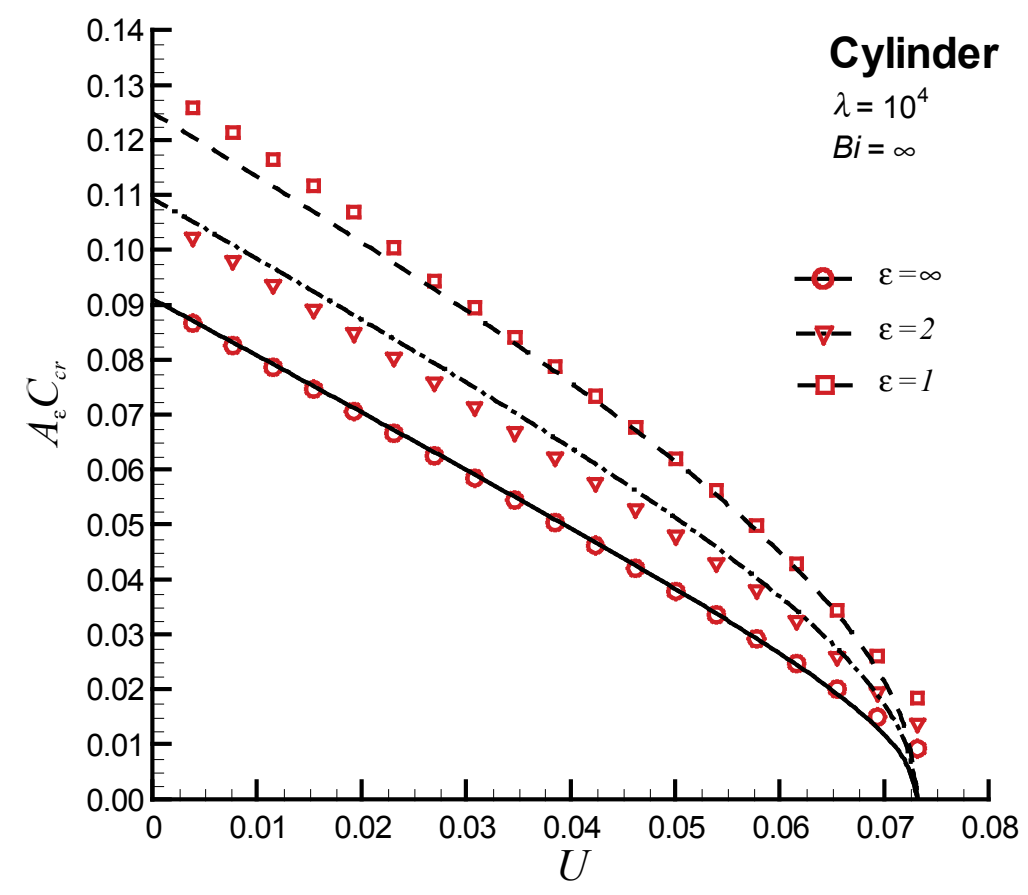

Figure 43. $A_{\varepsilon} C_{c r}$ versus $U$ results for nonuniform cylindrical assemblies $\left(\lambda=10^{4}\right)$. Precise computational value - symbols. Hyperbolic correlation-curves.

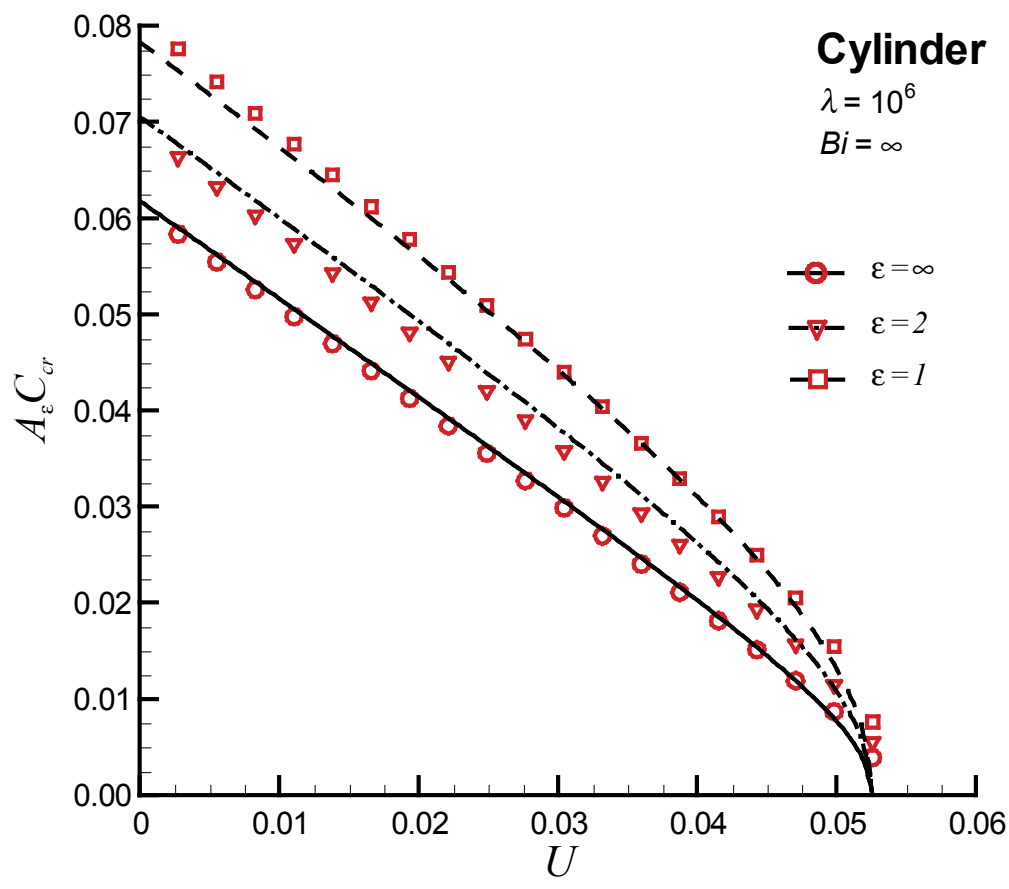

Figure 44. $A_{\varepsilon} C_{c r}$ versus $U$ results for nonuniform cylindrical assemblies $\left(\lambda=10^{6}\right)$. Precise computational value - symbols. Hyperbolic correlation-curves. 


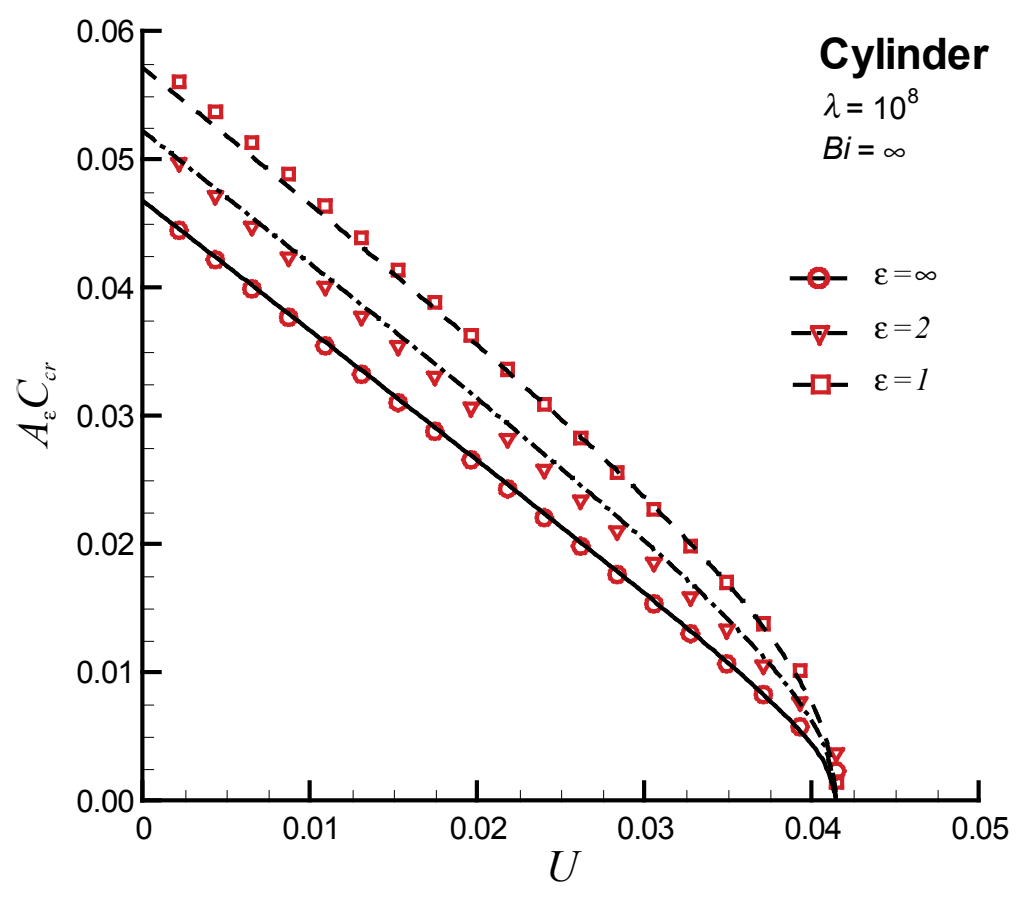

Figure 45. $A_{\varepsilon} C_{c r}$ versus $U$ results for nonuniform cylindrical assemblies $\left(\lambda=10^{8}\right)$. Precise computational value - symbols. Hyperbolic correlation-curves.

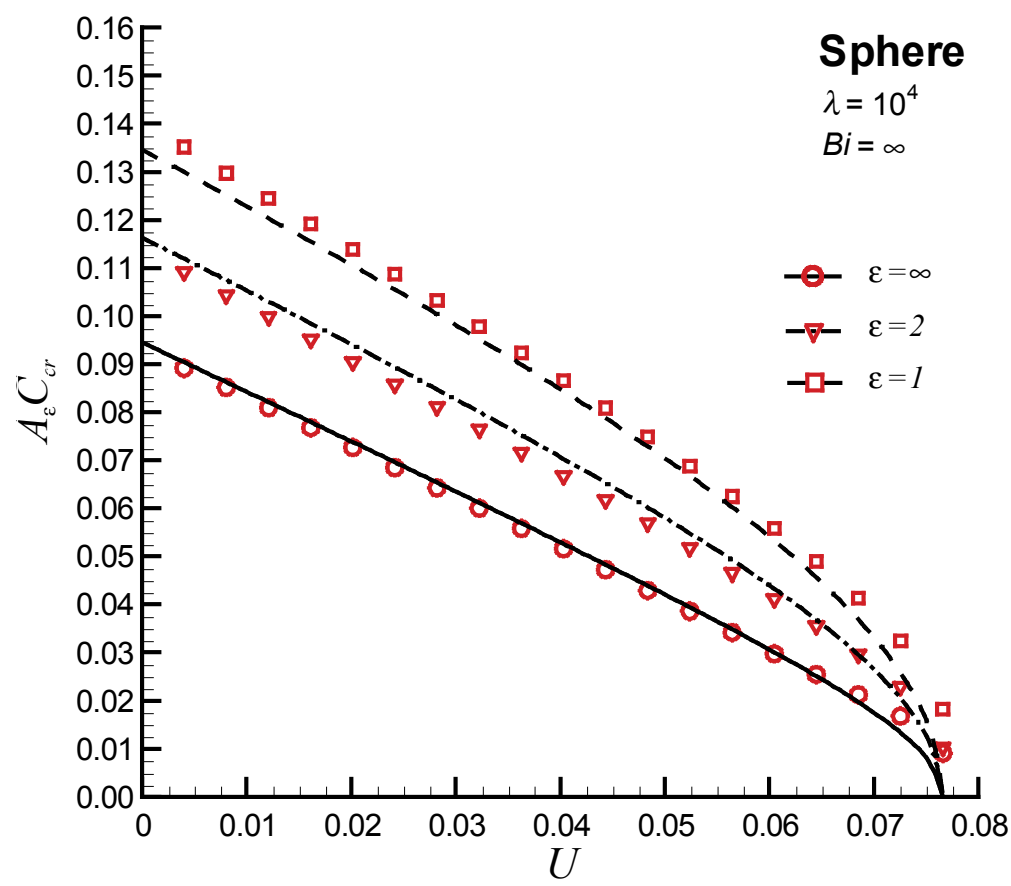

Figure 46. $A_{\varepsilon} C_{c r}$ versus $U$ results for nonuniform spherical assemblies $\left(\lambda=10^{4}\right)$. Precise computational value-symbols. Hyperbolic correlation-curves. 


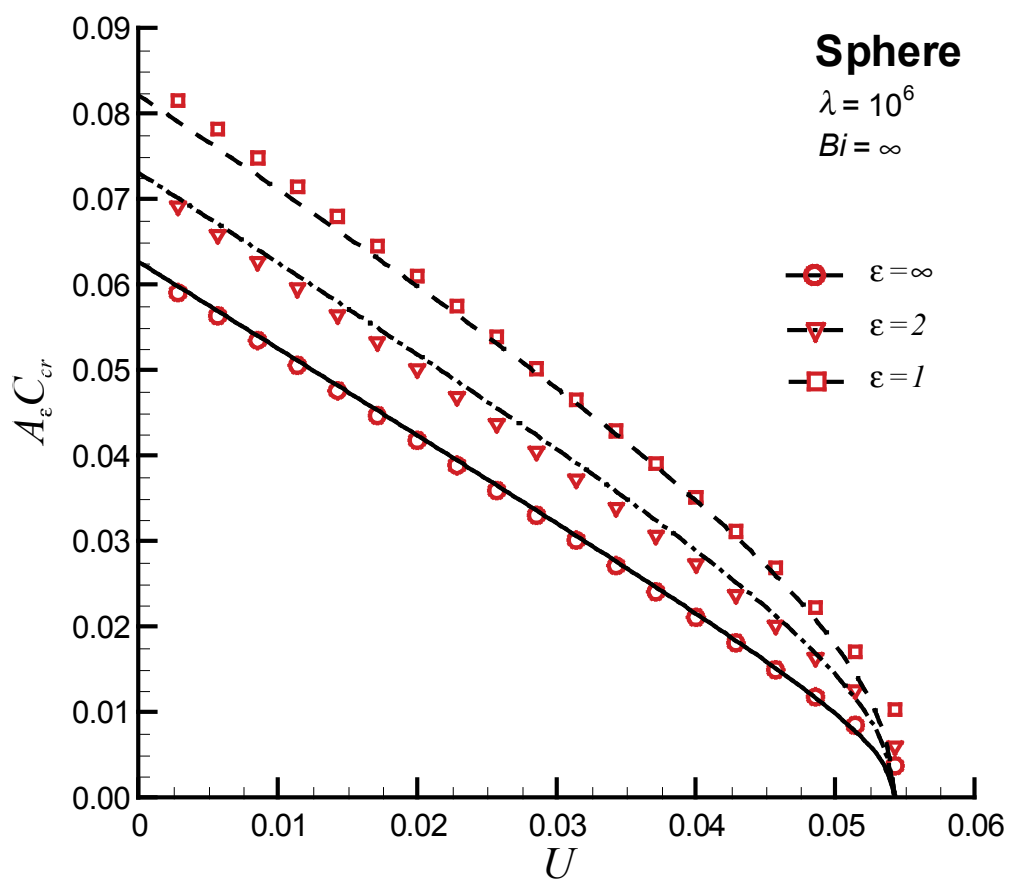

Figure 47. $A_{\varepsilon} C_{c r}$ versus $U$ results for nonuniform spherical assemblies $\left(\lambda=10^{6}\right)$.

Precise computational value — symbols. Hyperbolic correlation- curves.

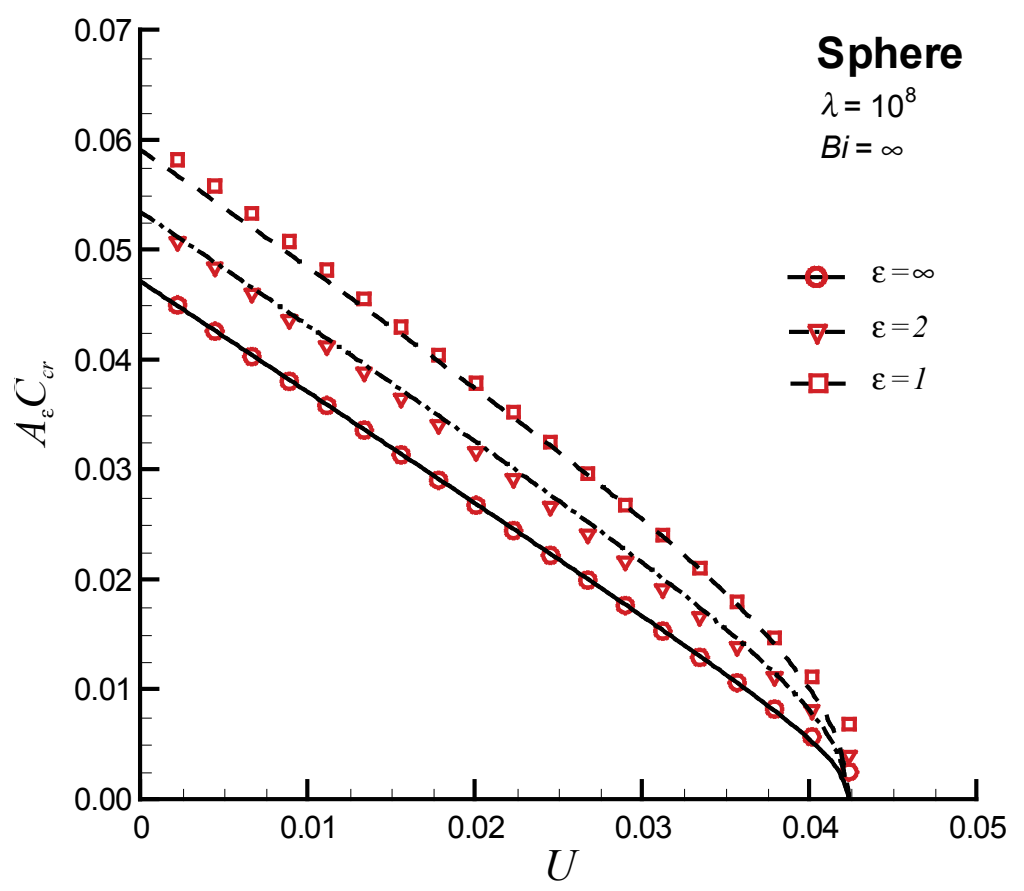

Figure 48. $A_{\varepsilon} C_{c r}$ versus $U$ results for nonuniform spherical assemblies $\left(\lambda=10^{8}\right)$. Precise computational value-symbols. Hyperbolic correlation-curves. 


\subsubsection{Reduction to Compact Universal Form}

Application of the hyperbolic correlation to the complete parametric solution space provided pleasing enough results to encourage further examination of the mathematical implications. For example, an immediate follow-on question arises as to whether it is possible to achieve additional reduction through the deduction of a compact universal correlating form. To pursue this question in detail, we revisit our hyperbolic correlation in the simplified form of equation (87).

Our first course of action is to define a new dimensionless temperature $\Delta U=U_{c r}-U$ so that equation (87) may be written as

$$
\Gamma^{2}=(-\Delta U-a)^{2}-a^{2}
$$

Then, we expand the bracketed square in order to find

$$
\Gamma^{2}=\Delta U^{2}+2 a \Delta U
$$

or

$$
\Delta U^{2}+2 a \Delta U-\Gamma^{2}=0
$$

Noting that this equation is quadratic in $\Delta U$, we may immediately write an expression defining $\Delta U$ explicitly in terms of $\Gamma$ :

$$
\Delta U=\sqrt{\Gamma^{2}+a^{2}}-a
$$

We now have a convenient explicit correlating form that completely captures the essential mathematical structure of the parametric solution space, and it is strikingly simple. An even simpler form can now be had by defining the right hand side of equation (92) as a new dimensionless criticality parameter, $\hat{\Gamma}=\sqrt{\Gamma^{2}+a^{2}}-a$. We therefore arrive at the universal linear correlating form

$$
\hat{\Gamma}=\Delta U
$$

This result is utterly simple and, in a sense, mathematically beautiful since it collapses the entire solution space onto a single line in the $\{\hat{\Gamma}, \Delta U\}$ plane. The results of this exercise are summarized in figures 49 , 50, and 51 for the slab, cylinder, and sphere, respectively. Although all of the data collapses to the same universal line, the results are depicted separately by geometry in order to avoid excess clutter.

The fit to the universal line is generally good except for some slight deviation in the low ambient temperature region where $U<<U_{c r}$. This deviation arises from the fact that the actual threshold curve begins to inflect as it nears and passes through the $U=0$ axis, whereas the hyperbolic correlation requires a continuous merging with the slant asymptote. Even there, the deviation is not extremely large. In general, the fit is better at large $\varepsilon$ and slightly degrades as the value becomes smaller and the initial thermal distribution has a higher degree of nonuniformity. 


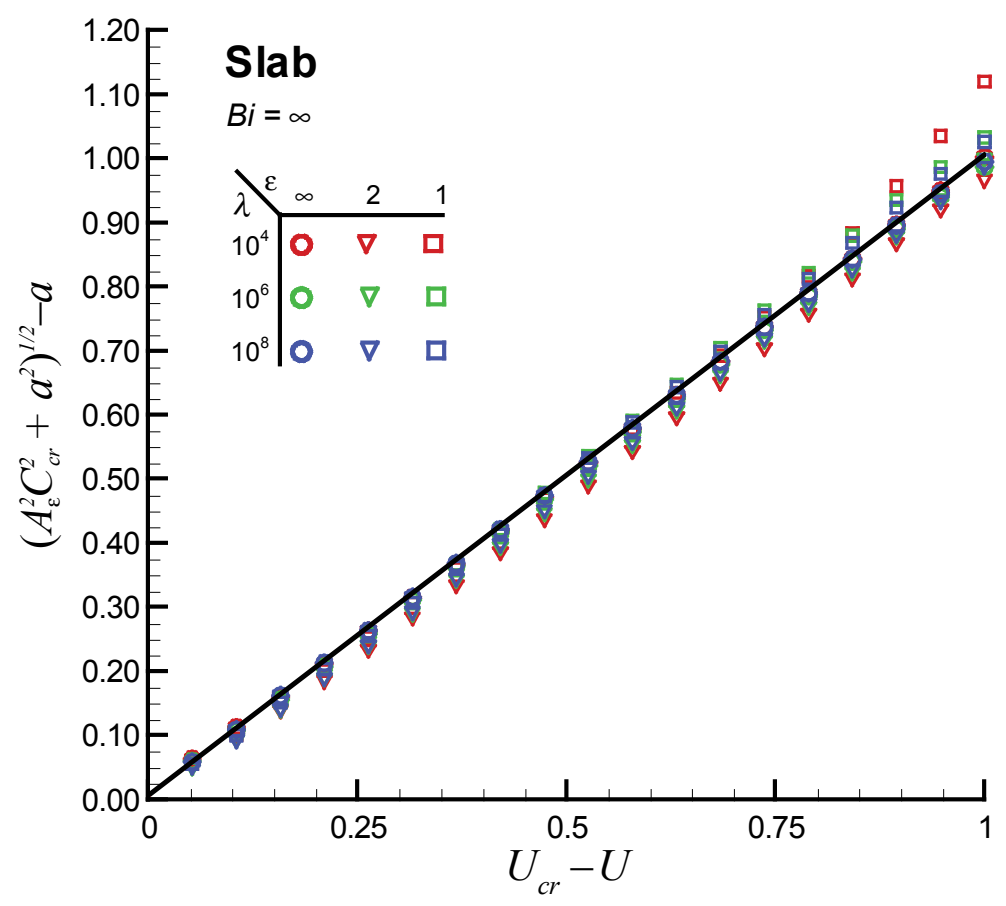

Figure 49. Universal correlating line. Slab $(n=0)$.

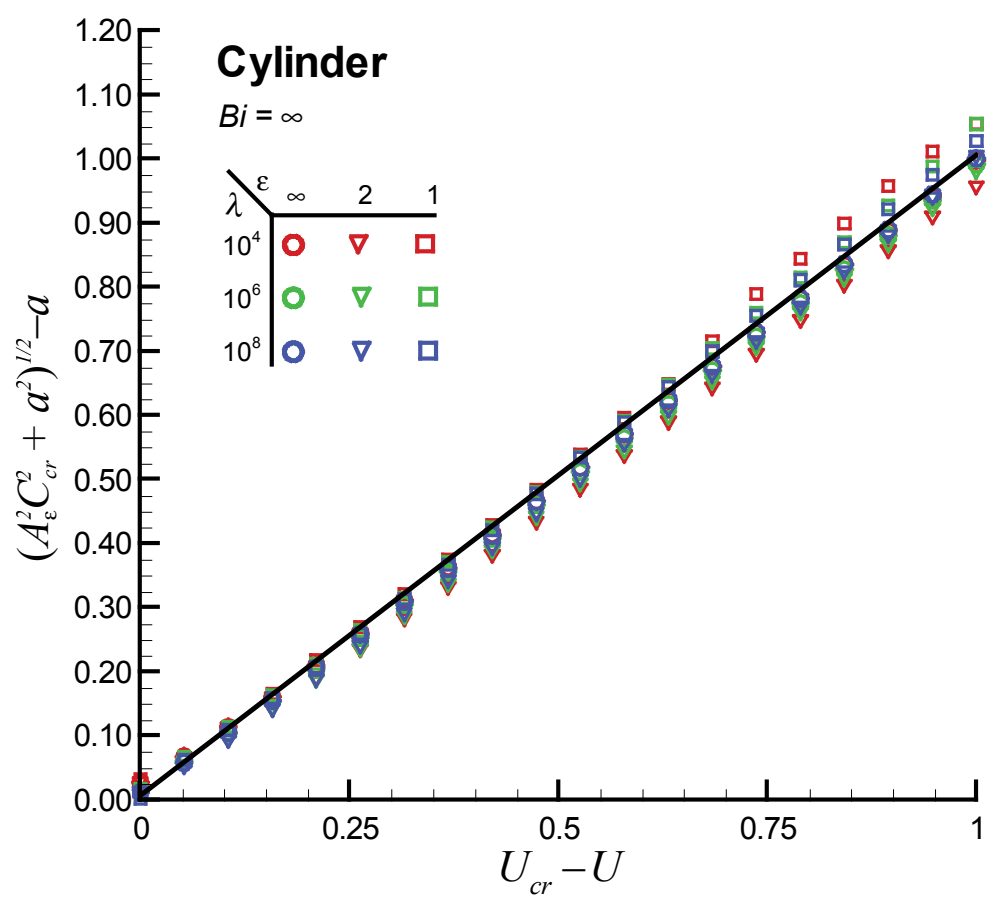

Figure 50. Universal correlating line. Cylinder $(n=1)$. 


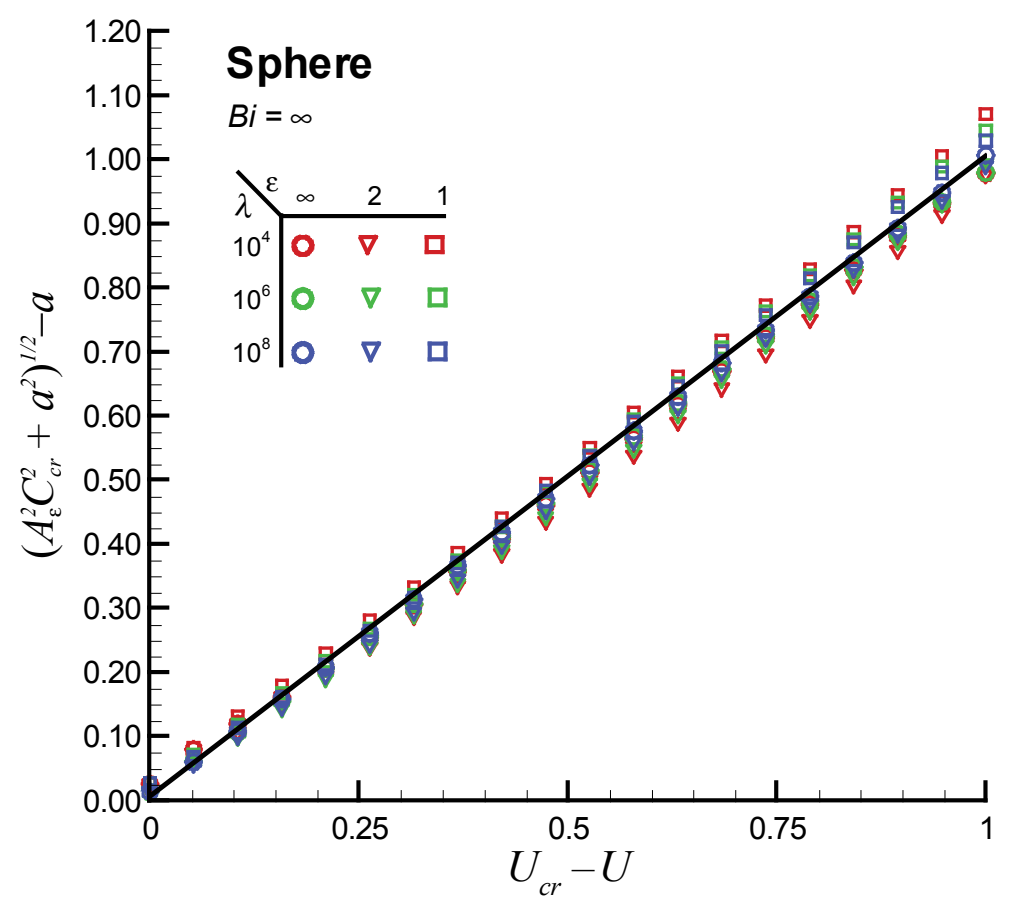

Figure 51. Universal correlating line. Spherical $(n=2)$.

\subsection{Spatial Moments of Energy Content Integral}

Intuitively, we expect that the ultimate fate of a combustible assembly should have a direct mathematical coupling to its initial energy distribution. We further anticipate that the predisposition for this assembly to either ignite or quench may be conveniently and compactly expressed as an integral over this initial energy distribution. Our purpose then is to rigorously define the initial energy content integral and precisely state a mathematical conjecture relating spatial moments of this integral to the fate of thermally nonuniform assemblies of combustible substances. The results of the previous computational parametric study may then used as a basis for thoroughly testing the conjecture and establishing a provisional basis for its acceptance and application to industrial fire problems of great practical interest.

\subsubsection{Definitions and Conjecture}

From physical reasoning, we assert that the critical condition for ignition of a thermally nonuniform combustible assembly depends largely upon the initial energy concentration or density, particularly within a centralized hot region as represented by the one-parameter shape profile of figure 20. Here, the hot spot has been centered on the axis of symmetry as a worse case representation since the dispersion and removal of excess heat is most impeded under these circumstances.

We therefore begin our development with a fundamental definition of energy density $e$ :

$$
e=\rho c_{p} T
$$


where the variables on the right hand side are all as previously defined. The total energy content $E$ must then follow from a volume integral over the full assembly:

$$
E=\oiiint e d V
$$

Now, recall our previous definitions for the nondimensional variables $\alpha=\rho c_{p} / k$ and $u=R T / E_{a}$ and eliminate $c_{p}$ and $T$ in equation (94) to obtain a dimensionless local energy density in the form:

$$
\hat{e}=e /\left(\frac{\alpha k E_{a}}{R}\right)=u .
$$

Pursuing a similar train of thought, we introduce the following definition for the dimensionless total energy content:

$$
\hat{E}=E /\left(\frac{\alpha k E_{a}}{R}\right)
$$

and reformulate the volume integral of equation (95) to obtain

$$
\hat{E}=\oiiint \hat{e} d V=\oiiint u d V
$$

Note that the total energy content within the assembly has been succinctly and conveniently expressed as the volume integral of the dimensionless reactant temperature over the complete assembly volume. With more specificity, we now apply the energy content integral to the principal centrosymmetric solids and reduce to the simplified expression

$$
\hat{E}=\int_{0}^{1} \hat{e}(\xi, t) d \xi=\int_{0}^{1} u(\xi, t) d \xi,
$$

which is to be evaluated at time $t=0$. As a point of clarification, it should be noted that certain geometrical multiplication factors have been neglected here in order to achieve commonality of form.

Let us proceed further by considering spatial moments of our dimensionless energy content integral. The conventional approach for defining a spatial moment is to weight the kernel of the integral by the distance from the origin (i.e., the moment arm) raised to some arbitrary power, which defines the order of the moment. Naturally, this places less weight on contributions to the integral that are closer to the origin than those that are farther away. Because our hot spot is located on the axis of symmetry, however, we wish to place more weight on integral contributions that are closest to the origin. Thus, we henceforth define our moment arm using the factor $(1-\xi)$ and define the $m$ th order spatial moment for the $n$th class geometry as

$$
\psi_{m, n}=\int_{0}^{1}(1-\xi)^{m} u(\xi, 0) d \xi
$$


Recalling the generalized one-parameter initial shape profile from section 4, we deduce the practical working form

$$
\psi_{m, n}=\int_{0}^{1}(1-\xi)^{m}\left[U+C_{c r} g(\xi)\right] d \xi=\int_{0}^{1}(1-\xi)^{m}\left[U+C_{c r} A_{\mathcal{E}}\left(1-\xi^{\mathcal{E}}\right)\right] d \xi
$$

It should be clear that this expression is explicitly dependent on the geometrical index $n$ and that the influence of geometry on the spatial moment is carried entirely within the critical constant, $C_{c r}$.

We now state, based principally on intuitive reasoning, the following mathematical conjecture:

Given any thermally nonuniform assembly in the form of a principal centrosymmetric solid, there exist nonstationary solutions to the Burnell-GrahamEagle-Gray-Wake reaction-diffusion equation, under the constraint $B i \rightarrow \infty$, for which certain order- $m$ spatial moments of the critical total energy content integral, $\psi_{n, m}$, are invariant with changes in the dimensionless ambient temperature, $U$, and the initial shape profile parameter, $\varepsilon$, within some quantifiable error band, and are therefore functionally dependent on the dimensionless eigenvalue, $\lambda$, only.

\subsubsection{Provisional Validation}

As a means of generating reliable quantitative evidence for the previously stated conjecture, we now consider the first and second order spatial moments $(m=1,2)$ of the critical total energy content integrals over the full parameter space of the computational study. Evaluation of these integrals was effected by numerically integrating equation (101) using a cubic spline interpolation procedure to define $C_{c r} A_{\varepsilon}$ between available data points. The results of these evaluations are summarized in figures 52-57, which display the variation in $\bar{\psi}_{m, n}$ with $U / U_{c r}$ over the entire parameter range of the study.

Inspection of the resulting first- and second-order spatial moments provides provisional confirmation of our conjecture and indicates that the second-order moment, which has a narrower error band, may serve as a more accurate basis of prediction. It should also be noted that the error band exhibits a tendency to narrow as the value of $\lambda$ increases. Results for the third order spatial moments, which were also computed but not displayed, showed a re-widening of the error band. Apparently, the second order spatial moment satisfies our conjecture with the least variance and the smallest realizable error band. Spatial moments of noninteger order were not considered.

If we accept these results as provisional validation for the conjecture, we now have at hand a simple method for rapidly calculating fire hazard risks for thermally nonuniform assemblies of selfheating materials. Assuming that the assembly temperature profile $u(\xi)$ is known with a reasonable degree of confidence and that the physical characteristics of the substance are well enough defined to compute $\lambda$ to good accuracy, one may simply evaluate the spatial moment from equation (100) and compare this result with the mean value of the moments determined by this study. If the computed spatial moment is near to or greater than the mean reference value, at the specified value of $\lambda$, then the risk for spontaneous ignition should be considered high. If the computed spatial moment is less than that mean reference value, one may safely assume that the risk is low or negligible, depending on the magnitude of the difference. Arithmetically averaged values for the spatial moments integrals $\bar{\psi}_{n, m}$ are summarized in table 6 for convenient use and are also plotted as a function of $\lambda$ in figure 58. 


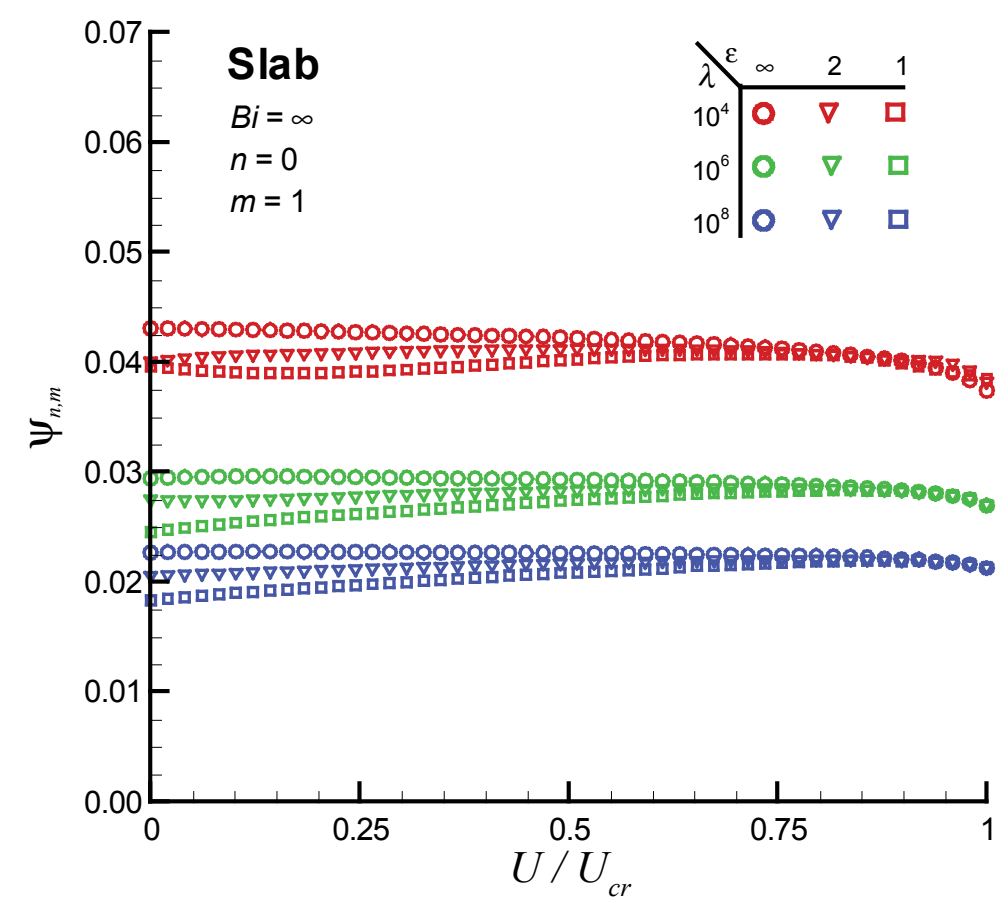

Figure 52. $\psi_{n, m}$ versus $U / U_{c r}$ for nonuniform slab assemblies $(n=0, m=1)$.

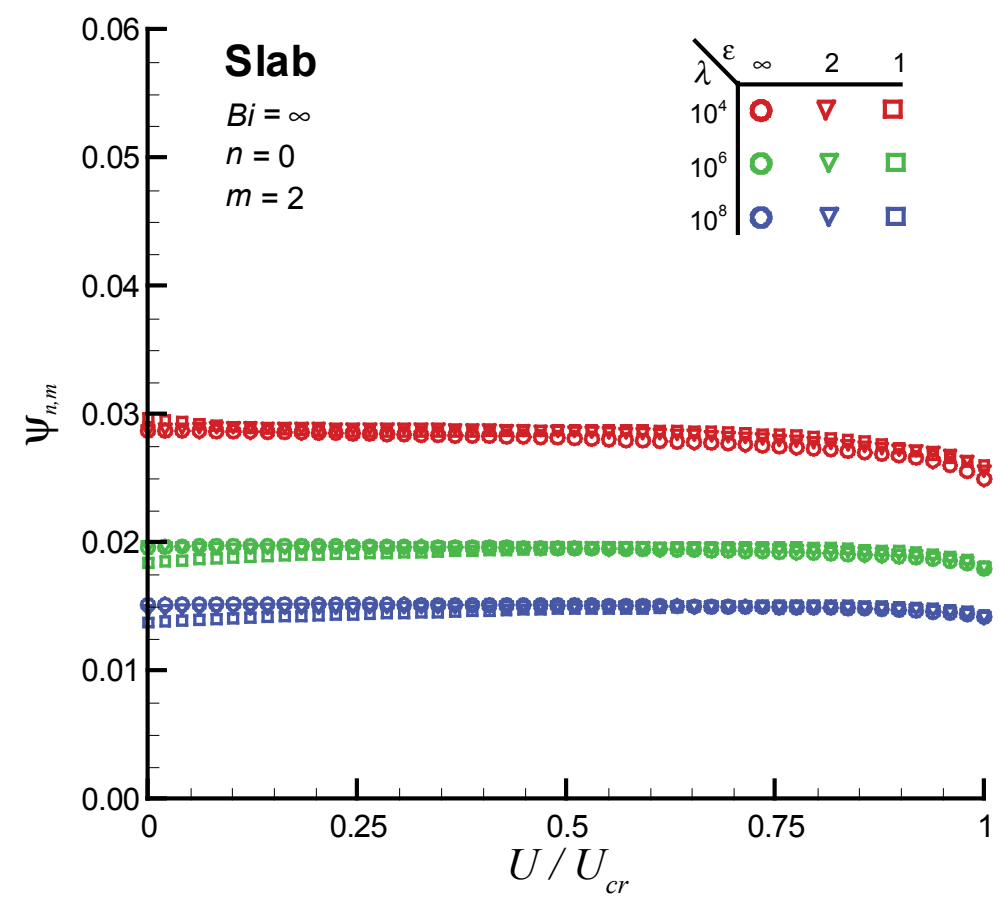

Figure 53. $\psi_{n, m}$ versus $U / U_{c r}$ for nonuniform slab assemblies $(n=0, m=2)$. 


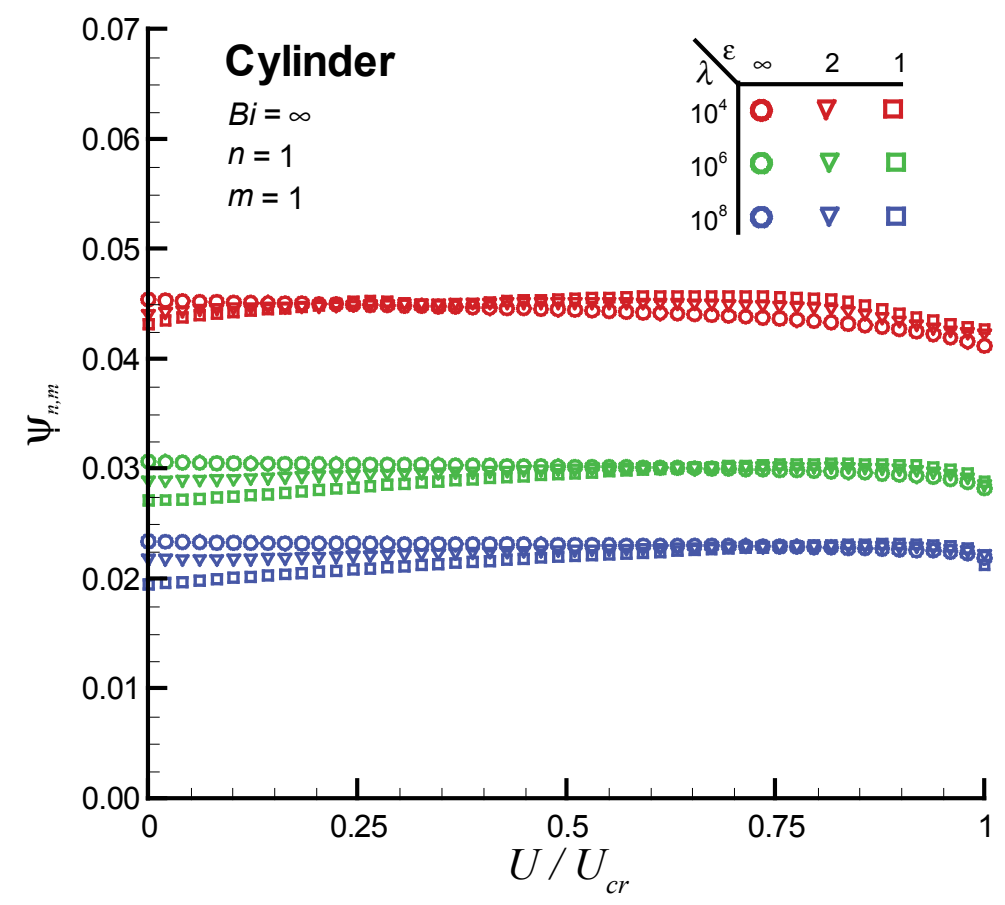

Figure 54. $\psi_{n, m}$ versus $U / U_{c r}$ for nonuniform cylindrical assemblies $(n=1, m=1)$.

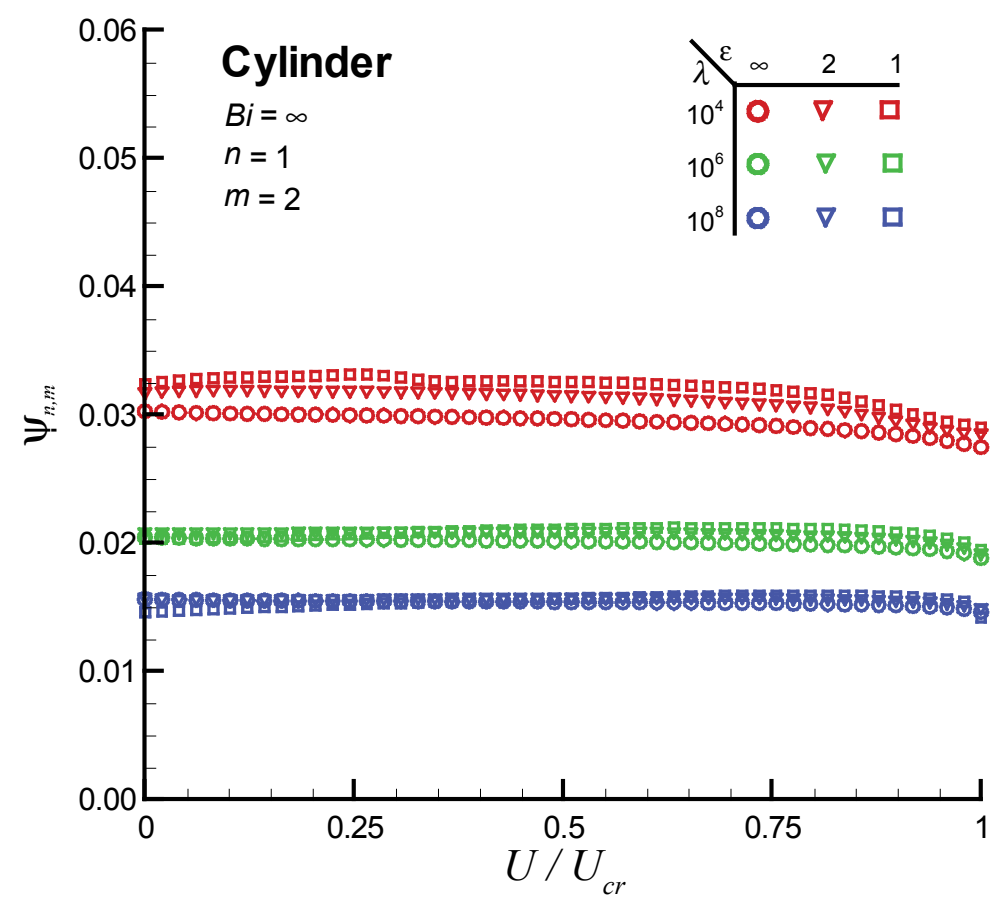

Figure 55. $\psi_{n, m}$ versus $U / U_{c r}$ for nonuniform cylindrical assemblies $(n=1, m=2)$. 


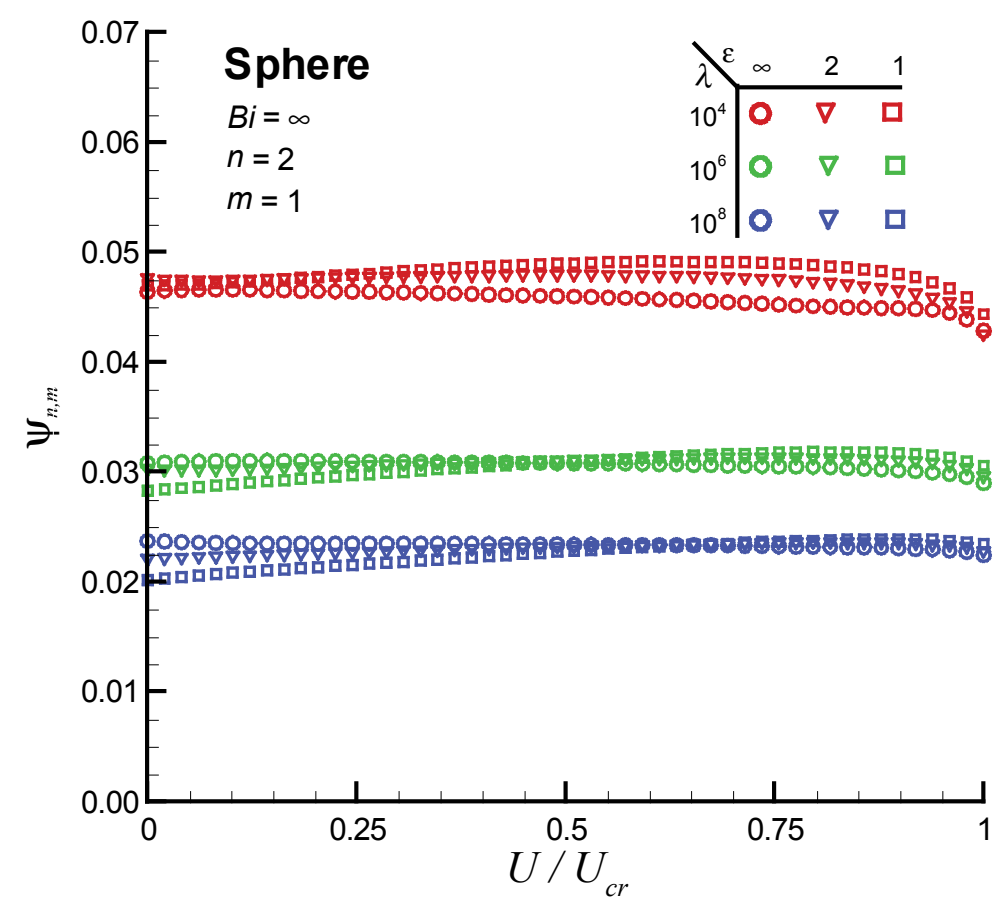

Figure 56. $\psi_{n, m}$ versus $U / U_{c r}$ for nonuniform spherical assemblies $(n=2, m=1)$.

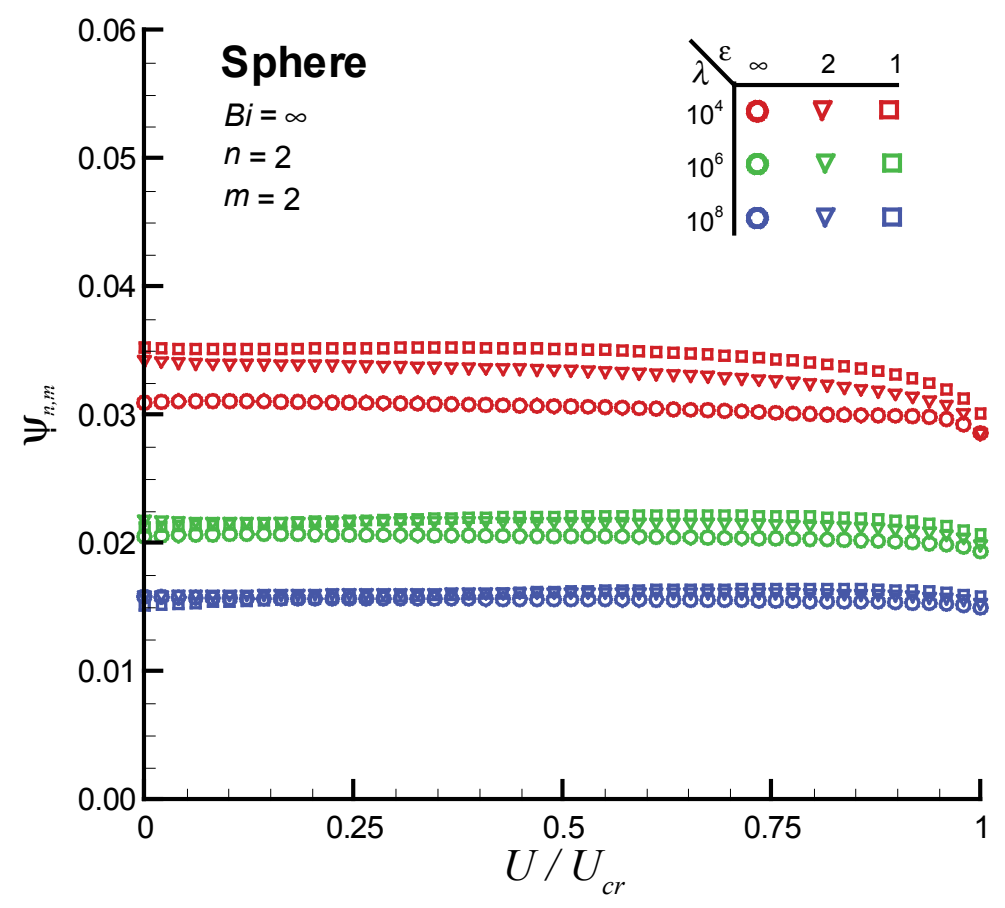

Figure 57. $\psi_{n, m}$ versus $U / U_{c r}$ for nonuniform spherical assemblies $(n=2, m=2)$. 
Table 6. Arithmetically averaged values of spatial moment integrals, $\bar{\psi}_{m, n}$.

\begin{tabular}{|c|c|c|c|}
\hline Slab $(\boldsymbol{n}=\mathbf{0})$ & $\boldsymbol{\lambda}=\mathbf{1 0}^{\mathbf{4}}$ & $\boldsymbol{\lambda}=\mathbf{1 0}^{\mathbf{6}}$ & $\boldsymbol{\lambda}=\mathbf{1 0}^{\mathbf{8}}$ \\
\hline$m=1$ & 0.041 & 0.028 & 0.0022 \\
$m=2$ & 0.028 & 0.019 & 0.015 \\
\hline
\end{tabular}

\begin{tabular}{|c|c|c|c|}
\hline \hline Cylinder $(\boldsymbol{n}=\mathbf{1})$ & $\boldsymbol{\lambda}=\mathbf{1 0}^{\mathbf{4}}$ & $\boldsymbol{\lambda}=\mathbf{1 0}^{\mathbf{6}}$ & $\boldsymbol{\lambda}=\mathbf{1 0}^{\mathbf{8}}$ \\
\hline$m=1$ & 0.045 & 0.030 & 0.022 \\
$m=2$ & 0.031 & 0.021 & 0.015 \\
\hline \hline Sphere $(\boldsymbol{n}=\mathbf{2})$ & $\boldsymbol{\lambda}=\mathbf{1 0}^{\mathbf{4}}$ & $\boldsymbol{\lambda}=\mathbf{1 0}^{\mathbf{6}}$ & $\boldsymbol{\lambda}=\mathbf{1 0}^{\mathbf{8}}$ \\
\hline$m=1$ & 0.047 & 0.031 & 0.023 \\
$m=2$ & 0.033 & 0.021 & 0.016 \\
\hline
\end{tabular}

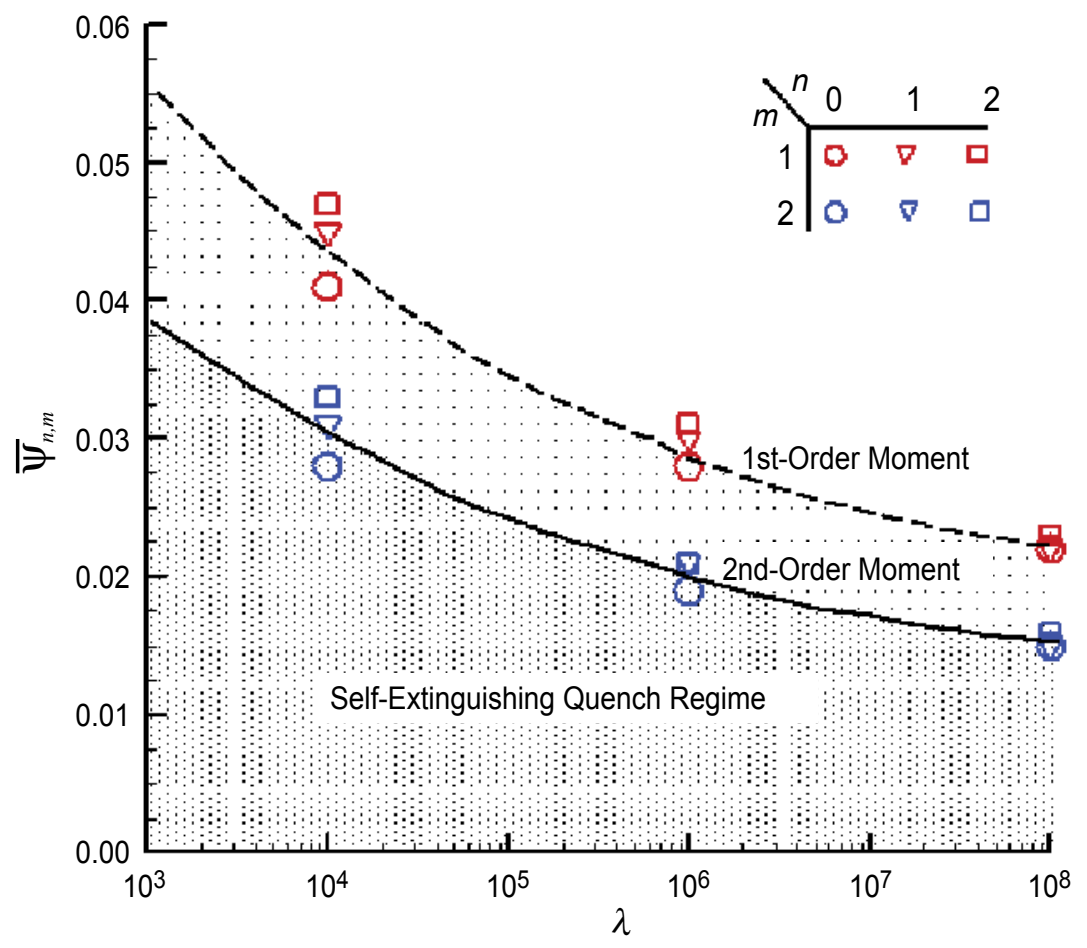

Figure 58. Arithmetically averaged values of spatial moment integrals, $\bar{\psi}_{m, n}$. 


\section{DYNAMIC BOUNDARY CONDITIONS}

Frequently, self-heating assemblies of hazardous materials are inadvertently exposed to time-varying ambient temperature conditions that can affect the critical threshold for thermal ignition/explosion. This might occur, for instance, if the material were stored in a location having inadequate climate control provisions and the diurnal temperature variations happened to be sufficiently large. It could also occur during the transport of a holding container if it were to be haphazardly placed near a fluctuating heat source. How a particular assembly would respond to such dynamic regimes of ignition is therefore a question of great practical importance.

Physical situations involving dynamic boundary conditions, where the ambient temperature or surface heat flux has a known time dependent variation, represent a class of problems that require a formal nonstationary mathematical treatment. The standard stationary model for static regimes of ignition is not directly applicable to this situation, and no viable construct has ever been found that would allow its use for approximation purposes. In the most general case, the transient solution may be expected to display a marked sensitivity to ambient temperature fluctuations, and we may anticipate that this will have a significant impact on the threshold of criticality. The objective of this chapter is to examine the effects of dynamic boundary conditions on the classic 'storage' problem and to use the results of the nonstationary model to lay the foundations for the development of an approximate solution methodology based on adaptation of the standard stationary model. For clarity, this investigation will only consider the exposure of simple centrosymmetric geometries to periodic oscillations in ambient temperature.

\subsection{Nonstationary Model Development}

Recall that $U$ was treated as a time dependent variable in the numerical development of the nonstationary model, which allows the surface boundary condition to be arbitrarily specified as a dynamic external driving force. Therefore, $U^{m}$ and $U^{m+1}$ are directly specified in equation $(72, i=N)$ at each time step according to some predefined temporal variation in the ambient environment. Although this dynamic variation can, in principle, obey any conceivable mathematical construct, the most frequently encountered situation of practical physical significance involves oscillatory patterns associated with diurnal variations in temperature. Here, we shall confine our attention to the classic storage problem assuming an infinite Biot number and time-varying ambient temperature. To limit the scope of study, we restrict attention to the uniform initial temperature distribution only.

\subsubsection{Sinusoidal Ambient Temperature Oscillation}

The simplest construct representing periodic oscillation is the sinusoidal harmonic oscillator with arbitrary amplitude and period. For our purposes, this is a convenient and useful representation for investigating the influence of an oscillatory surface boundary condition, and we adopt the following simple form for the instantaneous ambient temperature: 


$$
U=\bar{U}+U^{\prime} \sin (2 \pi \tau / T+\varphi),
$$

where $\bar{U}$ is the time-averaged mean value, $U^{\prime}$ is the fluctuation amplitude, $T$ is the period of oscillation, and $\varphi$ is the phase angle. Our principal interest is to examine the behavior of the critical ignition threshold under the influence of this dynamic boundary condition over a limited range of parameter values.

\subsubsection{Provisional Verification}

Previously, in section 3.2, we examined representative ignition threshold calculations assuming an initial uniform temperature distribution within the reactive material and constant ambient temperature. Given values for $\lambda, U$, and $B i$, our principal interest was to determine the critical value, $C_{c r}$, and verify nonstationary model performance. Here, we extend these calculations to include dynamic boundary condition effects and present provisional verification results.

The previous validation calculations were performed for all three principal centrosymmetric solids using Crank-Nicholson time integration $(f=0.5)$ with the controlling physical parameters defined as $\lambda=10^{6}, \bar{U}=0.05$, and $B i \rightarrow \infty$. Critical initial threshold temperatures were determined through trial and error, and representative transients for the dimensionless centerline temperature were shown in figures 13 , 14 , and 15 for $n=0, n=1$, and $n=2$, respectively.

In this section, we revisit calculations for the $n=0$ and $n=2$ cases under the action of a sinusoidal oscillating ambient boundary condition where a very small ambient temperature fluctuation $\left(U^{\prime}=0.001\right)$ is imposed on the mean ambient temperature $(\bar{U}=0.05)$. The period of oscillation was $T=0.1$, a value which is substantially less than the characteristic ignition induction time, $\tau_{\text {ind }}$. For simplicity, we have assumed $\varphi=0$.

These results are summarized in figures 59 and 60 for the planar and spherical geometries, respectively. Note that the critical ignition threshold values for the oscillating ambient temperature cases are less than those obtained using fixed ambient temperature conditions. In the planar slab, for instance, we observe that $C_{c r}=0.0042$ for the dynamic boundary condition compared to $C_{c r}=0.0043$ for the static boundary condition. Similarly for the spherical geometry, we find that $C_{c r}=0.0100$ for the dynamic boundary condition in comparison to $C_{c r}=0.0110$ for the static boundary condition. The significant conclusion appears to be that fluctuating dynamic boundary conditions generally act to reduce the ignition threshold below that associated with the corresponding static environment.

This conclusion has important practical consequences for the classic storage problem when the environment is known to exhibit nonnegligible temporal variations in temperature. Moreover, the principal effect of these fluctuations is to lower the ignition threshold and increase the risk for an accidental fire. This risk is further accentuated when the stationary model is blindly applied to the storage problem under environmental conditions known to have inherent dynamical variation. The purpose of the current study is to raise awareness of these oft neglected considerations and point the way toward the development of practical predictive capabilities. 


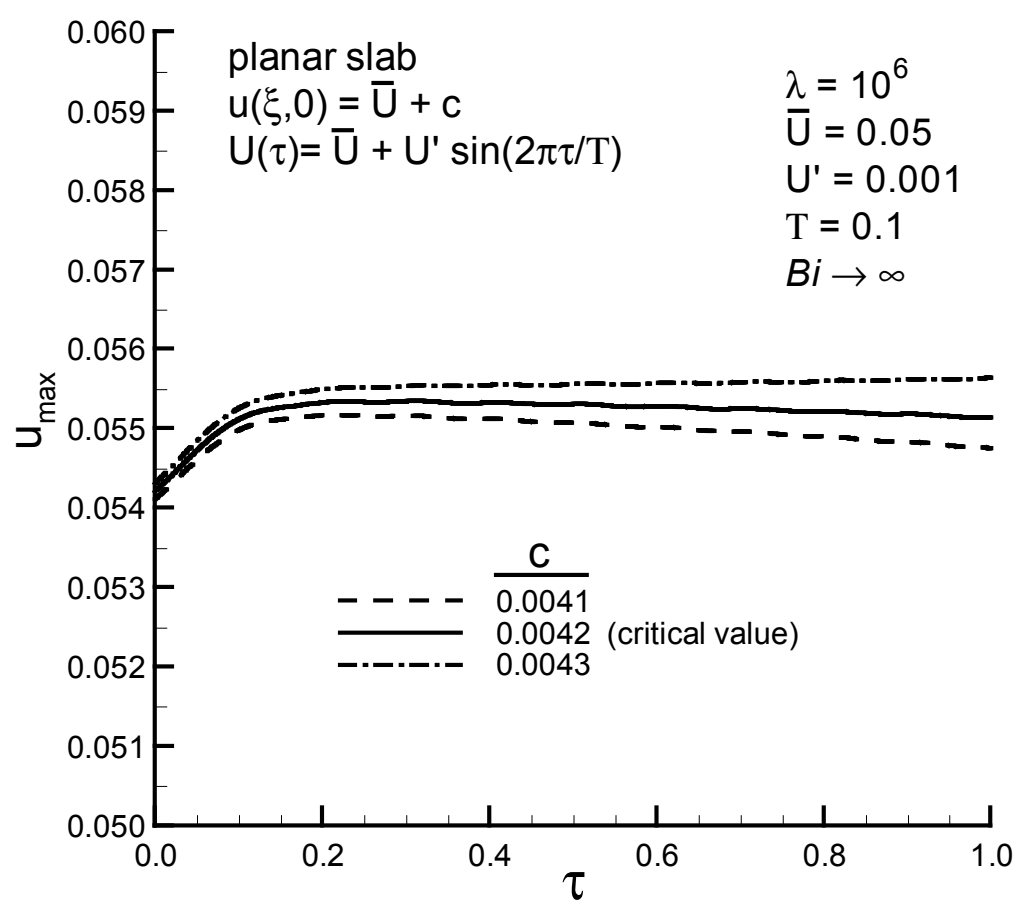

Figure 59. Centerline temperature transients for $n=0\left(\lambda=10^{6}\right.$ and $\left.B i \rightarrow \infty\right)$ with sinusoidal oscillating ambient temperature $\left(\bar{U}=0.05, U^{\prime}=0.001\right.$, and $\left.T=0.1\right)$.

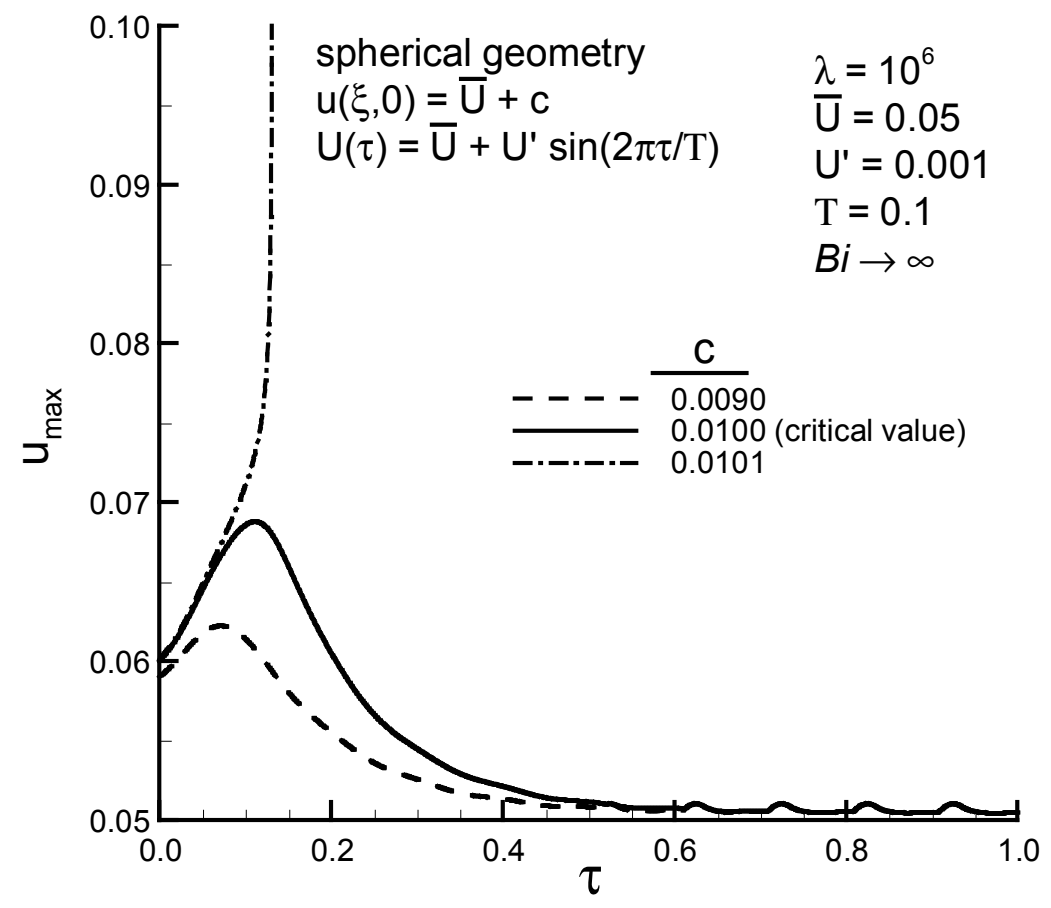

Figure 60. Centerline temperature transients for $n=2\left(\lambda=10^{6}\right.$ and $\left.B i \rightarrow \infty\right)$ with sinusoidal oscillating ambient temperature $\left(\bar{U}=0.05, U^{\prime}=0.001\right.$, and $\left.T=0.1\right)$. 


\subsection{Parametric Survey}

We now extend the scope of our dynamic boundary condition survey and examine ignition characteristics over a wider range of parameter values. To avoid undue clutter at the expense of minor additional insight gained from completeness, we restrict attention to the planar slab geometry $(n=0)$ assuming an initial uniform temperature distribution and impose the sinusoidal ambient temperature oscillation defined by equation (102). Again, the controlling physical parameters are taken to be $\lambda=10^{6}, \bar{U}=0.05$, and $B i \rightarrow \infty$.

Before proceeding with detailed calculations of the critical ignition threshold, we first use the nonstationary model to compute ignition induction times, as measured from the critical initial temperature $u_{c r}$, for the fixed boundary state. The results are plotted in figure 61, which shows the variation in $\tau_{\text {ind }}$ as a function of $U$. If the time to ignition is computed from some fixed initial temperature, we would expect that $\tau_{\text {ind }} \propto \exp [1 / U]$ (ref. 1, p. 208). However, when the time to ignition is based on the critical initial threshold temperature corresponding to the value of $U$, we find that the induction time is relatively small when $U$ is much less than $U_{c r}$ and grows slowly until $U$ approaches $U_{c r}$, at which point it experiences explosive growth in magnitude. When $U<<U_{c r}, \tau_{i n d} \approx 0.1-0.3$, but as $U \rightarrow U_{c r}$, we observe that $\tau_{\text {ind }} \rightarrow 0.1$ very rapidly in the neighborhood of $U_{c r}$. This result will appear counter-intuitive unless one keeps in mind the fact that the induction time defined here is based on the critical initial temperature, $u_{c r}$, which decreases as $U$ increases.

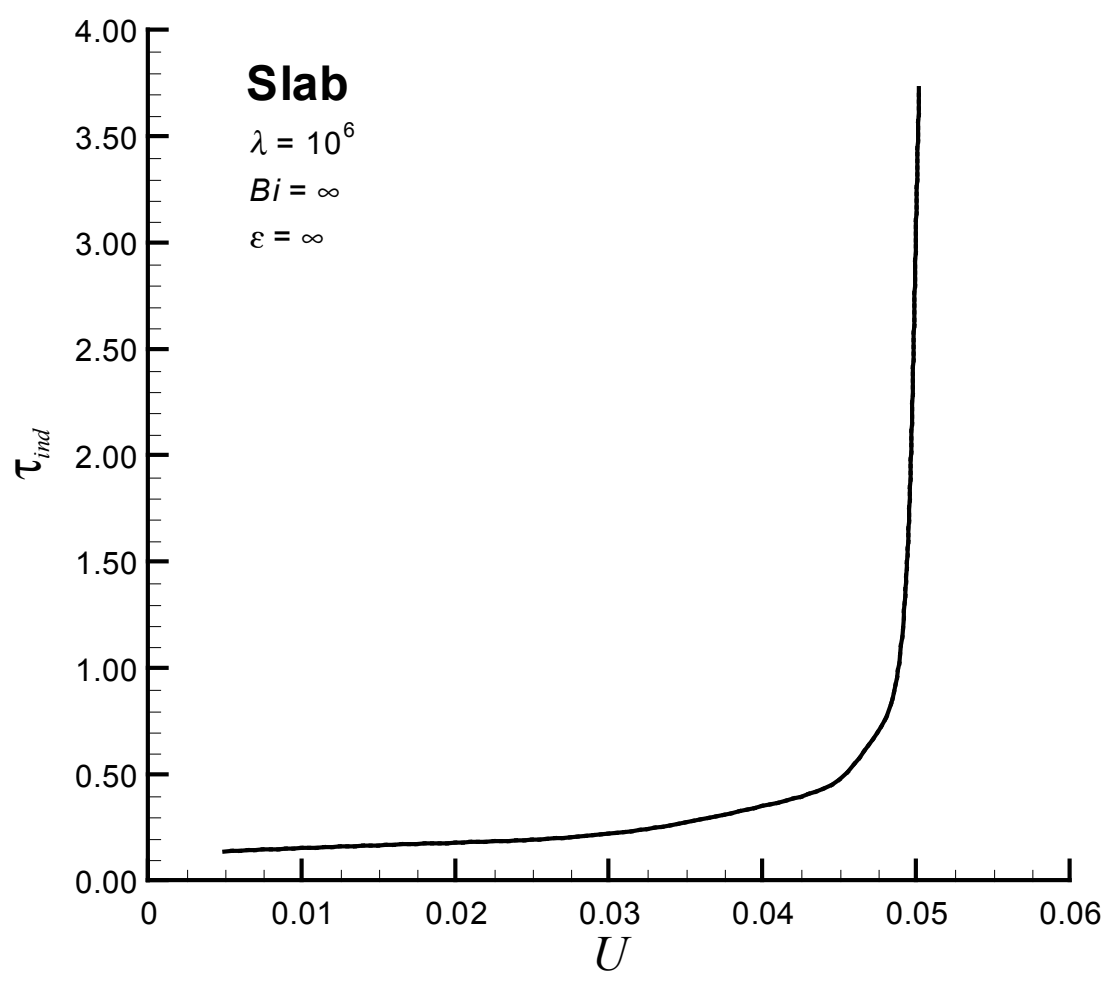

Figure 61. Variation in $\tau_{\text {ind }}$ as a function of $U$ for $n=0\left(\lambda=10^{6}\right.$ and $\left.B i \rightarrow \infty\right)$. 
Based on the preceding observations, we intuitively reason that periods of oscillation longer than the ignition induction time should have minimal effects on the predicted critical threshold conditions. Therefore, the primary range of interest for the period of oscillation extends over values less than or equal to $\tau_{i n d}$. In order to establish representative test cases, two specific values for the period of oscillation were chosen for detailed investigation. These included a baseline case where $T$ is on the order of the minimum value of $\tau_{\text {ind }}$ (i.e., $T=0.1$ ) and an additional special case where $T$ is significantly less than $\tau_{\text {ind }}$ (i.e., $T=0.05$ ). We do not examine any effects associated with variable phase angle and simply take $\varphi=0$ to satisfy the limited purpose of this study.

Nonstationary model calculations were then undertaken using the selected values for the period of oscillation with fluctuation amplitudes ranging from 0 to 0.025 . The results of these survey calculations are summarized in figures 62 and 63, which depict the critical threshold curves $u(0)=U+C_{c r} g(0)$ versus $\bar{U}$ as well as the stationary model bifurcation curve corresponding to the fixed ambient temperature condition.

There are several noteworthy points of observation that may be derived from these graphs. Most significantly, it is evident that the oscillating ambient temperature acts to substantially reduce the critical ignition threshold across the entire range of mean ambient temperatures. This reduction becomes gradually more pronounced as the fluctuation amplitude increases. Furthermore, the degree of reduction is found to be highly magnified when $\bar{U}$ is small and the ignition induction time is relatively short. As $\bar{U}$ increases, however, the critical threshold curves are found to undergo a strong nonlinearity and are observed to exhibit an almost discontinuous jump if $U^{\prime}$ is sufficiently large. From a practical perspective, it is important to note that $U_{c r}$ decreases with increasing $U^{\prime}$, although the magnitude of this reduction does not appear to be strongly affected by changes in the period of oscillation over the parameter range of the study.

\subsection{Correlation}

From an applied mathematics perspective, the important questions are associated with how dynamic boundary conditions in the assembly problem affect the critical ambient temperature and whether any insight may be derived from this behavior that might provide a useful predictive capability for hazard analysis and risk assessment purposes. The parameter space under the action of dynamic boundary conditions is immense and essentially intractable from an analytical perspective, but some constructive progress may be expected from an attempt at correlation of numerical results. Let us begin by examining how the critical ambient temperature varies with fluctuation amplitude and period of oscillation for the special test case under consideration.

Variation in $U_{c r}$ with $U^{\prime}$ and $T$ is summarized in table 7. These results are also depicted graphically in figure 64. Note that $U_{c r}$ declines as the magnitude of $U^{\prime}$ increases and that the reduction effect becomes less pronounced as the period is decreased from $T=0.1$ to $T=0.05$. The greatest effect occurs for large values of $U^{\prime}$, as would be intuitively expected. Apparently, the longer period makes the system more prone to ignition by allowing the upper half cycle to exert more influence in comparison to the lower half cycle. That is, criticality seems to be affected more by prolonged heat loss suppression during the upper half cycle than by prolonged heat loss enhancement during the lower half cycle. This result should not be blindly accepted as a definitive deduction, however, since it is impossible to draw general conclusions 


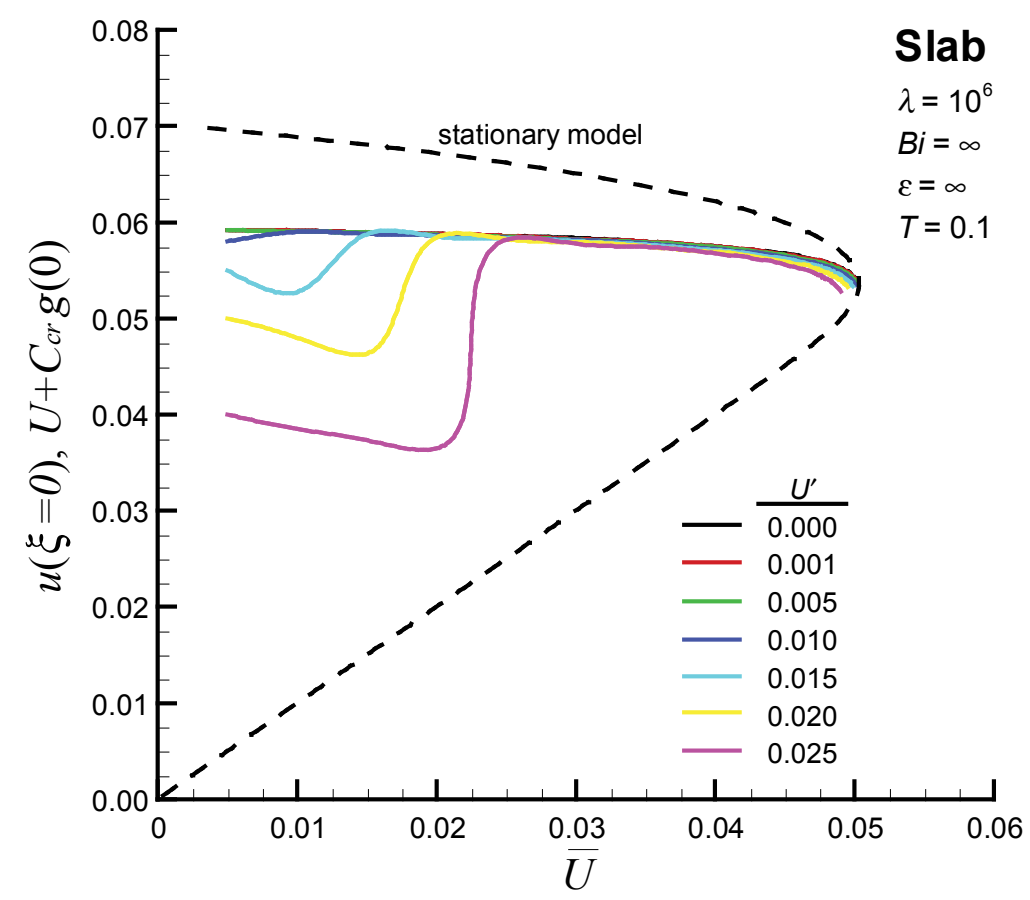

Figure 62. Criticality characteristics $\left(n=0, \lambda=10^{6}, \bar{U}=0.05\right.$, and $\left.B i \rightarrow \infty\right)$ with sinusoidal oscillating ambient temperature $(T=0.1)$.

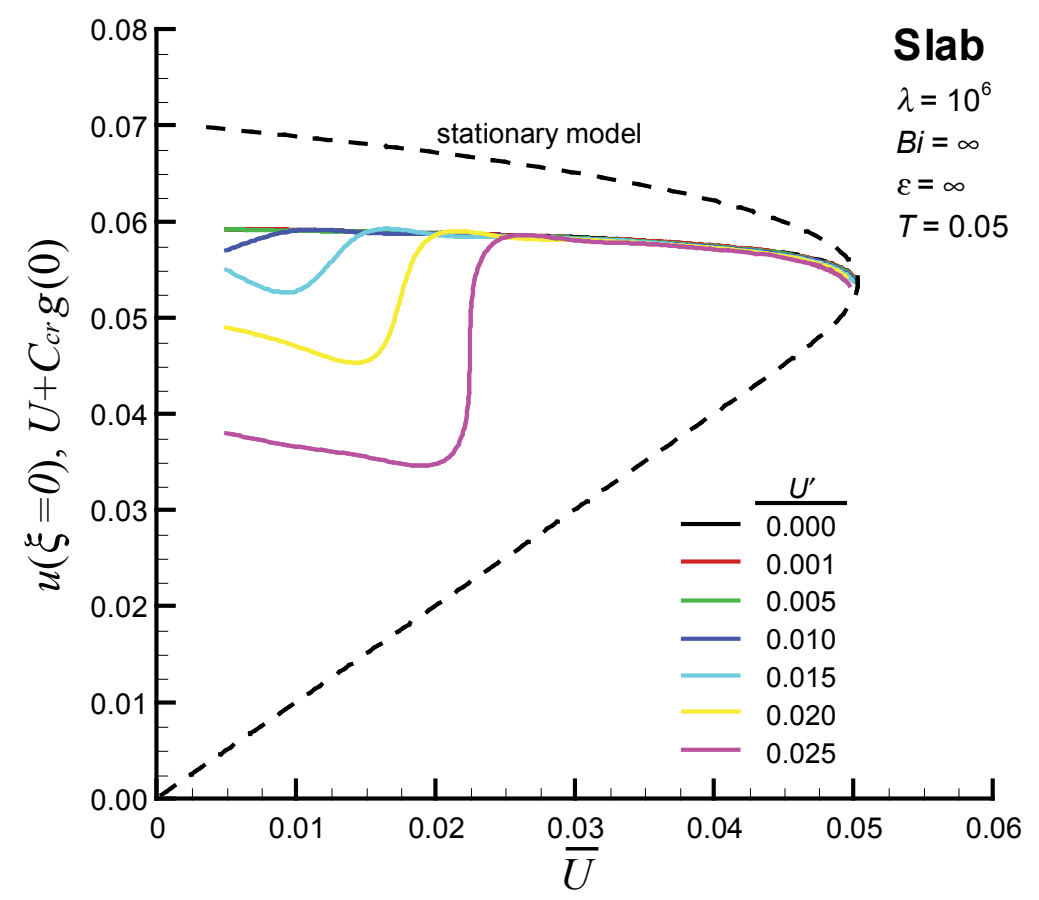

Figure 63. Criticality characteristics $\left(n=0, \lambda=10^{6}, U=0.05\right.$, and $\left.B i \rightarrow \infty\right)$ with sinusoidal oscillating ambient temperature $(T=0.1)$. 
Table 7. Variation in $U_{c r}$ with $U^{\prime}$ and $T$.

\begin{tabular}{|l|c|c|}
\hline \multicolumn{1}{|c|}{$\boldsymbol{U}^{\prime}$} & $\boldsymbol{U}_{\boldsymbol{c r}}(\boldsymbol{T}=\mathbf{0 . 1})$ & $\boldsymbol{U}_{\boldsymbol{c r}}(\boldsymbol{T}=\mathbf{0 . 0 5})$ \\
\hline 0 & 0.05033 & 0.05033 \\
0.001 & 0.05028 & 0.05028 \\
0.005 & 0.05022 & 0.05022 \\
0.010 & 0.05010 & 0.05019 \\
0.015 & 0.04991 & 0.05006 \\
0.020 & 0.04951 & 0.04991 \\
0.025 & 0.04903 & 0.04971 \\
\hline
\end{tabular}

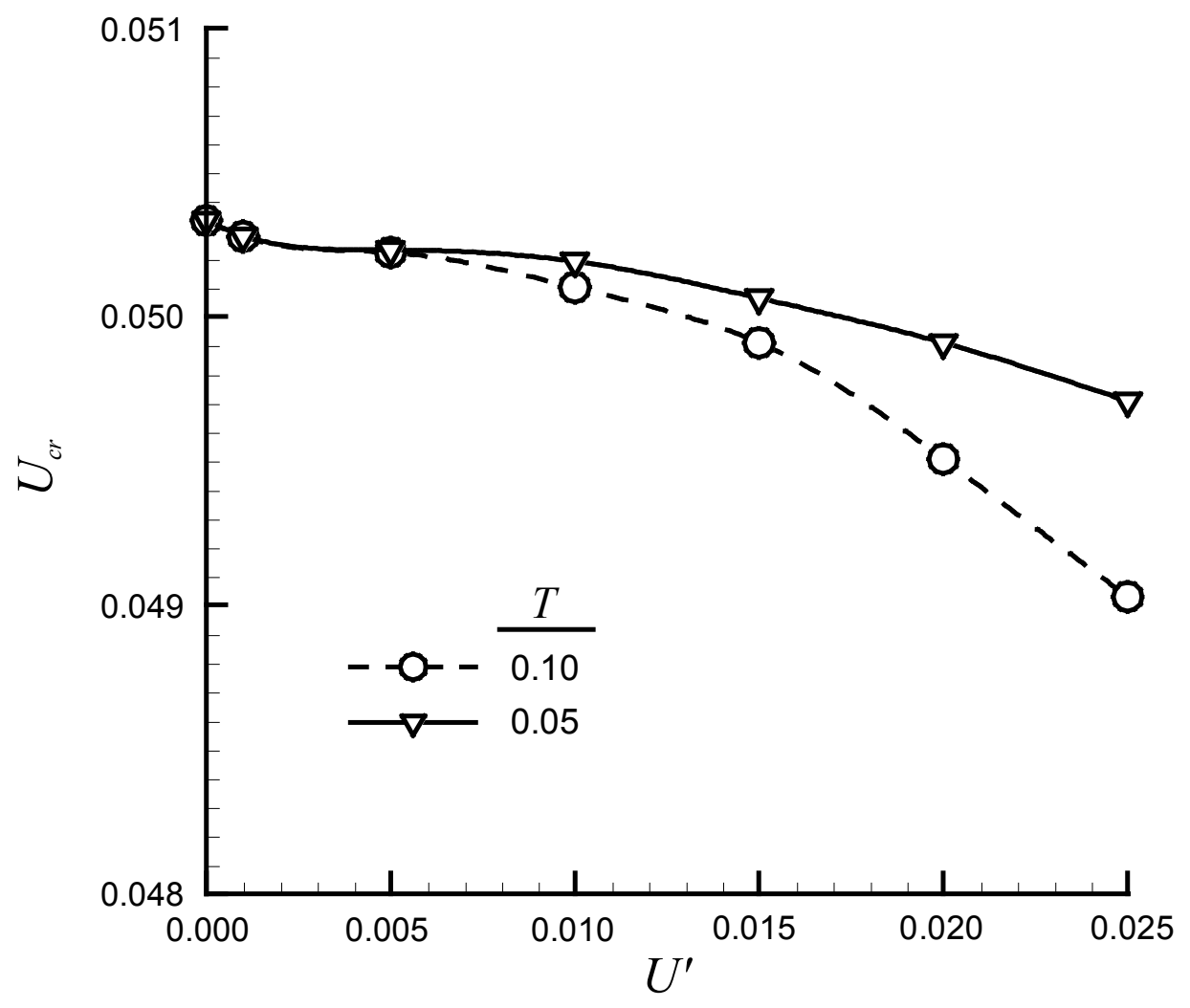

Figure 64. Variation in $U_{c r}$ with $U^{\prime}$ and $T$ for $n=0\left(\lambda=10^{6}\right.$ and $\left.B i \rightarrow \infty\right)$ with sinusoidal oscillating ambient temperature.

from this preliminary and cursory analysis. Ultimately, more in-depth analysis and study will be needed to fully resolve such issues to reach reliable deductions.

The question that needs to be addressed is how such results can be used for predictive purposes. Is there some way, for instance, that the stationary model can be modified and effectively utilized to generate useful predictions? Here, we suggest one simple approach based on the introduction of an effective Biot 
number to represent the effect of oscillating ambient temperature. To do so, $B i$ is varied in the stationary model in order to obtain a value for $U_{c r}$ which is in agreement with the tabulated values of table 7 and thereby deduce an effective Biot number, $B i_{\text {eff }}$. The results of this correlating exercise are summarized in the graph of figure 65, which depicts $B i_{e f f}$ as a function of $U^{\prime}$ for both oscillation periods of interest. The accuracy of this correlation is quite good and opens the door to a potentially simple predictive methodology for the dynamic boundary storage problem. A critical assessment of feasibility and comprehensive development of the approach is beyond the current scope of study and is left for future examination.

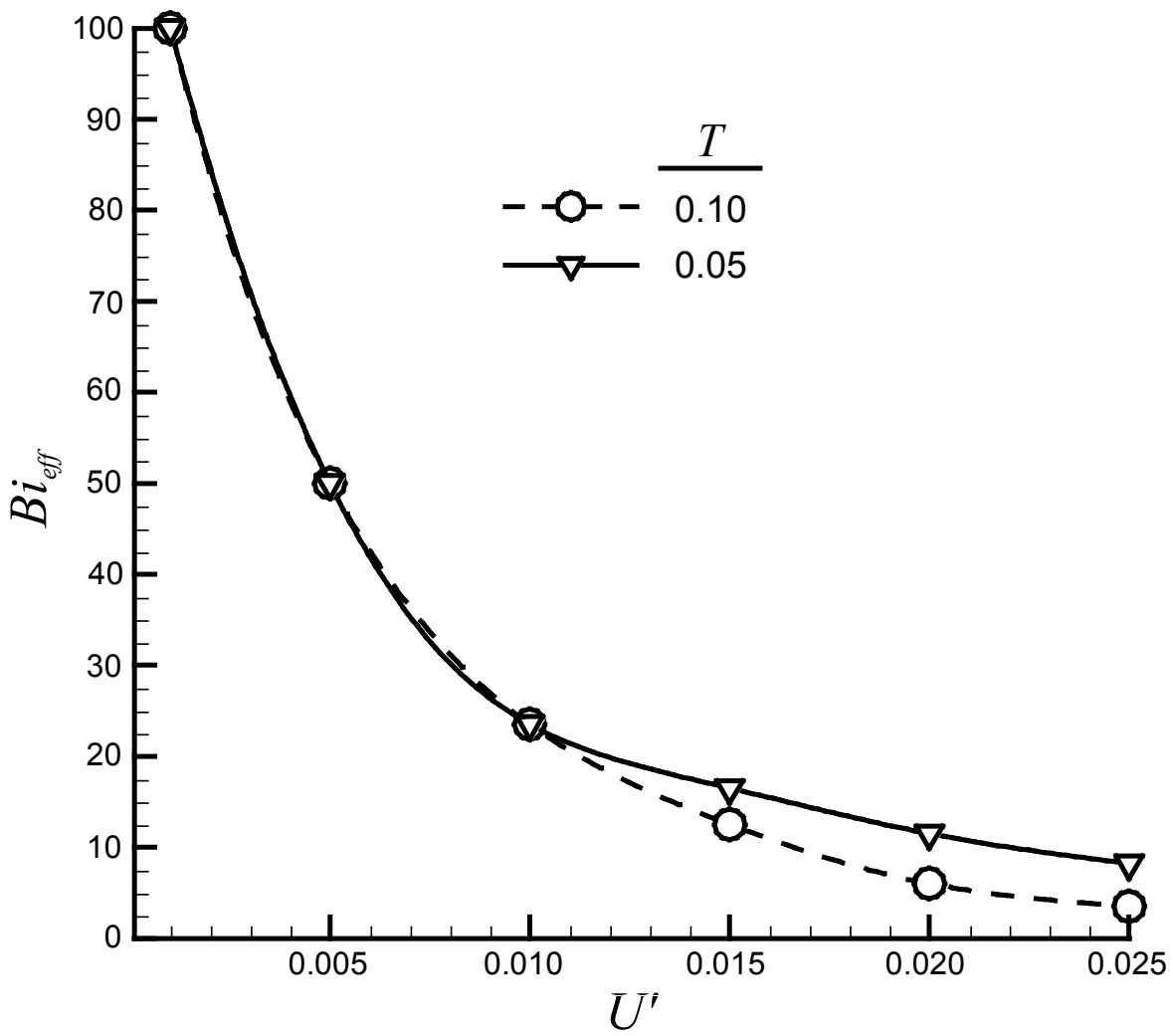

Figure 65. Stationary model correlation for $B i_{\text {eff }}$ as a function of $U^{\prime}$ and $T$ for $n=0$ $\left(\lambda=10^{6}\right.$ and $\left.B i \rightarrow \infty\right)$ with sinusoidal oscillating ambient temperature. 


\section{CONCLUDING REMARKS}

Prior to this work, a number of practically important questions remained unanswered concerning the critical threshold for thermal ignition, given an assembly problem with a nonuniform initial temperature distribution. To seek answers to such questions, the reaction-diffusion equation in the dimensionless form $\partial_{t} u=\nabla^{2} u+\lambda e^{-1 / u}$ over the bounded region $\Omega$ of the principal centrosymmetric solids was critically reexamined from the perspective of modern numerical methodologies. The purpose was to broadly and deeply penetrate the solution space in order to reveal a more detailed description of the underlying mathematical structure, which could be further used to establish conjectural and correlating principles of general predictive utility.

As part of this undertaking, the classic stationary model was first revisited, and an innovative solution methodology was developed whereby the two-point boundary value problem could be reexpressed as an initial value problem for a system of first-order ordinary differential equations. Careful validation calculations demonstrate that this simplified approach was capable of yielding bifurcation diagrams just as accurate as more sophisticated path following techniques.

A numerical procedure was then implemented for solving the full time-dependent form of the reaction diffusion equation and establishing a nonstationary thermal ignition model. This numerical development combined second-order central differencing for the spatial derivative with a generalized time integration scheme, including a time-dependent convective boundary condition, to produce an efficient and accurate solver routine. The resulting methodology was successfully validated against published solutions in the peer-reviewed literature and found robust and reliable.

Having developed and validated stationary and nonstationary models, a judicious examination of the assembly problem was undertaken to expose how the fate of a self-heating material is dependent on the internal spatial concentration of thermal energy at the time of assembly. By introducing a normalized shape profile for the initial temperature distribution, as defined by a single geometric parameter, it was then possible to compute criticality threshold characteristics over a broad range of practical values for the dimensionless eigenvalue parameter.

It was shown how the resulting mathematical structure for the ignition threshold curves could be correlated by a hyperbolic conic section with a high degree of fidelity over the full range of positive ambient temperature values. Moreover, the clever introduction of new dimensionless parameters within this hyperbolic correlating form was found to generate further simplification leading to a universal correlating form capable of collapsing the entire solution space onto a single line in the plane of the new variables. This result was found to hold over a wide range of shape parameters and is therefore believed to be of general significance.

One of the major open questions addressed by this study of the assembly problem concerned the widely held conviction that certain spatial moments of the initial temperature profile ought to possess a 
direct mathematical link to the critical ignition threshold. As such, the $m$ th order spatial moment of the critical total energy content integrals was defined, and a conjecture was formulated stating that certain orders of this moment would remain invariant with changes in ambient temperature and initial shape profile and would therefore be functionally dependent on the dimensionless eigenvalue only, within some quantifiable error band. Evaluations of the spatial moment integrals demonstrated this conjecture to be provisionally valid, with the best accuracy obtained for second order moments. The invariance property for the spatial moment turns out to be quite powerful and yields a simple but fairly accurate method for estimating fire hazard risks for thermally nonuniform assemblies of self-heating materials. The framing and verification of this conjecture represents a significant accomplishment and greatly expands our mathematical understanding of this practically important problem.

As a final focal point for this work, an attempt was made to undertake a preliminary and cursory analysis of the classic storage problem with dynamic boundary conditions assuming infinite Biot number. Specifically, an examination was made of ignition characteristic for the planar slab geometry with initially uniform temperature and sinusoidal oscillating ambient temperature. The critical threshold limits were computed assuming a range of fluctuation amplitudes for two representative periods of oscillation. It was found that an oscillating boundary condition generally acts to reduce the critical ignition threshold with the degree of reduction increasing with increasing oscillation amplitude. It was also shown how the stationary model could be used to correlate variations in the critical ambient temperature with changes in the fluctuation amplitude by defining an effective Biot number to represent a virtual heat loss suppression mechanism. That is, there is an equivalent finite effective Biot number for which the stationary model accurately predicts the critical ambient temperature corresponding to the dynamic boundary storage problem with infinite Biot number. It should be emphasized that this cursory result, based on a limited exploration of an immense solution space, must be viewed as preliminary in nature and not a statement of general validity. However, it is believed that this result is highly suggestive of a potentially promising approach to an otherwise intractable problem and is deserving of further study and investigation. 


\section{REFERENCES}

1. Bowes, P.C.; Self Heating: Evaluating and Controlling the Hazards. Elsevier Science Publishing Company, New York, 1984.

2. Rivers, C.M.; Wake, G.C.; and Chen, X.D.: Math. Eng. Ind., Vol. 6(1), 1996.

3. Smedley, S.I.; and Wake, G.C.: "Spontaneous Ignition: Assessment of Cause." Annual Meeting of the Institute of Loss Adjusters of NZ, Palmerston North, NZ, June 1987.

4. Weber, E.R.; Balakrishnan, O.; and Wake, G.C.: "Critical Initial Conditions for Spontaneous Thermal Ignition,” J. Chem. Soc., Faraday Trans., Vol. 94, pp. 3613-3617, 1998.

5. Frank-Kamanetskii, D.A.: Diffusion and Heat Transfer in Chemical Kinetics, translated by J.P. Appleton. Plenum Press, Princeton, New York, 1969.

6. Burnell, J.G.; Graham-Eagle, J.G.; Gray, B.F.; and Wake, G. C.: "Determination of Critical Ambient Temperatures for Thermal Ignition.” IMA Jour. Appl. Math., Vol. 42(147) p.154, 1989.

7. Boddington, T.; Gray, P.; and Wake, G.C.: "Criteria for Thermal Explosion With and Without Reactant Consumption,” Proc. R. Soc. London A., Vol. 357, pp. 403-422 (1977).

8. Boddington, T.; Gray, P.; and Harvey, D.I.: "Thermal Theory of Spontaneous Ignition: Criticality in Bodies of Arbitrary Shape.” Philos. Trans. Royal Soc. London, Vol. A270, pp. 467-506, 1971.

9. Wake, G.C.; and Walker, I.K.: "Calorimetry of Oxidation Reactions." N.Z. Jl. Sci. , Vol 7 (2), p. $227,1964$.

10. Balakrishnan, E.: Numerical Study of Thermal Ignition in the New Variables, Ph.D. Thesis, Massey University, New Zealand, 1995.

11. Amann, H.: "Fixed Point Equations and Nonlinear Eigenvalue Problems in Ordered Banach Spaces," SIAM Rev, Vol.18, pp. 620-709, 1976.

12. Aris, K.: The Mathematical Theory of Diffusion and Reaction in Permeable Catalysts, Vols. 1 and 2, Oxford University Press, 1975.

13. Wake, G.C; Gray, B.F.; Graham-Eagle, J.G.; and Burnell, J.B: "A New Scaling of a Problem in Combustion Theory," in Reaction-Diffusion Equations, pp. 25-38, Oxford Science, 1990.

14. Gray, B.F.; Wake, G.C.; and Merkin, J.H.: "Disjoint Bifurcation Diagrams in Combustion Systems," Mathl. Comput. Modelling, Vol. 18 (8), pp. 65-75, 1993. 
15. Keller, H.B: "Numerical Solutions of Bifurcation and Nonlinear Eigenvalue Problems," in Applications of Bifurcation Theory, Academic Press, 1977.

16. Iooss, G.; and Joseph, D.D.: Elementary Stability and Bifurcation Theory, Academic Press, 1977.

17. Chow, S.N.; and Hale, J.K.: Methods of Bifurcation Theory, Springer-Verlag, New York, 1982.

18. Rheinboldt, W.: Numerical Analysis of Parameterized Equations, John Wiley and Sons, New York, 1986.

19. Seydel, R.: From Equilibrium to Chaos: Practical Bifurcation and Stability Analysis, American Elsevier, New York, 1988.

20. Scotter, M.D.: "Path Following Methods for Boundary Value Problems and Their Applications to Combustion Equations," Ph.D. Thesis, Department of Mathematics, Massey University, New Zealand, 1991.

21. Allgower, E.L.; and Georg, K.: Numerical Continuation Methods: An Introduction, Springer-Verlag, New York, 1990.

22. Allgower, E. L.; and Georg, K.: "Continuation and Path Following," Acta Numerica, pp. 1-64, 1993.

23. Doedel, E.: AUTO: Software for Continuation and Bifurcation Problems in Ordinary Differential Equations, California Institute of Technology, 1986.

24. Gray, P.; and Harper, M.J.: In Seventh Symposium (International) on Combustion, p. 425, Butterworths, London, 1959.

25 Gray, P.; and Harper, M.J.: Trans Faraday Soc., Vol. 55, p. 581, 1959.

26. Hermance, C.E.: AIAA J., Vol. 11, p. 1728, 1973.

27. Kassoy, D.R.: "Homogeneous Explosions-Initiation to Completion." AIAA 12th Aerospace Sciences Meeting, Washington, DC, Paper AIAA-74-150, 1974.

28. Kassoy, D.R.: Combustion Science Technology, Vol.10, p. 27, 1975.

29. Kassoy, D.R.; and Poland, J.: Combustion Science Technology. Vol.11., p. 174, 1975.

30. Patankar, S.V.: Numerical Heat Transfer and Fluid Flow, Hemisphere Publishing, New York, 1980.

31. Rivers, C.M.; Wake, G.C.; and Chen, X.D.: Math. Eng. Ind., Vol. 6, page 1, 1996. 


\begin{tabular}{|c|c|c|c|}
\hline \multicolumn{3}{|c|}{ REPORT DOCUMENTATION PAGE } & $\begin{array}{l}\text { Form Approved } \\
\text { OMB No. 0704-0188 }\end{array}$ \\
\hline \multicolumn{4}{|c|}{ 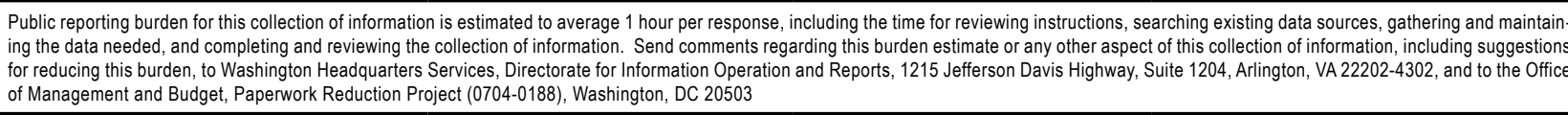 } \\
\hline 1. AGENCY USE ONLY (Leave Blank) & $\begin{array}{l}\text { 2. REPORT DATE } \\
\text { February } 2008\end{array}$ & \multicolumn{2}{|c|}{\begin{tabular}{|l} 
3. REPORT TYPE AND DATES COVERED \\
Technical Publication
\end{tabular}} \\
\hline \multicolumn{3}{|c|}{$\begin{array}{l}\text { 4. TITLE AND SUBTITLE } \\
\text { Numerical Determination of Critical Conditions for Thermal } \\
\text { Ignition }\end{array}$} & \multirow[t]{2}{*}{ 5. FUNDING NUMBERS } \\
\hline \multicolumn{3}{|c|}{$\begin{array}{l}\text { 6. Authors } \\
\text { W.Luo, }{ }^{*} \text { G.C. Wake, }{ }^{*} \text { C.W. Hawk, }{ }^{* *} \text { and R.J. Litchford }\end{array}$} & \\
\hline \multicolumn{3}{|c|}{$\begin{array}{l}\text { 7. PERFORMING ORGANIZATION NAME(S) AND ADDRESS(ES) } \\
\text { George C. Marshall Space Flight Center } \\
\text { Marshall Space Flight Center, AL } 35812\end{array}$} & $\begin{array}{l}\text { 8. PERFORMING ORGANIZATION } \\
\text { REPORT NUMBER } \\
\text { M-1218 }\end{array}$ \\
\hline \multicolumn{3}{|c|}{$\begin{array}{l}\text { 9. SPONSORING/MONITORING AGENCY NAME(S) AND ADDRESS(ES) } \\
\text { National Aeronautics and Space Administration } \\
\text { Washington, DC 20546-0001 }\end{array}$} & $\begin{array}{l}\text { 10. SPONSORING/MONITORING } \\
\text { AGENCY REPORT NUMBER } \\
\text { NASA/TP_-2008-215194 }\end{array}$ \\
\hline \multicolumn{4}{|c|}{$\begin{array}{l}\text { 11. SUPPLEMENTARY NOTES } \\
\text { Prepared by the Propulsion Systems Department. Engineering Directorate. } \\
\text { *Massey University, Auckland, New Zealand; **The University of Alabama in Huntsville }\end{array}$} \\
\hline \multicolumn{3}{|c|}{$\begin{array}{l}\text { 12a. DISTRIBUTION/AVAILABILITY STATEMENT } \\
\text { Unclassified-Unlimited } \\
\text { Subject Category } 28 \\
\text { Availability: NASA CASI } 301-621-0390\end{array}$} & 12b. DISTRIBUTION CODE \\
\hline \multicolumn{4}{|c|}{$\begin{array}{l}\text { 13. ABSTRACT (Maximum 200 words) } \\
\text { The determination of ignition or thermal explosion in an oxidizing porous body of material, as described by a dimensionless } \\
\text { reaction-diffusion equation of the form } \partial t u=\nabla 2 u+\lambda e-1 / \mathrm{u} \text { over the bounded region } \Omega \text {, is critically reexamined from a modern } \\
\text { perspective using numerical methodologies. First, the classic stationary model is revisited to establish the proper reference frame } \\
\text { for the steady-state solution space, and it is demonstrated how the resulting nonlinear two-point boundary value problem can be } \\
\text { reexpressed as an initial value problem for a system of first-order differential equations, which may be readily solved using standard } \\
\text { algorithms. Then, the numerical procedure is implemented and thoroughly validated against previous computational results based } \\
\text { on sophisticated path-following techniques. Next, the transient nonstationary model is attacked, and the full nonlinear form of the } \\
\text { reaction-diffusion equation, including a generalized convective boundary condition, is discretized and expressed as a system of } \\
\text { linear algebraic equations. The numerical methodology is implemented as a computer algorithm, and validation computations are } \\
\text { carried out as a prelude to a broad-ranging evaluation of the assembly problem and identification of the watershed critical initial } \\
\text { temperature conditions for thermal ignition. This numerical methodology is then used as the basis for studying the relationship be- } \\
\text { tween the shape of the critical initial temperature distribution and the corresponding spatial moments of its energy content integral } \\
\text { and an attempt to forge a fundamental conjecture governing this relation. Finally, the effects of dynamic boundary conditions on the } \\
\text { classic 'storage' problem are investigated and the groundwork is laid for the development of an approximate solution methodology } \\
\text { based on adaptation of the standard stationary model. }\end{array}$} \\
\hline \multirow{2}{*}{\multicolumn{3}{|c|}{$\begin{array}{l}\text { 14. SUBJECT TERMS } \\
\text { ignition, thermal explosion, self-heating, spontaneous combustion. }\end{array}$}} & $\begin{array}{r}\text { 15. NUMBER OF PAGES } \\
94\end{array}$ \\
\hline & & & \begin{tabular}{|l} 
16. PRICE CODE \\
\end{tabular} \\
\hline $\begin{array}{l}\text { 17. SECURITY CLASSIFICATION } \\
\text { OF REPORT } \\
\text { Unclassified }\end{array}$ & $\begin{array}{l}\text { 18. SECURITY CLASSIFICATION } \\
\text { OF THIS PAGE } \\
\text { Unclassified }\end{array}$ & $\begin{array}{l}\text { 19. SECURITY CLASSIFICATION } \\
\text { OF ABSTRACT } \\
\text { Unclassified }\end{array}$ & $\begin{array}{l}\text { 20. LIMITATION OF ABSTRACT } \\
\text { Unlimited }\end{array}$ \\
\hline
\end{tabular}



National Aeronautics and

Space Administration

IS20

George C. Marshall Space Flight Center

Marshall Space Flight Center, Alabama

35812 